\title{
Bifurcação de Hopf para uma Família \\ a um Parâmetro de Equações Diferenciais com Retardamento no Plano
}

\section{Wania Cristina de Lucca}

\author{
Orientação \\ Prof. Dr. Plácido Zoéga Táboas
}

Dissertação apresentada ao Instituto de Ciências Matemáticas de São Carlos USP, como parte dos requisitos para obtenção do título de Mestre em Matemática.

\section{USP - São Carlos}

\author{
maio 1992
}


Inato no homem é o gosto de amassar, remexer e misturar.

Goethe 
Aos meus pais. 


\begin{abstract}
We study the Hopf Bifurcation Theorem for functional differential equations of retarded type. An application of this theorem is given for a planar differential difference equation.
\end{abstract}




\section{Resumo}

Estudamos o Teorema da Bifurcação de Hopf para equações diferenciais funcionais do tipo retardado. Uma aplicação deste teorema é dada para uma equação diferencial diferença no plano. 


\section{Conteúdo}

Introdução $\quad$ iii

1 Equações Diferenciais Funcionais Retardadas 1

1.1 Teoria Básica $\ldots \ldots \ldots \ldots \ldots \ldots \ldots \ldots$

1.2 Equações Lineares Autônomas com Retardamento . . . . . . . 4

1.3 Equação Linear Autônoma Forçada . . . . . . . . . 31

2 Bifurcação de Hopf $\quad 37$

2.1 Fatos Básicos da Teoria Geral . . . . . . . . . . . . 37

2.2 A aproximação linear e os auto valores $\ldots \ldots \ldots \ldots \ldots$

2.3 Teorema da Bifurcação de Hopf $\ldots \ldots \ldots \ldots$. . . . . . 41

3 Uma aplicação a equações diferenciais diferença no plano 54

3.1 Considerações Iniciais . . . . . . . . . . . . . . 54

3.2 A equação característica $\ldots \ldots \ldots \ldots \ldots \ldots \ldots$

3.3 Os auto valores da equação linearizada $\ldots \ldots \ldots \ldots \ldots 6$

A Fórmula da Variação das Constantes 65

$\begin{array}{ll}\text { B Método de Liapunov - Schmidt } & 70\end{array}$ 
Bibliografia 


\section{Introdução}

O comportamento futuro de muitos fenômenos físicos pode ser descrito pelas soluções de uma equação diferencial ordinária. Neste caso, assume-se que, estabelecido um instante inicial, esse comportamento independe do passado, podendo ser determinado apenas pelo presente. No entanto, em alguns modelos, como por exemplo, em teoria de controle ou na biologia, o tempo passado exerce uma influência significativa sobre o comportamento futuro. Tais modelos são melhor representados pelas equações diferenciais funcionais do tipo retardado, que levam em consideração um periodo passado.

O estudo de problemas de bifurcação dentro da teoria destas equações constitui o objetivo principal deste trabalho. Mais especificamente, nosso interesse concentra-se no Teorema da Bifurcação de Hopf para equações funcionais autônomas, que mostra uma das formas mais simples em que soluções periódicas não constantes de equações autônomas podem aparecer.

Uma teoria básica sobre as equações funcionais lineares autônomas é apresentada no primeiro capítulo. Essencialmente os teoremas 1.5 e 1.6 fornecem uma visão geométrica do comportamento das soluções. É obtida uma decomposição de $\mathcal{C}$, o espaço das funções contínuas que aplicam o intervalo $[-r, 0]$ no $\mathbf{R}^{\mathbf{n}}$, em soma direta de dois subespaços invariantes, sendo que em um deles 0 fluxo definido pelas soluções pode ser interpretado como o fluxo de uma equação diferencial ordinária num espaço de dimensão finita $e$, sobre o subespaço complementar, as soluções são assintoticamente estáveis. Finalizamos esse capítulo com considerações concernentes a uma equação linear não homogênea e incluimos uma versão da Alternativa de Fredholm.

A parte central deste trabalho encontra-se nos capítulos 2 e 3, onde são apresentadas uma demonstração detalhada do Teorema da Bifurcação de Hopf para equações com retardamento e uma aplicação. A bifurcação de Hopf refere-se ao 
aparecimento de órbitas periódicas a partir de uma solução constante, quando um parâmetro atinge um valor crítico. Mais explicitamente, dada uma familia de equações diferenciais retardadas autônomas da forma

$$
\dot{x}(t)=F\left(\alpha, x_{t}\right),
$$

dependendo de um parâmetro real $\alpha$, e tal que $F(\alpha, 0)=0$ para todo $\alpha$, assumimos que para um certo valor $\alpha_{0}$ do parâmetro $\alpha$ a seguinte situação se verifique: para $\alpha<\alpha_{0}$ as soluções de (1) tendem para zero, quando $t \rightarrow \infty$, e, para $\alpha>\alpha_{0}$, a origem é instável. Assim, o comportamento do fluxo definido pelas soluções de (1) muda abruptamente em $\alpha=\alpha_{0}$. Sob hipóteses convenientes, verifica-se que neste valor do parâmetro surge um ramo de soluções periódicas não constantes da equação (1), sendo ainda possível estimar seu periodo. Isso é provado no Teorema da Bifurcação de Hopf.

No capítulo 3 é feita uma aplicação desse teorema à equação

$$
\dot{x}(t)=\alpha f(x(t), x(t-1))
$$

onde $x=\operatorname{col}\left(x_{1}, x_{2}\right), f=\operatorname{col}\left(f_{1}, f_{2}\right)$ é uma função de classe $\mathcal{C}^{3}$ de $\mathbf{R}^{4}$ em $\mathbf{R}^{2}$ e $\alpha$ é um parâmetro real. A partir do estudo de sua equação característica verificamos, para uma classe particular de funções $f$, a existência de uma infinidade de valores do parâmetro $\alpha$ onde ocorrem bifurcações de Hopf.

Nos Apêndices vistos no final deste trabalho, são apresentados o Teorema da Fórmula da Variação das Constantes e uma descrição do Método de LiapunovSchmidt, que foram instrumentos utilizados no decorrer deste estudo.

Todo conteúdo desenvolvido aqui foi fortemente baseado em [5] e [13]. 


\section{Capítulo 1}

\section{Equações Diferenciais Funcionais Retardadas}

Apresentaremos neste capítulo alguns pré-requisitos que nos serão úteis no desenvolvimento deste estudo.

\section{$1.1 \quad$ Teoria Básica}

Denotaremos por $\mathcal{C}\left([a, b], \mathbf{R}^{\mathbf{n}}\right)$ o espaço de Banach das funções contínuas do intervalo $[a, b]$ no $\mathbf{R}^{\mathbf{n}}$ com a norma

$$
|\phi|=\sup _{\theta \in[a, b]}|\phi(\theta)|
$$

Dado $r>0$ um número real fixo, seja $\mathcal{C}=\mathcal{C}\left([-r, 0], \mathbf{R}^{\mathbf{n}}\right)$.

Se $V$ é um subconjunto de $\mathbf{R} \times \mathcal{C}$, então $\mathcal{C}\left(V, \mathbf{R}^{\mathbf{n}}\right)$ é a classe de todas as funções $f: V \rightarrow \mathbf{R}^{\mathbf{n}}$ que são contínuas e $\mathcal{C}^{0}\left(V, \mathbf{R}^{\mathbf{n}}\right) \subseteq \mathcal{C}\left(V, \mathbf{R}^{\mathbf{n}}\right)$ é o subconjunto das funções contínuas e limitadas de $V$ no $\mathbf{R}^{\mathbf{n}}$, que se torna um espaço de Banach com a norma

$$
|f|_{V}=\sup _{(t, \phi) \in V}|f(t, \phi)| \text {. }
$$

Se $\sigma \in \mathbf{R}, A \geq 0$ e $x \in \mathcal{C}\left([\sigma-r, \sigma+A], \mathbf{R}^{\mathbf{n}}\right)$, para todo $t \in[\sigma, \sigma+A]$ definimos $x_{t} \in \mathcal{C}$ por

$$
x_{i}(\theta)=x(t+\theta),-r \leq \theta \leq 0 \text {. }
$$


Definição 1.1 Seja $D \subset \mathbf{R} \times \mathcal{C}$ e $f: D \rightarrow \mathbf{R}^{\mathrm{n}}$ uma dada função. A relação

$$
\dot{x}(t)=f\left(t, x_{t}\right),
$$

onde "." representa a derivada à direita com relação a t,é uma equação diferencial funcional retardada sobre $D$, que será denotada por $E D F R(f)$, ou simplesmente por EDFR.

As equações diferenciais ordinárias $\dot{x}(t)=F(t, x(t))$, as equações diferenciais diferença $\dot{x}(t)=f\left(t, x\left(t-\tau_{1}(t)\right), \ldots, x\left(t-\tau_{p}(t)\right)\right)$ com $0 \leq \tau_{j}(t) \leq r, j=1, \ldots . p, \mathrm{e}$ as equações integro-diferenciais $\dot{x}(t)=\int_{-r}^{0} g(t, \theta, x(t+\theta)) d \theta$ são casos particulares da equação (1.1).

Definição 1.2 Uma função $x$ é solução da equação (1.1) em $[\sigma-r, \sigma+A)$ se existem $\sigma \in \mathbf{R}$ e $A>0$ tal que $x \in \mathcal{C}\left([\sigma-r, \sigma+A), \mathbf{R}^{\mathbf{n}}\right),\left(t, x_{t}\right) \in D$ e $x(t)$ satisfaz a equação (1.1) para $t \in[\sigma, \sigma+A)$.

Definição 1.3 Dado $(\sigma, \phi) \in D, x(\sigma, \phi, f)$ é uma solução da equação (1.1) com valor inicial $\phi$ em $\sigma$, ou simplesmente, uma solução por $(\sigma, \phi)$, se existir $A>0$ tal que $x(\sigma, \phi, f)$ é solução da equação (1.1) no intervalo $[\sigma-r, \sigma+A) e$ $x_{\sigma}(\sigma, \phi, f)=\phi$.

Se $f: D \rightarrow \mathbf{R}^{\mathbf{n}}$ é contínua, então encontrar uma solução da equação (1.1) por $(\sigma, \phi) \in D$ é equivalente a resolver a equação integral

$$
\begin{aligned}
x(t) & =\phi(0)+\int_{o}^{t} f\left(s, x_{s}\right) d s \\
x_{\sigma} & =\phi
\end{aligned}
$$

Enunciaremos alguns resultados básicos referentes a existência, unicidade $\mathbf{e}$ dependência contínua com relação às condições iniciais, das soluções da equação (1.1). As demonstrações de tais resultados podem ser encontradas em [5, cap.2] .

Teorema 1.1 (Existência): Sejam $\mathbf{\Omega}$ subconjunto aberto em $\mathbf{R} \times \mathcal{C}$ e $f^{0}$ uma dada função em $\mathcal{C}\left(\boldsymbol{\Omega}, \mathbf{R}^{\mathbf{n}}\right)$. Se $(\sigma, \phi) \in \boldsymbol{\Omega}$, então existe uma solução da $E D F R\left(f^{0}\right)$ passando por $(\sigma, \phi)$. Mais geralmente, se $\mathbf{W} \subseteq \mathbf{\Omega}$ é compacto e $f^{0} \in \mathcal{C}\left(\boldsymbol{\Omega}, \mathbf{R}^{\mathbf{n}}\right)$ é dada, então existe uma vizinhança $\mathbf{V} \subseteq \mathbf{\Omega}$ de $\mathbf{W}$ tal que $f^{0} \in \mathcal{C}^{0}\left(\mathbf{V}, \mathbf{R}^{\mathbf{n}}\right)$, existe uma vizinhança $\mathbf{U} \subseteq \mathcal{C}^{0}\left(\mathbf{V}, \mathbf{R}^{\mathbf{n}}\right)$ de $f^{0}$ e $\alpha>0$ tal que, para qualquer $(\sigma, \phi) \in \mathrm{W}, f \in \mathrm{U}$, existe uma solução $x(\sigma, \phi, f)$ da $E D F R(f)$ por $(\sigma, \phi)$, em $[\sigma-r, \sigma+\alpha]$. 
Teorema 1.2 (Dependência contínua): Sejam $\Omega \subseteq \mathbf{R} \times \mathcal{C}$ subconjunto aberto, $\left(\sigma^{0}, \phi^{0}\right) \in \boldsymbol{\Omega}, f^{0} \in \mathcal{C}\left(\boldsymbol{\Omega}, \mathbf{R}^{\mathbf{n}}\right)$ e $x^{0}$ solução da $E D F R\left(f^{0}\right)$ que existe e é única em $\left[\sigma^{0}-r, b\right]$. Consideremos ainda $\mathbf{W}^{0} \subseteq \Omega$ um conjunto compacto definido por $\mathbf{W}^{0}=\left\{\left(t, x_{t}^{0}\right): t \in\left[\sigma^{0}, b\right]\right\}$ e $\mathbf{V}^{0}$ uma vizinhança de $\mathbf{W}^{0}$ na qual $f^{0} e^{\prime}$ limitada. Se $\left(\sigma^{k}, \phi^{k}, f^{k}\right), k=1,2, \ldots$, satisfaz $\sigma^{k} \rightarrow \sigma^{0}, \phi^{k} \rightarrow \phi^{0} e\left|f^{k}-f^{0}\right|_{v^{0}} \rightarrow 0$ quando $k \rightarrow \infty$, entâa existe um $k^{0}$ tal que, para $k \geq k^{0}$, cada solução $x^{k}=x^{k}\left(\sigma^{k}, \phi^{k}, f^{k}\right)$ da EDFR $\left(f^{k}\right)$ por $\left(\sigma^{k}, \phi^{k}\right)$ existe em $\left[\sigma^{k}-r, b\right]$ e $x^{k} \rightarrow x^{0}$ uniformemente em $\left[\sigma^{0}-r, b\right]$. Como pode acontecer de não estarem todos os $x^{k}$ definidos em $\left[\sigma^{0}-r, b\right]$, por $x^{k} \rightarrow x^{0}$ uniformemente em $\left[\sigma^{0}-r, b\right]$ queremos dizer que, para todo $\varepsilon>0$, existe um $k_{1}(\varepsilon)$ tal que $x^{k}(t), k \geq k_{1}(\varepsilon)$ está definido sobre $\left[\sigma^{0}-r+\varepsilon, b\right]$ e $x^{k} \rightarrow x^{0}$ uniformemente em $\left[\sigma^{0}-r+\varepsilon, b\right]$.

Definição 1.4 Sejam $\boldsymbol{\Omega} \subseteq \mathbf{R} \times \mathcal{C}$ e $f \in \mathcal{C}\left(\boldsymbol{\Omega}, \mathbf{R}^{\mathbf{n}}\right)$. Dizemos que $f(i, \phi)$ é localmente Lipschitziana com relação a $\phi$ em $\Omega$ se, para todo subconjunto compacto $\mathbf{W}$ de $\Omega$, existe uma constante real $k=k(\mathbf{W})$ tal que, para cada $(t, \phi),(t, \psi) \in \mathbf{W}$, temos

$$
|f(t, \phi)-f(t, \psi)| \leq k|\phi-\psi|
$$

Teorema 1.3 (Unicidade): Sejam $\mathbf{\Omega}$ subconjunto aberto de $\mathbf{R} \times \mathcal{C},(\sigma, \phi) \in \mathbf{\Omega}$, $f: \Omega \rightarrow \mathbf{R}^{\mathbf{n}}$ contínua e $f(t, \phi)$ localmente Lipschitziana com relação a $\phi$ em $\boldsymbol{\Omega}$. Então existe uma única solução da equação (1.1) por $(\sigma, \phi)$.

Definição 1.5 Suponhamos que na equação (1.1) tenhamos $f$ contínua. Se $x$ é uma solução dessa equação sobre um intervalo $[\sigma, a], a>0, \hat{x}$ é uma continuação de $x$ se existe $u m b>a$ tal que $\hat{x}$ coincide com $x$ em $[\sigma-r, a]$ e satisfaz a equação (1.1) $\mathrm{em}[\sigma, b)$.

Uma solução $x$ é não continuável se nâo existe continuação de $x$. Neste caso, $[\sigma, a]$ é $o$ intervalo maximal de existência da solução $x$.

Sob condições adequadas é possível garantir que a trajetória $\left(t, x_{t}\right)$ em $\mathbf{R} \times \mathcal{C}$ de uma solução da equação (1.1) não continuável sobre $[\sigma, b)$, se aproxima da fronteira do domínio de $f$ quando $t \rightarrow b^{-}$. Essas condições serão estabelecidas no próximo teorema; porém, antes precisaremos de uma definição.

Definição 1.6 Uma aplicação $f$ de um espaço de Banach $X$ em um espaço de Banach $Y$ é compacta se para todo conjunto limitado $A$ em $X$, o fecho do conjunto $f(A):=\{f(x): x \in A\}$ é compacto. Se além disso $f$ for contínua, então $f$ é dita completamente contínua. 
Teorema 1.4 Sejam $\boldsymbol{\Omega}$ aberto em $\mathbf{R} \times \mathcal{C}, f: \boldsymbol{\Omega} \rightarrow \mathbf{R}^{\mathbf{n}}$ completamente continua e $x$ uma solução não continuável da equação (1.1) em $[\sigma-r, b)$. Então, para aualquer conjunto fechado e limitado $\mathbf{U} \mathrm{em} \mathbf{R} \times \mathcal{C}, \mathbf{U} \subset \boldsymbol{\Omega}$, existe um $t_{U}$ tal que $\left(t, x_{t}\right) \notin \mathbf{U}$ para $t_{U} \leq t<b$.

A demonstração deste resultado poderá ser encontrada em [5, pág. 43].

Definição 1.7 Sejam $\mathbf{\Omega} \subseteq \mathbf{R} \times \mathcal{C}$ aberto e $f \in \mathcal{C}\left(\boldsymbol{\Omega}, \mathbf{R}^{\mathbf{n}}\right)$. Para todo $(\sigma, \phi) \in \mathbf{\Omega}$ existem $t_{\sigma, \phi}$ e uma solução não continuável $x$ da $E D F R(f)$ em $\left[\sigma-r, t_{\sigma, \phi}\right)$ por $(\sigma, \phi) ;$ seja $\boldsymbol{\Omega}_{\sigma} \subseteq \mathcal{C}$ definido por $\boldsymbol{\Omega}_{\sigma}=\{\phi \in \mathcal{C}:(\sigma, \phi) \in \boldsymbol{\Omega}\}$. Para cada $\phi \in \boldsymbol{\Omega}_{\sigma}$ e $t \in\left[\sigma, t_{\sigma, \phi}\right)$ definimos a aplicação $T(t, \sigma): \boldsymbol{\Omega}_{\sigma} \rightarrow \mathcal{C}$ por

$$
T(t, \sigma) \phi=x_{t}(\sigma, \phi),
$$

a qual denominaremos aplicação solução da $\operatorname{EDFR}(f)$.

As equações diferenciais funcionais retardadas, embora tenham sua teoria fortemente motivada na das equações diferenciais ordinárias, apresentam algumas características surpreendentemente distintas. Em geral, isto é consequência do espaço de fase ser de dimensão infinita. Este estudo foge do escopo deste trabalho, portanto, em vez de apresentá-lo referímo-nos a [5, cap 3].

\subsection{Equações Lineares Autônomas com Retardamento}

Introduziremos nesta seção as equações diferenciais funcionais lineares autônomas, que serão o objeto de nosso estudo a partir deste momento. Analisaremos inicialmente propriedades da aplicação solução para essa classe particular de equações. Em seguida, com auxílio de resultados de Análise Funcional, obteremos uma decomposição de $\mathcal{C}$ em soma direta de dois subespaços invariantes, sendo que em um deles o fluxo definido pelas soluções pode ser interpretado como o fluxo induzido por uma $E D O$ num espaço de dimensão finita. Finalizando esta seção, é dada uma estimativa das soluções sobre o subespaço complementar.

Definição 1.8 A equação (1.1) é linear se $f(t, \phi)=L(t, \phi)+h(t)$, onde $L(t, \phi)$ $e ́$ linear em $\phi$; é dita linear homogênea se $h \equiv 0$ e linear não homogênea se $h \neq 0$. É autônoma se $f(t, \phi)=g(\phi)$, onde $g$ independe de $t$. 
Consideremos a equação linear autônoma

$$
\dot{x}=L\left(x_{t}\right)
$$

onde $L: \mathcal{C} \rightarrow \mathbf{R}^{\mathbf{n}}$ é linear e contínua.

Pelo Teorema da Representação de Riesz [14, pág. 196], existe uma matriz $\eta(\theta), n \times n,-r \leq \theta \leq 0$, cujos elementos são de variação limitada, tal que

$$
L(\phi)=\int_{-r}^{0}[d \eta(\theta)] \phi(\theta), \quad \phi \in \mathcal{C} .
$$

Se $\phi$ é uma função qualquer em $\mathcal{C}$ e $x(\phi)$ é a única solução da equação (1.3) por $(0, \phi)$, denotamos abreviadamente o opeiaulor solução $T(t): \mathcal{C} \rightarrow \mathcal{C}$, isto é,

$$
T(t) \phi=x_{t}(\phi)
$$

para $t \geq 0$.

Partindo da análise de propriedades da aplicação solução $T(t)$, desejamos entender o comportamento das soluções da equação (1.3) quando elas são interpretadas em $\mathcal{C}$.

Começaremos com o seguinte lema:

Lema $1.1 O$ operador solução $T(t), t \geq 0$, definido pela equação (1.4) satisfaz as seguintes relações:

(i) A familia $\{T(t): t \geq 0\}$ é um semigrupo de operadores lineares, isto $e$, $T(0)=I$ e $T(t+\tau)=T(t) T(\tau)$, para $t, \tau \geq 0$.

(ii) $T(t)$ é limitado para cada $t \geq 0$ e é fortemente contínuo em $[0, \infty)$, isto $e ́, \lim _{\tau \rightarrow t}|T(t) \phi-T(\tau) \phi|=0$, para $t \geq 0$ e $\phi \in \mathcal{C}$.

(iii) $T(t)$ é completamente contínuo para $t \geq r$, isto é, $T(t)$ é contínuo e leva conjunto limitado em conjunto relativamente compacto.

\section{Demonstração:}

(i) Da linearidade da equação (1.3) e do teorema da unicidade, segue que $T(t): \mathcal{C} \rightarrow \mathcal{C}$, definida por (1.4), é uma transformação linear.

Provemos as propriedades de semigrupo: 
Da definição de $T(t)$ vem que

$$
T(0) \phi=x_{0}(\phi)=\phi,
$$

ou seja,

$$
T(0)=I
$$

Mostrar que $T(t+\tau)=T(t) T(\tau)$ é equivalente a mostrar que $x_{t+\tau}=x_{t}\left(x_{\tau}\right)$.

Fixado $\tau$, para todo $\theta \in[-r, 0]$,

$$
x_{t+\tau}(\phi)(\theta)=x(\phi)(t+\tau+\theta)
$$

e

$$
x_{t}\left(x_{\tau}(\phi)\right)(\theta)=x\left(x_{\tau}(\phi)\right)(t+\theta)
$$

Como os primeiros membros coincidem para $t=0$ e $\theta \in[-r, 0]$, segue que os segundos membros $x(\phi)(.+\tau)$ e $x\left(x_{\tau}(\phi)\right)($.$) , são soluções satisfazendo a mesma$ condição inicial em $t=0$.

Pelo teorema da unicidade vem, portanto,

$$
x_{t+\tau}(\phi)=x_{t}\left(x_{\tau}(\phi)\right)
$$

para todo $t, \tau \geq 0$, ou seja,

$$
T(t+\tau)=T(t) T(\tau)
$$

para todo $t, \tau \geq 0$.

(ii)

- $T(t)$ é limitado para cada $t \geq 0$ :

Como $L$ é uma transíormação linear contínua em $\mathcal{C}$, existe uma constante $\ell \geq 0$ tal que

$$
|L(\phi)| \leq \ell|\phi|
$$

para todo $\phi \in \mathcal{C}$.

Da definição de $T(t)$, para cada $t \geq 0$ fixado e $\theta \in[-r, 0]$, temos:

$$
\begin{aligned}
T(t) \phi(\theta)=\phi(t+\theta), & t+\theta \leq 0 \\
T(t) \phi(\theta)=\phi(0)+\int_{0}^{t+\theta} L(T(s) \phi) d s, & t+\theta>0 .
\end{aligned}
$$


Logo,

$$
\begin{aligned}
|T(t) \phi(\theta)|=|\phi(t+\theta)| \leq|\phi|, & \text { se } t+\theta \leq 0 \\
|T(t) \phi(\theta)| \leq|\phi|+\int_{0}^{t+\theta} \ell|T(s) \phi| d s, & \text { se } t+\theta>0
\end{aligned}
$$

de modo que sup $-r \leq \theta \leq 0|T(t) \phi(\theta)| \leq|\phi|+\int_{0}^{t} \ell|T(s) \phi| d s$.

Portanto $|T(t) \phi| \leq|\phi|+\int_{0}^{t} \ell|T(s) \phi| d s$, e a desigualdade de Gronwall implica que

$$
|T(t) \phi| \leq|\phi| e^{\ell t}
$$

para todo $t \geq 0, \phi \in \mathcal{C}$.

Portanto $T(t)$ é limitado para $t \geq 0$.

- $T(t)$ é fortemente contínuo em $[0, \infty)$ :

De (1.5) segue claramente que $\lim _{t \rightarrow 0^{+}}|T(t) \phi-\phi|=0$.

Provemos que $\lim _{\tau \rightarrow t}|T(t) \phi-T(\tau) \phi|=0$ para todo $t \geq 0, \phi \in \mathcal{C}:$

Seja $t \geq 0$ e tomemos $\tau=t+h$. Assim, quando $\tau \rightarrow t^{+}$devemos ter $h \rightarrow 0^{+}$. Então

$$
|T(t) \phi-T(t+h) \phi|=|T(t) \phi-T(t) T(h) \phi| \leq|T(t)||(I-T(h)) \phi| \rightarrow 0
$$

quando $h \rightarrow 0^{+}$, pois t é fixo e $\mathrm{T}(\mathrm{t})$ é limitado.

Se $\tau=t-h \operatorname{com} 0<h<t$, quando $\tau \rightarrow t^{-}$, ainda temos $h \rightarrow 0^{+}$. Assim,

$$
\begin{aligned}
|T(t) \phi-T(t-h) \phi| & =|T(t-h) T(h) \phi-T(t-h) \phi| \\
& \leq|T(t-h)||(T(h)-I) \phi| \rightarrow 0 .
\end{aligned}
$$

quando $h \rightarrow 0^{+}$.

Logo, $|T(t) \phi-T(\tau) \phi| \rightarrow 0$ quando $\tau \rightarrow t$, isto é, $T(\mathrm{t})$ é fortemente contínuo em $[0, \infty)$.

(iii) Como para cada $t$ fixo, $T(t)$ é linear e $|T(t) \phi| \leq e^{\ell t}|\phi|$, temos que $T(t)$ é contínuo.

Resta mostrar que $T(t)$ é compacto para $t \geq r$. 
Seja $S=\{\phi \in \mathcal{C}:|\phi| \leq R\}$. Dado $\psi \in T(t) S, t \geq r$, temos que

$$
|\psi| \leq e^{\ell t} R \text {. }
$$

Portanto, $\psi$ é uniformemente limitada por $e^{\ell t} R$, para $t \geq r$.

Além disso, dado $\varepsilon>0$, tomando $\delta=\varepsilon /\left(\ell e^{\ell i} R\right)$, se $|\theta-\hat{\theta}|<\delta$, então pelo teorema do valor médio, temos:

$|\psi(\theta)-\psi(\hat{\theta})|=|T(t) \phi(\theta)-T(t) \phi(\hat{\theta})|=|x(\phi)(t+\theta)-x(\phi)(t+\hat{\theta})| \leq|\dot{x}(\phi)(\hat{t})||\theta-\hat{\theta}|$

onde $\hat{t} \in(t+\theta, t+\hat{\theta})$ e $\phi \in S$.

Como $|\dot{x}(\phi)(\hat{i})|=\left|L\left(x_{\hat{i}}(\phi)\right)\right| \leq \ell\left|x_{\hat{i}}(\phi)\right| \leq \ell|T(\hat{i})||\phi| \leq \ell e^{\ell \hat{i}} R \leq \ell e^{\ell t} R$, temos

$$
|\psi(\theta)-\psi(\hat{\theta})| \leq \ell e^{\ell t} R \delta=\varepsilon
$$

para toda $\psi \in T(t) S$.

Logo, $T(t) S$ é equicontínuo.

Pelo teorema de Ascoli temos que $T(t) S$ é relativamente compacto para $t \geq r$.

Portanto $T(t)$ é completamente contínuo para $t \geq r$.

As propriedeades (i) e (ii) do lema 1.1 diżem que a familia $T(t), t \geq 0$, é um semigrupo de operadores lineares limitados fortemente contínuos, isto é, um $\mathcal{C}_{0}$ - semigrupo.

Definição 1.9 Dado $\{T(t), t \geq 0\}$ um $C_{0}$-semigrupo, o gerador infinitesimal $A$ de $\{T(t), t \geq 0\}$, é definido como

$$
A \phi=\lim _{t \rightarrow 0^{+}} \frac{1}{t}[T(t) \phi-\phi]
$$

para $\phi \in \mathcal{C}$, quando este limite existe.

Denotaremos por $\mathcal{D}(A)$ o domínio de $A$ e por $\mathcal{R}(A)$ a sua imagem.

Uma expressão conveniente para o gerador infinitesimal $A$ do $C_{0}$-semigrupo $\{T(t), t \geq 0\}$, definido pela equação (1.4), bem como propriedades importantes de $A$, são dadas no próximo lema. 
Lema $1.2 O$ gerador infinitesimal $A$ do $C_{0}-$ semigrupo $T(t), t \geq 0$, definido pela equação (1.4) é dado pela expressão:

$$
A \phi(\theta)= \begin{cases}\dot{\phi}(\theta), & -r \leq \theta<0 \\ L(\phi)=\int_{-r}^{0}[d \eta(\theta)] \phi(\theta), & \theta=0\end{cases}
$$

Além disso, $A$ é um operador fechado com dominio denso em $\mathcal{C}$ e, para qualquer $\phi \in \mathcal{D}(A)$, temos que

$$
\frac{d}{d t} T(t) \phi=T(t) A \phi=A T(t) \phi
$$

\section{Demonstração:}

- A expressão (1.6) segue da definição de $A$ e das equações (1.5).

De fato, para $-r \leq \theta<0$, da primeira equação de (1.5) temos

$$
A \phi(\theta)=\lim _{t \rightarrow 0^{+}} \frac{1}{t}[T(t) \phi(\theta)-\phi(\theta)]=\lim _{t \rightarrow 0^{+}} \frac{1}{t}[\phi(t+\theta)-\phi(\theta)]=\frac{d^{+}}{d \theta} \phi(\theta),
$$

onde $\frac{d^{+}}{d \theta} \phi(\theta)$ é a derivada à direita de $\phi$ em $\theta$.

E se $\theta=0$, a segunda equação de (1.5) nos dá:

$$
A \phi(0)=\lim _{t \rightarrow 0^{+}} \frac{1}{t}[T(t) \phi(0)-\phi(0)]=\lim _{t \rightarrow 0^{+}} \frac{1}{t} \int_{0}^{t} L(T(s) \phi) d s=L(T(0) \phi)=L(\phi) .
$$

Como a imagem de $A$ está contida em $\mathcal{C}, \phi$ pertence ao domínio de $A$ se, e somente se, $\phi$ é contínua e tem derivada à direita contínua em $[-r, 0)$, o que implica que $\dot{\phi}(\theta)$ existe e é contínua em $[-r, 0)$. Mas, ainda $A \phi(0)=L(\phi)$ e, da continuidade de $A \phi$, segue finalmente que $\phi$ pertence ao domínio de $A$ se, e somente se, $\phi$ tem derivada contínua em $[-r, 0]$ e $\dot{\phi}(0)=L(\phi)$.

Assim temos (1.6).

- Se $\phi$ pertence ao domínio de $A$ então para cada $t>0$ fixo, temos: 


$$
\frac{d^{+}}{d t} T(t) \phi=\lim _{h \rightarrow 0^{+}} \frac{T(t+h) \phi-T(t) \phi}{h}=T(t)\left[\lim _{h \rightarrow 0^{+}} \frac{T(h) \phi-\phi}{h}\right]=T(t) A \phi .
$$

e ainda

$$
A T(t) \phi=\lim _{h \rightarrow 0^{+}} \frac{1}{h}[T(h) T(t) \phi-T(t) \phi]=\frac{d^{+}}{d t} T(t) \phi .
$$

Portanto $\frac{d^{+}}{d t} T(t) \phi=T(t) A \phi=A T(t) \phi$.

Para provar (1.7) devemos mostrar que a derivada à esquerda de $T(t) \phi$ existe e é igual a $T(t) A \phi$. Isto segue de:

$$
\begin{aligned}
\lim _{h \rightarrow 0}\left[\frac{T(t) \phi-T(t-h) \phi}{h}-T(t) A \phi\right]= & \lim _{h \rightarrow 0} T(t-\grave{h})\left[\frac{T(h) \phi-\phi}{h}-A \phi\right] \\
& +\lim _{h \rightarrow 0}[T(t-h) A \phi-T(t) A \phi] .
\end{aligned}
$$

Como $|T(t-h)|$ é limitada para $0<h<t$ e $\phi$ está no domínio de $A$, a primeira parcela do segundo membro da igualdade acima tende a zero quando $h \rightarrow 0$ e o mesmo ocorre com a segunda parcela, devido à continuidade forte de $T(t)$.

Logo, $\frac{d^{-}}{d t} T(t) \phi=T(t) A \phi$.

Portanto, $\frac{d}{d t} T(t) \phi=T(t) A \phi=A T(t) \phi$.

- Mostremos que $A$ é fechado:

Seja $\left(\phi_{n}\right)$ uma sequência de funções pertencentes ao domínio de $A$ tal que $\phi_{n} \rightarrow \phi_{0}$ e $A \phi_{n} \rightarrow \psi_{0}$ quando $n \rightarrow \infty$.

Mostraremos que $\phi_{0} \in \mathcal{D}(A)$ e $A \phi_{0}=\psi_{0}$.

Da desigualdade de Gronwall temos

$$
\left|T(s) A \phi_{n}-T(s) \psi_{0}\right| \leq e^{\ell s}\left|A \phi_{n}-\psi_{0}\right|
$$

Como $A \phi_{n} \rightarrow \psi_{0},\left|T(s) A \phi_{n}-T(s) \psi_{0}\right| \rightarrow 0$ uniformemente com relação a $s \in[0, t]$. 
Integrando a relação $\frac{d}{d t} T(t) \phi=T(t) A \phi$ de 0 a $t$ segue que

$$
T(t) \phi-\phi=\int_{0}^{t} T(s) A \phi d s .
$$

Então, para cada $n$ temos $T(t) \phi_{n}-\phi_{n}=\int_{0}^{t} T(s) A \phi_{n} d s$ e, fazendo $n \rightarrow \infty$,

$$
T(t) \phi_{0}-\phi_{0}=\int_{0}^{t} T(s) \psi_{0} d s
$$

Logo,

$$
\lim _{t \rightarrow 0^{+}} \frac{1}{t}\left[T(t) \phi_{0}-\phi_{0}\right]=\lim _{t \rightarrow 0^{+}} \frac{1}{t} \int_{0}^{t} T(s) \psi_{0} d s=\psi_{0} .
$$

Portanto $\phi_{0} \in \mathcal{D}(A)$ e $A \phi_{0}=\psi_{0}$.

- $O$ domínio de $A$ é denso em $\mathcal{C}$ :

Sabemos que $\phi \in \mathcal{D}(A)$ se, e somente se, $\phi$ tem derivada contínua em $[-r, 0]$ e $\dot{\phi}(0)=L(\phi)$. Logo, $\mathcal{D}(A)$ contém o conjunto dos polinômios em $[-r, 0]$, que é denso em $\mathcal{C}$ pelo teorema de Weierstrass [7, pág 132].

. Disto segue a densidade de $\mathcal{D}(A)$ em $\mathcal{C}$.

Como $T(t)$ geralmente não é conhecido, o que se espera é poder analisar propriedades de $T(t)$ usando somente propriedades do gerador infinitesimal $A$, que é conhecido pela expressão (1.6).

Algumas definições são essenciais para esse objetivo.

Definição 1.10 Seja $\mathcal{B}$ um espaço de Banach e $A: \mathcal{B} \rightarrow \mathcal{B}$ um operador linear.

(a) $O$ conjunto resolvente de $A$, que denotaremos por $\rho(A)$, é o conjunto dos valores $\lambda$ do plano complexo para os quais o operador $\lambda I-A$ tem inversa limitada com domínio denso em $\mathcal{B}$. $\sigma(A)$.

(b) $O$ complementar de $\rho(A)$ em $\mathbf{C}$ é o espectro de A, e será denotado por

$O$ espectro de um operador $A$ consiste de três partes mutuamente exclusivas:

(b1) Espectro Residual, denotado por $R \sigma(A)$, que consiste dos $\lambda \in \sigma(A)$ para os quais existe $(\lambda I-A)^{-1}$ mas seu domínio não é denso em $B$. 
(b2) Espectro Contínuo, denotado por $C \sigma(A)$, que consiste dos $\lambda \in \sigma(A)$ para os quais $\lambda I-A$ tem inversa não limitada com domínio denso.

(b3) Espectro Pontual, denotado por $P \sigma(A)$, que consiste dos $\lambda \in \sigma(A)$ para os quais $\lambda I-A$ não tem inversa.

(c) Os elementos $\lambda$ pertencentes ao $P \sigma(A)$ são chamados auto valores de $A$ e qualquer $\phi \in \mathcal{B}, \phi \neq 0$, tal que $(\lambda I-A) \phi=0$ é um auto vetor associado a $\lambda$.

(d) $O$ conjunto das funções $\phi \in \mathcal{B}$ para as quais $A \phi=0$ é chamado núcleo de $A$, denotado por $\mathcal{N}(A)$.

(e) Dado $\lambda \in \sigma(A)$, o autoespaço generalizado de $\lambda$ é o menor subespaço de $\mathcal{B}$ que contém todos os elementos de $\mathcal{B}$ qut pertencem a $\mathcal{N}(\lambda I-A)^{k}, k=1,2, \ldots$ Será denotado por $\mathcal{M}_{\lambda}(A)$.

O lema a seguir nos dá informações acerca da natureza do espectro de $A$, que será de interesse na análise das propriedades do operador $T(t)$. Ainda é obtida neste lema uma decomposição de $\mathcal{C}$ em dois subespaços invariantes, sendo que um deles possui dimensão finita.

Lema 1.3 Se $A$ é definido pela equação (1.6), então $\sigma(A)=P \sigma(A)$ e $\lambda \in \sigma(A)$ se, e somente se, $\lambda$ satisfaz a equação caracteristica

$$
\operatorname{det} \Delta(\lambda)=0 \text { onde } \Delta(\lambda)=\lambda I-\int_{-r}^{0} e^{\lambda \theta} d \eta(\theta) .
$$

As raízes da equaçâo (1.8) tem partes reais limitadas superiormente e, para cada $\lambda \in \sigma(A), \mathcal{M}_{\lambda}(A)$ tem dimensão finita. Além disso, existe um inteiro $k$ tal que $\mathcal{M}_{\lambda}(A)=\mathcal{N}(A-\lambda I)^{k}$ e temos $\mathcal{C}=\mathcal{N}(A-\lambda I)^{k} \oplus \mathcal{R}(A-\lambda I)^{k}$.

\section{Demonstração:}

- Vamos provar que $\sigma(A)=P \sigma(A)$. Para isso mostraremos que todo $\lambda \in \mathbf{C}$ que não satisfaz a equação (1.8) pertence ao $\rho(A)$, e que todo $\lambda$ que satisfaz (1.8) está no $P \sigma(A)$.

Pela definição, $\lambda \in \rho(A)$ se existe $(A-\lambda I)^{-1}$ tal que

(i) $\left|(A-\lambda I)^{-1}\right| \leq M$ para algum $M$, de onde temos que $(A-\lambda I)^{-1}$ é um operador 
linear contínuo, $\mathrm{e}$

(ii) $\mathcal{D}\left((A-\lambda I)^{-1}\right)$ é denso em $\mathcal{C}$.

Ou seja, $\lambda \in \rho(A)$ se, e somente se, para toda $\downarrow$ num subconjunto denso em $\mathcal{C}$, existe $\phi \in \mathcal{D}(A)$ dependendo continuamente de $\psi$, tal que

$$
(A-\lambda I) \phi=\psi \text {. }
$$

Como cada $\phi \in \mathcal{D}(A)$ deve ser continuamente diferenciável e, para $-r \leq \theta \leq 0, \dot{\phi}(\theta)=A \phi(\theta)$, uma solução de (1.9) deve satisfazer

$$
\dot{\phi}(\theta)-\lambda \phi(\theta)=\psi(\theta), \text { para }-r \leq \theta \leq 0 .
$$

Pela fórmula da variação das constantes (veja Apêndice A), temos:

$$
\phi(\theta)=e^{\lambda \theta} b+\int_{0}^{\theta} e^{\lambda(\theta-\xi)} \psi(\xi) d \xi,
$$

$-r \leq \theta \leq 0, \operatorname{com} b=\phi(0)$.

Desde que

$$
\begin{aligned}
\lambda b+\psi(0) & =\dot{\phi}(0)=L(\phi)=\int_{-r}^{0} d \eta(\theta) \phi(\theta) \\
& =\int_{-r}^{0} d \eta(\theta)\left[e^{\lambda \theta} b+\int_{0}^{\theta} e^{\lambda(\theta-\xi)} \psi(\xi) d \xi\right]
\end{aligned}
$$

obtemos

$$
\left[\lambda I-\int_{-r}^{0} d \eta(\theta) e^{\lambda \theta}\right] b=-\psi(0)+\int_{-r}^{0} d \eta(\theta)\left[\int_{0}^{\theta} e^{\lambda(\theta-\xi)} \psi(\xi) d \xi\right],
$$

ou seja

$$
\Delta(\lambda) b=-\psi(0)+\int_{-r}^{0} \int_{0}^{\theta} e^{\lambda(\theta-\xi)} d \eta(\theta) \psi(\xi) d \xi
$$

Se $\lambda$ é tal que det $\Delta(\lambda) \neq 0$, então $\Delta(\lambda)$ é invertível e, de (1.10) e (1.11) segue que

$$
\begin{aligned}
\phi(\theta)= & e^{\lambda \theta}[\Delta(\lambda)]^{-1}\left[-\psi(0)+\int_{-r}^{0} \int_{0}^{\theta} e^{\lambda(\theta-\xi)} d \eta(\theta) \psi(\xi) d \xi\right] \\
& +\int_{0}^{\theta} e^{\lambda(\theta-\xi)} \psi(\xi) d \xi .
\end{aligned}
$$


Assim, se $\lambda \in\{\lambda: \operatorname{det} \Delta(\lambda) \neq 0\}$, obtemos uma solução $\phi \in \mathcal{D}(A)$ de (1.9), que depende continuamente de $\psi$.

Portanto, se $\operatorname{det} \Delta(\lambda) \neq 0$, então $\lambda \in \rho(A)$.

Logo, $\sigma(A) \subseteq\{\lambda: \operatorname{det} \Delta(\lambda)=0\}$.

Mas, se $\lambda$ é tal que det $\Delta(\lambda)=0$, as equações (1.10) e (1.11) implicam que (1.9) tem uma solução não nula para $\psi=0$, isto é $\lambda \in P \sigma(A)$. Provamos dessa forma nossa primeira afirmação.

- As raízes da equação (1.8) têm partes reais limitadas superiormente:

Podemos expressar a equação característica como:

$$
\lambda^{n}+c_{1}(\lambda) \lambda^{n-1}+c_{2}(\lambda) \lambda^{n-2}+\ldots .+c_{n}(\lambda)=0,
$$

onde os coeficientes $c_{j}, j=1,2, \ldots n$, dependem de $\lambda$ através de integrações sobre $[-r, 0]$ de termos da forma $e^{\lambda \theta}$.

Se $\lambda \neq 0$, obtemos

$$
1 \leq \frac{\left|c_{1}(\lambda)\right|}{|\lambda|}+\frac{\left|c_{2}(\lambda)\right|}{|\lambda|^{2}}+\ldots .+\frac{\left|c_{n}(\lambda)\right|}{|\lambda|^{n}}
$$

Todos os $c_{j}, j=1, \ldots n$, são limitados, pois são formados por termos do tipo $s(\lambda)=\int_{-r}^{0} d \eta(\theta) e^{\lambda \theta}$ e, como $e^{\lambda \theta}$ é contínua e $\eta(\theta)$ é de variação limitada,

$$
|s(\lambda)|=\left|\int_{-r}^{0} d \eta(\theta) e^{\lambda \theta}\right| \leq \sup _{\theta \in[-r, 0]}\left|e^{\lambda \theta}\right||V(\eta)|=V(\eta)
$$

onde $V(\eta)$ é a variação total da função $\eta$.

Sendo assim, se para todo $\gamma>0$ existisse uma raiz $\lambda_{0}$ de (1.8) tal que $\Re\left(\lambda_{0}\right)>\gamma$, tomando o limite para $\gamma \rightarrow \infty$ em (1.12), tiraríamos a contradição $1 \leq 0$.

Portanto, existe $\gamma>0$ tal que para toda raiz $\lambda$ de $(1.8), \Re(\lambda) \leq \gamma$.

- Mostraremos agora a parte final do teorema:

Como a função característica det $\Delta(\lambda)$ é inteira, os seüs zeros têm ordem finita: 
Se $\lambda_{0}$ é um zero de ordem $k$ de det $\Delta(\lambda)$, então o operador resolvente, dado por $(A-\lambda I)^{-1}$, tem um polo de ordem $k$ em $\lambda_{0}$.

Sendo $A$ um operador fechado, segue de [14, pág 306] que

$$
\mathcal{C}=\mathcal{N}(A-\lambda I)^{k} \oplus \mathcal{R}(A-\lambda I)^{k},
$$

e o ascendente de $A-\lambda I$ é $k$, de modo que $\mathcal{M}_{\lambda}(A)=\mathcal{N}(A-\lambda I)^{k}$.

Usando a definição de $A$ dada em (1.6), vemos que os elementos de $\mathcal{M}_{\lambda}(A)$ envolvem combinações de funçôes do tipo $a_{j} t^{j} e^{\lambda t}$, onde $j=0, \ldots, k-1$ e os $a_{j}$ 's são vetores $\mathbf{n}$-dimensionais constantes, que satisfazem um sistema de equações lineares. Portanto, $\mathcal{M}_{\lambda}(A)$ tem dimensão finita. Para maiores detalhes veja [9, cap. 10].

Usando esse sistema de equações lineares é possível construir uma base explícita para $\mathcal{M}_{\lambda}(A)$, donde conclui-se que $\operatorname{dim} \mathcal{M}_{\lambda}(A)$ é igual a multiplicidade de $\lambda$ como zero de det $\Delta(\lambda)$. A prova desse fato envolve argumentos de natureza algébrica, podendo ser encontrada em [8].

Se definirmos uma função matricial contínua em $[0, r]$ por

$$
\alpha(s)=e^{-\lambda s} I, 0 \leq s \leq r,
$$

da equação (1.11) obtemos:

$$
-\Delta(\lambda) b=\alpha(0) \psi(0)-\int_{-r}^{0} \int_{0}^{\theta} \alpha(\xi-\theta) d \eta(\theta) \psi(\xi) d \xi:=(\alpha, \psi) .
$$

Da forma como foi definida, $(\alpha, \psi)$ é uma forma bilinear, que será útil para determinar um operador $A^{*}$ com papel relevante nessa teoria.

O lema 1.3 nos permite ter uma idéia do comportamento das soluções da equação (1.3). Vimos que se $\lambda \in \sigma(A)$ então $\mathcal{M}_{\lambda}(A)=\mathcal{N}(A-\lambda I)^{k}$ para algum $k$ e $\operatorname{dim} \mathcal{M}_{\lambda}(A)<\infty$. Podemos então tomar uma base $\left\{\phi_{1}^{\lambda}, \ldots, \phi_{d}^{\lambda}\right\}$ para $\mathcal{M}_{\lambda}(A)$. $\mathrm{O}$ subespaço $\mathcal{M}_{\lambda}(A)$ é invariante sob $A$. Logo, se $\Phi_{\lambda}=\left(\phi_{1}^{\lambda}, \ldots, \phi_{d}^{\lambda}\right)$, existe uma matriz constante $B_{\lambda}, d \times d$, tal que

$$
A \Phi_{\lambda}=\Phi_{\lambda} B_{\lambda}
$$

O único auto valor de $B_{\lambda}$ é $\lambda$.

Da relação (1.13) e da definição de $A$ em (1.6) vem que

$$
\Phi_{\lambda}(\theta)=\Phi_{\lambda}(0) e^{B_{\lambda} \theta},-r \leq \theta \leq 0 .
$$


Do lema 1.2 temos

$$
\frac{d}{d t} T(t) \Phi_{\lambda}-T(t) A \Phi_{\lambda}=0
$$

então,

$$
\frac{d}{d t}\left(T(t) \Phi_{\lambda} e^{-B_{\lambda} t}\right)=0
$$

Integrando de zero a $t$ obtemos

$$
T(t) \Phi_{\lambda}=\Phi_{\lambda} e^{B_{\lambda} t}, \text { para todo } t \geq 0
$$

Assim,

$$
\left[T(t) \Phi_{\lambda}\right](\theta)=\Phi_{\lambda}(0) e^{B_{\lambda} \theta} e^{B_{\lambda} t}=\Phi_{\lambda}(0) e^{B_{\lambda}(t+\theta)},-r \leq \theta \leq 0 .
$$

Vemos dessa forma que podemos definir $T(t)$ sobre $\mathcal{M}_{\lambda}(A)$ para todo $t$ em $\mathbf{R}$.

Portanto, sobre o auto espaço generalizado de um auto valor $\lambda \in \sigma(A)$, a equação (1.3) tem mesma estrutura de uma equação diferencial ordinária.

Ainda, da relação (1.7), tiramos que $\mathcal{R}(A-\lambda I)^{k}$ é invariante sob $T(t)$.

Se considerarmos agora um conjunto finito $\left\{\lambda_{1}, \ldots, \lambda_{p}\right\}$ de auto valores $\mathrm{da}$ equação (1.3), repetindo a análise anterior obtemos:

Teorema 1.5 Se $\Lambda=\left\{\lambda_{1}, \ldots, \lambda_{p}\right\}$ é um conjunto de auto valores da equação (1.3), $\Phi_{\Lambda}=\left(\Phi_{\lambda_{1}}, \ldots, \Phi_{\lambda_{p}}\right)$ onde $\Phi_{\lambda_{j}}$ é base para o auto espaço generalizado de $\lambda_{j} e$ $B_{\Lambda}=\operatorname{diag}\left(B_{\lambda_{1}}, \ldots, B_{\lambda_{p}}\right) \operatorname{com} B_{\lambda_{j}}$ definida por $A \Phi_{\lambda_{j}}=\Phi_{\lambda_{j}} B_{\lambda_{j}}, j=1,2, \ldots p$, então o único auto valor de $B_{\lambda}$, é $\lambda_{j}$ e para cada vetor a que tenha mesma dimensão $q u \in \Phi_{\Lambda}$, a solução $T(t) \Phi_{\Lambda}$ a com valor inicial $\Phi_{\Lambda} a$ em $t=0$ pode ser definida em $\mathbf{R}$ pela relação

$$
\begin{aligned}
T(t) \Phi_{\Lambda} a & =\Phi_{\Lambda} e^{B_{\Lambda} t} a \\
\Phi_{\Lambda}(\theta) & =\Phi_{\Lambda}(0) e^{B_{\Lambda} \theta},-r \leq \theta \leq 0 .
\end{aligned}
$$

Além disso, existe um subespaço $Q_{\Lambda}$ de $\mathcal{C}$ tal que $T(t) Q_{\Lambda} \subseteq Q_{\Lambda}$, para todo $t \geq 0$ e $\mathcal{C}=P_{\Lambda} \oplus Q_{\Lambda}$, onde $P_{\Lambda}=\left\{\phi \in \mathcal{C}: \phi=\Phi_{\Lambda}\right.$ a para algum vetor $\left.a\right\}$.

Assim temos a decomposição de $\mathcal{C}$ em dois subespaços invariantes sob $A$ e $T(t)$, de forma que podemos separar o comportamento das soluções de (1.3) sobre o auto espaço, do comportamento apresentado no espaço complementar. 
Definição $1.11 \lambda_{0}$ é uma raiz característica simples ou um auto valor simples de uma $\operatorname{EDFR}(L)$ se for uma raiz simples da equação característica.

Lema 1.4 Seja $L(\alpha): \mathcal{C} \rightarrow \mathbf{R}^{\mathbf{n}}, \alpha \in \mathbf{R}$, uma familia de operadores lineares limitados e contínuos juntamente com suas primeiras derivadas em $\alpha$. Se $\lambda_{0}$ é um auto valor simples da EDFR(L(0)), então existe $\alpha_{0}>0$ e um auto valor simples $\lambda(\alpha)$ da $E D F R(L(\alpha))$, que é contínuo juntamente com sua primeira derivada, para $|\alpha|<\alpha_{0}$ e $\lambda(0)=\lambda_{0}$.

\section{Demonstração:}

Consideremos a equação

$$
\Delta(\alpha, \lambda) b=0 \text { com } \Delta(\alpha, \lambda)=\lambda I-L(\alpha)\left(e^{\lambda(\alpha)} I\right)
$$

Um número complexo $\lambda$ é uma raiz característica da $E D F R(L(\alpha))$ se, e somente se, existe $b \neq 0$ que satisfaz a equação (1.15).

Se $\lambda_{0}$ é um auto valor simples da $\operatorname{EDFR}(L(0))$, então $\operatorname{dim} \mathcal{N}\left(\Delta\left(0, \lambda_{0}\right)\right)=1 \mathrm{e}$ podemos decompor $\mathbf{C}^{\mathbf{n}}$ como

$$
\mathbf{C}^{\mathbf{n}}=\mathcal{N}\left(\Delta\left(0, \lambda_{0}\right)\right) \oplus \mathcal{R}\left(\Delta\left(0, \lambda_{0}\right)\right)
$$

Associada a esta decomposição temos as projeções $P$ e $I-P$. Reescrevamos a equação (1.15), colocando-a numa forma adequada para aplicar o método de Liapunov - Schmidt (veja Apêndice B).

$$
\Delta\left(0, \lambda_{0}\right) b=\left[\Delta\left(0, \lambda_{0}\right)-\Delta(\alpha, \lambda)\right] b .
$$

Temos $\Delta\left(0, \lambda_{0}\right): \mathbf{C}^{\mathbf{n}} \rightarrow \mathbf{C}^{\mathbf{n}}$ linear e as projeçôes $P, I-P: \mathbf{C}^{\mathbf{n}} \rightarrow \mathbf{C}^{\mathbf{n}}$ tais que

$$
P\left(\mathbf{C}^{\mathbf{n}}\right)=\mathcal{N}\left(\Delta\left(0, \lambda_{0}\right)\right) \text { e }(I-P)\left(\mathbf{C}^{\mathbf{n}}\right)=\mathcal{R}\left(\Delta\left(0, \lambda_{0}\right)\right) .
$$

Fixemos um $b_{0} \in \mathcal{N}\left(\Delta\left(0, \lambda_{0}\right)\right)$.

Seja $b=b_{0}+d, d \in(I-P) \mathbf{C}^{\mathbf{n}}$. Pelo método de Liapunov - Schmidt, existe um operador linear $K: \mathcal{R}\left(\Delta\left(0, \lambda_{0}\right)\right) \rightarrow \mathrm{C}^{\mathrm{n}}$ tal que $\Delta\left(0, \lambda_{0}\right) K=I$ em $\mathcal{R}\left(\Delta\left(0, \lambda_{0}\right)\right)$ e a equação (1.16) é equivalente ao sistema

$$
\left\{\begin{array}{l}
d=K(I-P)\left[\Delta\left(0, \lambda_{0}\right)-\Delta(\alpha, \lambda)\right]\left(b_{0}+d\right) \\
P\left[\Delta\left(0, \lambda_{0}\right)-\Delta(\alpha, \lambda)\right]\left(b_{0}+d\right)=0
\end{array}\right.
$$


que, por sua vez, é equivalente a

$$
\begin{gathered}
\Delta\left(0, \lambda_{0}\right) d=(I-P)\left[\Delta\left(0, \lambda_{0}\right)-\Delta(\alpha, \lambda)\right]\left(b_{0}+d\right) \\
P\left[\Delta\left(0, \lambda_{0}\right)-\Delta(\alpha, \lambda)\right]\left(b_{0}+d\right)=0
\end{gathered}
$$

Comó $\Delta\left(0, \lambda_{0}\right):(I-P) \mathbf{C}^{\mathbf{n}} \rightarrow(I-P) \mathbf{C}^{\mathbf{n}}$ é um isomorfismo e é contínua juntamente com sua primeira derivada, segue do teorema da função implícita a existência de $\delta>0$ e uma única solução $d^{*}=d^{*}\left(\alpha, \lambda, b_{0}\right)$ da equação (1.17) para $\left|\lambda-\lambda_{0}\right|<\delta,|\alpha|<\delta$, que é contínuamente diferenciável em $\alpha, \lambda, b_{0}$ e satisfaz $d^{*}\left(0, \lambda_{0}, b_{0}\right)=0$.

A linearidade de (1.17) ainda implica que, para cada $\alpha$ e $\lambda, d^{*}$ depende linearmente de $b_{0}$, isto é,

$$
d^{*}\left(\alpha, \lambda, b_{0}\right)=D^{*}(\alpha, \lambda) b_{0}
$$

onde $D^{*}(\alpha, \lambda)$ é uma matriz $n \times n$.

Assim, se existir uma solução $b$ de $\Delta(\alpha, \lambda) b=0$, de modo que para $\alpha=0$ e $\lambda=\dot{\lambda}_{0}$ tenhamos $b=b_{0}$, então $b$ deve necessariamente ser definido por

$$
b=b_{0}+D^{*}(\alpha, \lambda) b_{0} \text { para }|\alpha|<\delta \text { e }\left|\lambda-\lambda_{0}\right|<\delta
$$

e da equação (1.18) segue que

$$
f(\alpha, \lambda) b_{0}:=P\left[\Delta\left(0, \lambda_{0}\right)-\Delta(\alpha, \lambda)\right]\left(I+D^{*}(\alpha, \lambda)\right) b_{0}=0
$$

Portanto, a existência de um auto valor $\lambda$ próximo a $\lambda_{0}$, para $\alpha$ próximo de zero, é equivalente à existência de uma solução da equação (1.19).

Temos que

$$
\Delta\left(0, \lambda_{0}\right) \frac{\partial D^{*}\left(0, \lambda_{0}\right)}{\partial \lambda} b_{0}=-(I-P) \frac{\partial \Delta\left(0, \lambda_{0}\right)}{\partial \lambda} b_{0}
$$

Com efeito, de (1.17) segue que

$$
\begin{aligned}
\Delta\left(0, \lambda_{0}\right) D^{*}(\alpha, \lambda) b_{0}= & (I-P)\left[\Delta\left(0 . \lambda_{0}\right)-\Delta(\alpha, \lambda)\right]\left(b_{0}+D^{*}(\alpha, \lambda) b_{0}\right) \\
= & (I-P) \Delta\left(0, \lambda_{0}\right) b_{0}+(I-P) \Delta\left(0, \lambda_{0}\right) D^{*}(\alpha, \lambda) b_{0} \\
& -(I-P) \Delta(\alpha, \lambda)\left(b_{0}+D^{*}(\alpha, \lambda) b_{0}\right) .
\end{aligned}
$$

Desde que $\Delta\left(0, \lambda_{0}\right) b_{0}=0$ e $\Delta\left(0, \lambda_{0}\right) D^{*}(\alpha, \lambda) b_{0} \in \mathcal{R}\left(\Delta\left(0, \lambda_{0}\right)\right)$, obtemos $\Delta\left(0, \lambda_{0}\right) D^{*}(\alpha, \lambda) b_{0}=\Delta\left(0, \lambda_{0}\right) D^{*}(\alpha, \lambda) b_{0}-(I-P) \Delta(\alpha, \lambda)\left(b_{0}+D^{*}(\alpha, \lambda) b_{0}\right)$ 
ou seja

$$
(I-P)\left[\Delta(\alpha, \lambda)\left(b_{0}+D^{*}(\alpha, \lambda) b_{0}\right)\right]=0
$$

Observamos que $\Delta(\alpha, \lambda)$ é uma função matricial. Logo, derivando a expressão acima com relação a $\lambda$, no ponto $\left(0, \lambda_{0}\right)$, obtemos

$$
(I-P)\left[\frac{\partial \Delta\left(0, \lambda_{0}\right)}{\partial \lambda}\left(b_{0}+D^{*}\left(0, \lambda_{0}\right) b_{0}\right)+\Delta\left(0, \lambda_{0}\right) \frac{\partial D^{*}\left(0, \lambda_{0}\right)}{\partial \lambda} b_{0}\right]=0
$$

Sabendo que $D^{*}\left(0, \lambda_{0}\right) b_{0}=0$ e $\Delta\left(0, \lambda_{0}\right) \frac{\partial D^{*}\left(0, \lambda_{0}\right)}{\partial \lambda} b_{0} \in \mathcal{R}\left(\Delta\left(0 . \lambda_{0}\right)\right)$, temos:

$$
(I-P) \frac{\partial \Delta\left(0, \lambda_{0}\right)}{\partial \lambda} b_{0}+\Delta\left(0, \lambda_{0}\right) \frac{d D^{*}\left(0, \lambda_{0}\right)}{d \lambda} b_{0}=0 .
$$

Portanto $\Delta\left(0, \lambda_{0}\right) \frac{\partial D^{*}\left(0, \lambda_{0}\right)}{\partial \lambda} b_{0}=-(I-P) \frac{\partial \Delta\left(0, \lambda_{0}\right)}{\partial \lambda} b_{0}$.

Desta relação segue que

$$
\frac{\partial f\left(0, \lambda_{0}\right)}{\partial \lambda} b_{0}=-P\left[\frac{\partial \Delta\left(0, \lambda_{0}\right)}{\partial \lambda}\right] b_{0} .
$$

Tomemos uma base de $C^{n}$ tal que $\Delta\left(0, \lambda_{0}\right)=\operatorname{diag}(0, B)$ onde $B$ é uma matriz $(n-1) \times(n-1)$ não singular. Assim, podemos escolher $b_{0}=(1,0, \ldots, 0)$ e identificar a projeção $P$ com $P=(1,0, \ldots, 0)$.

Então, se denotarmos por $\alpha(\lambda)$ o elemento que está na primeira linha e primeira coluna da matriz $\Delta(0, \lambda)$, temos que

$$
P[\Delta(0, \lambda)] b_{0}=\alpha(\lambda)
$$

Consequentemente,

$$
P\left[\frac{\partial \Delta\left(0, \lambda_{0}\right)}{\partial \lambda}\right] b_{0}=\left(\operatorname{det} B_{\lambda}\right)^{-1} \frac{\partial \operatorname{det} \Delta\left(0, \lambda_{0}\right)}{\partial \lambda}
$$

onde $B_{\lambda}$ é uma matriz invertível.

Logo,

$$
\frac{\partial f\left(0, \lambda_{0}\right)}{\partial \lambda} b_{0}=-(\operatorname{det} B)^{-1} \frac{\partial \operatorname{det} \Delta\left(0, \lambda_{0}\right)}{\partial \lambda} \neq 0
$$


pois $\lambda_{0}$ é auto valor simples da $\operatorname{EDFR}(L(0))$.

Por $(1.19)$ temos $f\left(0, \lambda_{0}\right)=0$.

Portanto, pelo teorema da função implícità, existe $\bar{\delta}>0$ e uma funçã̃ continuamente diferenciável $\lambda(\alpha), \operatorname{com} \lambda(0)=\lambda_{0}$, tal que $f(\alpha, \lambda(\alpha))=0$, para $|\alpha|<\bar{\delta}$.

Tomando $\alpha_{0}=\min \{\delta, \bar{\delta}\}$, temos a existência de um auto valor $\lambda$ próximo a $\lambda_{0}$ para $|\alpha|<\alpha_{0}$.

Conforme vimos no teorema 1.5 , se $\Lambda$ é um conjunto finito de auto valures da equação (1.3), existe uma decomposição de $\mathcal{C}$ na forma

$$
\mathcal{C}=P_{\Lambda} \oplus Q_{\Lambda}
$$

onde $P_{\Lambda}$ e $Q_{\Lambda}$ são invariantes sob $A$ e $T^{\prime}(t)$, respectivamente, e sobre $P_{\Lambda}$ a equação (1.3) se comporta essencialmente como uma $E D O$.

Queremos agora informações sobre o comportamento de $T(t)$ no subespaço complementar $Q_{\Lambda}$.

Introduziremos um novo operador, em termos do qual será dada uma representação explícita da projeção relativa à decomposição acima.

Se $\mathbf{R}^{\mathbf{n}^{*}}=\left\{x=\left(x_{1}, \ldots, x_{n}\right): x_{i} \in \mathbf{R}\right\}$, definimos $\mathcal{C}^{*}=\mathcal{C}\left([0, r], \mathbf{R}^{\mathbf{n}^{*}}\right)$.

Retomaremos aqui a forma bilinear proveniente da equação (1.11), ou seja, para todo $\alpha \in \mathcal{C}^{*}$ e $\phi \in \mathcal{C}$,

$$
(\alpha, \phi):=\alpha(0) \phi(0)-\int_{-r}^{0} \int_{0}^{\theta} d \eta(\theta) \alpha(\xi-\theta) \phi(\xi) d \xi
$$

Queremos determinar um operador $A^{*}$ que tenha domínio denso em $\mathcal{C}^{*}$, $\mathcal{R}\left(A^{*}\right) \subset \mathcal{C}^{*}$ e tal que

$$
(\alpha, A \phi)=\left(A^{*} \alpha, \phi\right)
$$

para todo $\phi \in \mathcal{D}(A)$ e $\alpha \in \mathcal{D}\left(A^{*}\right)$.

Desta forma, se $\alpha$ tem primeira derivada contínua e $\phi \in \mathcal{D}(A)$, para que a cadeia de igualdades abaixo justifique (1.21), isto é, para que valha

$$
\begin{aligned}
(\alpha, A \phi) & =\alpha(0) \int_{-r}^{0} d \eta(\theta) \phi(\theta)-\int_{-r}^{0} d \eta(\theta) \int_{0}^{\theta} \alpha(\xi-\theta) \dot{\phi}(\xi) d \xi \\
& =\alpha(0) \int_{-r}^{0} d \eta(\theta) \phi(\theta)-\int_{-r}^{0}[\alpha(\xi-\theta) d \eta(\theta) \phi(\xi)]_{0}^{\theta}
\end{aligned}
$$




$$
\begin{aligned}
& +\int_{-r}^{0} \int_{0}^{\theta}\left[\frac{d}{d \xi} \alpha(\xi-\theta)\right] d \eta(\theta) \phi(\xi) d \xi \\
= & {\left[\int_{-r}^{0} \alpha(-\theta) d \eta(\theta)\right] \phi(0)-\int_{-r}^{0} \int_{0}^{\theta}\left[-\frac{d}{d \xi} \alpha(\xi-\theta)\right] d \eta(\theta) \phi(\xi) d \xi } \\
= & \left(A^{*} \alpha, \phi\right)
\end{aligned}
$$

o operador $A^{*}: \mathcal{D}\left(A^{*}\right) \rightarrow C^{*}$ deve ser definido por:

$$
A^{*} \alpha(s)= \begin{cases}-\dot{\alpha}(s), & 0<s \leq r \\ \int_{-r}^{0} \alpha(-\theta) d \eta(\theta) & s=0\end{cases}
$$

com $\mathcal{D}\left(A^{*}\right)$ consistindo de todas as funções $\alpha \in \mathcal{C}^{*}$ que tem primeira derivada contínua e tais que

$$
-\frac{d}{d s} \alpha(0)=\int_{-r}^{0} \alpha(-\theta) d \eta(\theta)
$$

Definição 1.12 operador $A^{*}$ definido em (1.22) satisfazendo a equação (1.21), é chamado operador adjunto formal de $A$ relativo à forma bilinear (1.20).

Consideremos um sistema linear geral do tipo

$$
\dot{x}(t)=L\left(t, x_{t}\right):=\int_{-r}^{0} d_{\theta} \eta(t, \theta) x(t+\theta),
$$

onde $\eta(.,$.$) é uma matriz n \times n$ mensurável em $(t, \theta) \in \mathbf{R} \times \mathbf{R}$, contínua à esquerda em $\theta$ sobre $(-r, 0)$, de variação limitada em $\theta$ no intervalo $[-r, 0]$, que sastisfaz $\eta(t, \theta)=0$, se $\theta \geq 0, \eta(t, \theta)=\eta(t-r)$, se $\theta \leq-r$. Neste caso, sua equação adjunta formal é definida por

$$
y(s)+\int_{s}^{\infty} y(\alpha) \eta(\alpha, s-\alpha) d \alpha=\text { constante }
$$

onde $y \in \mathbf{R}^{\mathbf{n}^{*}}$.

Então, para o caso específico da equação (1.3), a equação adjunta formal fica:

$$
\dot{y}(\tau)=-\int_{-\tau}^{0} y(\tau-\theta) d \eta(\theta)
$$

Se $y$ é solução de (1.23) sobre um intervalo $(-\infty, \sigma+r]$, para cada $\tau \in(-\infty, \sigma)$, definimos $y^{\tau} \in \mathcal{C}^{*}$ por

$$
y^{\tau}(\xi)=y(\tau+\xi), \quad 0 \leq \xi \leq r
$$


Se $x$ é solução de (1.3) em $[\omega-r, \infty)$ e $y$ é solução de $(1.23)$ em $(-\infty, \sigma+r]$, $\sigma>\omega$, pode-se mostrar que $\left(y^{i}, x_{t}\right)$ é constante para todo $t \in[\omega, \sigma]$.

Seguindo o mesmo raciocínio da demonstração do lema 1.3 provamos que o operador $A^{*}$ tem somente espectro pontual e, para todo $\lambda \in \sigma\left(A^{*}\right)$, o auto espaço generalizado de $\lambda$ tem dimensão finita.

Ainda da demonstração do lema 1.3, mais precisamente, das equações (1.9), (1.10), (1.11), segue que $(A-\lambda I) \phi=\psi$ tem solução $\phi$ se, e somente se, para todo vetor $a$ não nulo que satisfaz $a \Delta(\lambda)=0$ tem-se $\left(a e^{-\lambda .}, \psi\right)=0$, onde $(\alpha, \psi)$ é definido em (1.20). Poderíamos expressar essa mesma idéia dizendo que o operador adjunto formal $A^{*}$, de $A$, tem auto funções $e^{-\lambda \theta}, \theta \in[-r, 0]$, e a equação $(A-\lambda I) \phi=\psi$ tem solução se, e somente se, $(\alpha, \psi)=0$, para todas soluções $\alpha$ $\operatorname{de}\left(A^{*}-\lambda I\right) \alpha=0$.

Formalizaremos esse fato, de modo geral, no seguinte lema:

Lema $1.5 A$ equação $(A-\lambda I)^{k} \phi=\psi, \psi \in \mathcal{C}$, tem solução $\phi \in \mathcal{C}$, isto é, $\psi \in \mathcal{R}(A-\lambda I)^{k}$, se e somente se, $(\alpha, \psi)=0$ para todo $\alpha \in \mathcal{N}\left(A^{*}-\lambda I\right)^{k}$.

\section{Demonstração:}

$\cdots$

Introduziremos algumas notações que nos serão convenientes. Definimos:

$$
P_{j+1}(\lambda)=\frac{\Delta^{j}(\lambda)}{j !}, \Delta^{j}(\lambda)=\frac{d^{j} \Delta(\lambda)}{d \lambda^{j}}, j=0.1,2 \ldots,
$$

onde $\Delta(\lambda)$ é a matriz definida em (1.8).

Também definimos as matrizes $\left(A_{k}\right)_{k n \times k n}$ como

$$
A_{k}=\left(\begin{array}{llll}
P_{1} & P_{2} & \ldots & P_{k} \\
0 & P_{1} & \ldots & P_{k-1} \\
\vdots & \vdots & & \vdots \\
0 & 0 & \ldots & P_{1}
\end{array}\right)
$$

Se $(A-\lambda I)^{k} \phi=\psi$ tem solução, então para todo $\theta \in[-r, 0]$,

$$
\left(\frac{d}{d \theta}-\lambda\right)^{k} \phi(\theta)=\psi(\theta)
$$

ou

$$
\phi(\theta)=\sum_{j=0}^{k-1} \gamma_{j+1} \frac{\theta^{j}}{j !} e^{\lambda \theta}+\int_{0}^{\theta} e^{\lambda(\theta-\xi)} \frac{(\theta-\xi)^{k-1}}{(k-1) !} \psi(\xi) d \xi .
$$


onde os $\gamma_{j+1}$ são vetores colunas $\mathrm{n}$-dimensionais, que devem ser determinados de modo que $\phi$ pertença ao domínio de $(A-\lambda I)^{k}$. Determinaremos estas condições sobre os $\gamma_{j}$.

Por indução sobre $m$ temos que

$$
\begin{aligned}
\phi^{(m)}(\theta):=\left(\frac{d}{d \theta}-\lambda\right)^{m} \phi(\theta) & =\sum_{j=0}^{k-m-1} \gamma_{m+j+1} \frac{\theta^{j}}{j !} e^{\lambda \theta} \\
& +\int_{0}^{\theta} e^{\lambda(\theta-\xi)} \frac{(\theta-\xi)^{k-m-1}}{(k-m-1) !} \psi(\xi) d \xi .
\end{aligned}
$$

para $0 \leq m \leq k-1$.

Observemos que $\phi \in \mathcal{D}(A-\lambda I)^{k}$ se, e somente se, $\phi^{(m)} \in \mathcal{D}(A-\lambda I)$, $m=0,1, \ldots, k-1$.

Para $m<k-1, \phi^{(m)} \in \mathcal{D}(A)$ se, e somente se,

$$
P_{1} \gamma_{m+1}+P_{2} \gamma_{m+2}+\ldots+P_{k-m} \gamma_{k}=\int_{-r}^{0} \int_{0}^{\theta} e^{\lambda(\theta-\xi)} \frac{(\theta-\xi)^{k-m-1}}{(k-m-1) !} d \eta(\theta) \psi(\xi) d \xi
$$

Sendo $\phi^{(k-1)}(\theta)=\gamma_{k} e^{\lambda \theta}+\int_{0}^{\theta} e^{\lambda(\theta-\xi)} \psi(\xi) d \xi$, então

$$
\dot{\phi}^{(k-1)}(0)=\gamma_{k} \lambda+\psi(0)
$$

de onde segue que $\phi^{(k-1)} \in \mathcal{D}(A)$ se, e somente se,

$$
P_{1} \gamma_{k}=-\psi(0)+\int_{-r}^{0} \int_{0}^{\theta} e^{\lambda(\theta-\xi)} d \eta(\theta) \psi(\xi) d \xi
$$

Consideremos:

$$
\begin{aligned}
\gamma & :=\operatorname{col}\left(\gamma_{1}, \ldots, \gamma_{k}\right) ; \\
\alpha_{j}(s) & :=\frac{(-s)^{k-j}}{(k-j) !} \epsilon^{-\lambda s}, 0 \leq s \leq r, j=1,2, \ldots, k ; \\
\Psi & :=\operatorname{col}\left(\alpha_{1} I_{n}, \ldots, \alpha_{k} I_{n}\right),
\end{aligned}
$$

onde $I_{n}$ é a matriz identidade $n \times n$. Usando os cálculos acima vemos que existe solução para $(A-\lambda I)^{k} \phi=\psi$ se, e somente se, $\gamma$ satisfaz a equação

$$
A_{k} \gamma=-(\Psi, \psi)=-\operatorname{col}\left(\left(\alpha_{1} I_{n}, \psi\right), \ldots,\left(\alpha_{k} I_{n}, \psi\right)\right)
$$

Da teoria de matrizes temos que a equação acima possui solução se, $e$ somente se, $b(\Psi, \psi)=(b \Psi, \psi)=0$, para todo vetor $b$ tal que $b A_{k}=0$. Mas cálculos semelhantes aos anteriores mostram que $\alpha \in \mathcal{N}\left(A^{*}-\lambda I\right)^{k}$ se, e somente se, $\alpha=b \Psi$, para algum $b$ tal que $b A_{k}=0$. 
Lema 1.6 Para $\lambda \in \sigma(A)$, sejam $\Psi_{\lambda}=\operatorname{col}\left(\psi_{1}, \ldots, \psi_{p}\right), \Phi_{\lambda}=\left(\phi_{1}, \ldots, \phi_{p}\right)$ bases para $\mathcal{M}_{\lambda}\left(A^{*}\right)$ e $\mathcal{M}_{\lambda}(A)$ respectivamente, com $\left(\Psi_{\lambda}, \Phi_{\lambda}\right)=\left(\psi_{i}, \phi_{j}\right), i, j=1, . ., p$. Então $\left(\Psi_{\lambda}, \Phi_{\lambda}\right)$ é não singular, podendo ser tomada como a identidade. A decomposição de $\mathcal{C}$ dada pelo lema 1.3 pode ser escrita explicitamente como

$$
\phi=\phi^{P_{\lambda}}+\phi^{Q_{\lambda}}, \phi^{P_{\lambda}} \in P_{\lambda} \in \phi^{Q_{\lambda}} \in Q_{\lambda},
$$

onde

$$
\begin{gathered}
P_{\lambda}:=\mathcal{M}_{\lambda}(A)=\left\{\phi \in \mathcal{C}: \phi=\Phi_{\lambda} b \text { para algum } p-\text { vetor } b\right\} \\
Q_{\lambda}=\left\{\phi \in \mathcal{C}:\left(\Psi_{\lambda}, \phi\right)=0\right\} \\
\phi^{P_{\lambda}}=\Phi_{\lambda} b, \quad b=\left(\Psi_{\lambda}, \phi\right), \quad \phi^{Q_{\lambda}}=\phi-\phi^{P_{\lambda}} .
\end{gathered}
$$

\section{Demonstração:}

Do lema 1.3 segue que $Q_{\lambda}=\mathcal{R}(A-\lambda I)^{k}$ onde $k$ é o menor inteiro para o qual $\mathcal{M}_{\lambda}(A)=\mathcal{N}(A-\lambda I)^{k}$.

Provemos que $\left(\Psi_{\lambda}, \Phi_{\lambda}\right)$ é não singular:

Suponhamos que exista um p-vetor $a$ tal que $\left(\Psi_{\lambda}, \Phi_{\lambda}\right) a=0$, ou seja,

$$
\left(\begin{array}{cccc}
\left(\psi_{1}, \phi_{1}\right) & \left(\psi_{1}, \phi_{2}\right) & \ldots & \left(\psi_{1}, \phi_{p}\right) \\
\left(\psi_{2}, \phi_{1}\right) & \left(\psi_{2}, \phi_{2}\right) & \ldots & \left(\psi_{2}, \phi_{p}\right) \\
\vdots & \vdots & & \vdots \\
\left(\psi_{p}, \phi_{1}\right) & \left(\psi_{p}, \phi_{2}\right) & \ldots & \left(\psi_{p}, \phi_{p}\right)
\end{array}\right)\left(\begin{array}{c}
a_{1} \\
a_{2} \\
\vdots \\
a_{p}
\end{array}\right)=0
$$

Então $\left(\psi_{j}, \sum_{i=1}^{p} \phi_{i} a_{i}\right)=0, j=1,2, \ldots, p$, isto é, $\left(\psi_{j}, \Phi_{\lambda} a\right)=0$.

Assim, $\left(\Psi_{\lambda}, \Phi_{\lambda} a\right)=0$, e do lema 1.5 vem que $\Phi_{\lambda} a \in \mathcal{R}(A-\lambda I)^{k}$.

Por outro lado, temos também $\Phi_{\lambda} a \in \mathcal{M}_{\lambda}(A)=\mathcal{N}(A-\lambda I)^{k}$. Sendo assim, $\Phi_{\lambda} a \in \mathcal{R}(a-\lambda I)^{k} \cap \mathcal{N}(A-\lambda I)^{k}$ e concluimos, pelo lema 1.3, que $\Phi_{\lambda} a=0$. Portanto $a=0$ e $\operatorname{det}\left(\Psi_{\lambda}, \Phi_{\lambda}\right) \neq 0$.

Ainda do lema 1.3, para cada $\phi \in \mathcal{C}$ temos $\phi=\phi^{P_{\lambda}}+\phi^{Q_{\lambda}}$ onde $P_{\lambda}=\mathcal{M}_{\lambda}(A)$ e $Q_{\lambda}=\mathcal{R}(A-\lambda I)^{k}$.

Obviamente,

$$
P_{\lambda}=\mathcal{M}_{\lambda}(A)=\left\{\phi \in \mathcal{C}: \phi=\Phi_{\lambda} b \text { para algum vetor } b\right\},
$$

uma vez que $\Phi_{\lambda}$ é base para $\mathcal{M}_{\lambda}(A)$ e

$$
\begin{aligned}
Q_{\lambda} & =\mathcal{R}(A-\lambda I)^{k}=\left\{\phi \in \mathcal{C}:(\alpha, \phi)=0 \text { para todo } \approx \in \mathcal{N}\left(A^{*}-\lambda I\right)^{k}\right\} \\
& =\left\{\phi \in \mathcal{C}:\left(\Psi_{\lambda}, \phi\right)=0\right\}
\end{aligned}
$$


Podemos então expressar $\phi \in \mathcal{C}$ como $\phi=\Phi_{\lambda} b+\phi^{Q_{\lambda}}$ para algum p-vetor $b$.

Com isso, e sendo $\left(\Psi_{\lambda}, \Phi_{\lambda}\right)$ a matriz identidade, obtemos:

$$
\left(\Psi_{\lambda}, \phi\right)=\left(\Psi_{\lambda}, \Phi_{\lambda} \bar{b}+\phi^{Q_{\lambda}}\right)=\left(\Psi_{\lambda}, \Phi_{\lambda}\right) b=\imath
$$

Portanto, $\phi^{P_{\lambda}}=\Phi_{\lambda} b, \operatorname{com} b=\left(\Psi_{\lambda}, \phi\right)$ e $\phi^{Q_{\lambda}}=\phi-\phi^{P_{\lambda}}$.

Seja $\Lambda=\left\{\lambda_{1}, \ldots, \lambda_{p}\right\}$ um conjunto de auto valores da equação (1.3). Consideremos $P=P_{\Lambda}$ o subespaço gerado por $\mathcal{M}_{\lambda_{j}}(A), \lambda_{j} \in \Lambda$. Esse subespaço será referido como auto espaço generalizado da equação (1.3) associado a $\Lambda$.

Analogamente definimos $P^{*}=P_{\Lambda}^{*}$ como o auto espaço generalizado da equação adjunta (1.23) associado a $\Lambda$.

Se $\Phi$ e $\Psi$ são bases de $P_{\Lambda}$ e $P_{\Lambda}^{*}$ respectivamente, $(\Psi, \Phi)=I$, então

$$
\mathcal{C}=P_{\Lambda} \oplus Q_{\Lambda}
$$

onde

$$
\begin{aligned}
P_{\Lambda} & =\{\phi \in \mathcal{C}: \phi=\Phi b \text { para algum vetor } b\} \\
Q_{\Lambda} & =\{\phi \in \mathcal{C}:(\Psi, \phi)=0\}
\end{aligned}
$$

e para todo $\phi \in \mathcal{C}$ temos

$$
\begin{aligned}
\phi & =\phi^{P_{\Lambda}}+\phi^{Q_{\Lambda}} \\
\phi^{P_{\Lambda}} & =\Phi(\Psi, \phi) .
\end{aligned}
$$

Definição 1.13 Quando a decomposição de $\mathcal{C}$ dada acima é usada, dizemos que $\mathcal{C}$ é decomposto por $\Lambda$.

Se $\lambda_{0}$ é um auto valor simples da equação (1.3), isto é, se $\lambda_{0}$ é uma raiz da equação (1.8) de multiplicidade um, então $\operatorname{dim} \mathcal{M}_{\lambda_{0}}(A)=1$.

Desde que $\lambda \in \sigma(A)$ se, e somente se, $\lambda \in \sigma\left(A^{*}\right)$ e $\sigma\left(A^{*}\right)=P \sigma\left(A^{*}\right)$ conforme já observamos anteriormente, temos que $\lambda_{0}$ pertence a $\operatorname{P} \sigma\left(A^{*}\right)$ e $\operatorname{dim} \mathcal{M}_{\lambda_{0}}\left(A^{*}\right)=1$.

Sejam $\left\{\phi_{0}\right\}$ e $\left\{\psi_{0}\right\}$ bases de $\mathcal{M}_{\lambda_{0}}(A)=\mathcal{N}\left(A-\lambda_{0} I\right)$ e $\mathcal{M}_{\lambda_{0}}\left(A^{*}\right)=\mathcal{N}\left(A^{*}-\lambda_{0} I\right)$ respectivamente.

$$
\begin{aligned}
\text { Se } \phi_{0} \in \mathcal{M}_{\lambda_{0}}(A) & \Rightarrow\left(A-\lambda_{0} I\right) \phi_{0}(\theta)=0 \text { para todo } \theta \in[-r, 0] \\
& \Rightarrow \frac{d}{d \theta} \phi_{0}(\theta)=\lambda_{0} \phi_{0}(\theta) \Rightarrow \phi_{0}(\theta)=b e^{\lambda \theta}
\end{aligned}
$$


para todo $\theta \in[-r, 0]$.

$$
\begin{aligned}
\text { Se } \psi_{0} \in \mathcal{M}_{\lambda_{0}}\left(A^{*}\right) & \Rightarrow\left(A^{*}-\lambda_{0} I\right) \psi_{0}(\xi)=0 \text { para todo } \xi \in[0, r] \\
& \Rightarrow-\frac{d}{d \theta} \psi_{0}(\xi)=\lambda_{0} \psi_{0}(\xi) \Rightarrow \psi_{0}(\xi)=a e^{-\lambda_{0} \xi}
\end{aligned}
$$

para todo $\xi \in[0, r]$.

Portanto, se $\lambda_{0}$ é auto valor simples da equação (1.3), as bases $\left\{\phi_{0}\right\}$ e $\left\{\psi_{0}\right\}$ são dadas por $\phi_{0}(\theta)=b e^{\lambda_{0} \theta}, \theta \in[-r, 0]$ e $\psi_{0}(\xi)=a e^{-\lambda_{0} \xi}, \xi \in[0, r]$.

Com um cálculo simples vemos que

$$
\left(\psi_{0}, \phi_{0}\right)=a \frac{d \Delta\left(\lambda_{0}\right)}{d \lambda} b
$$

$\operatorname{com} \Delta(\lambda)$ definida em (1.8).

Consideremos uma família $\left\{L(\alpha): \mathcal{C} \rightarrow \mathbf{R}^{\mathbf{n}} ; \alpha \in \mathbf{R}\right\}$ de operadores lineares limitados contínuos juntamente com suas primeiras derivadas em $\alpha$.

Sabemos do lema $1: 4$ que se $\lambda_{0}$ é um auto valor simples da $\operatorname{EDFR}(L(0))$, então, existe um auto valor simples $\lambda(\alpha)$ da $\operatorname{EDF} R(L(\alpha))$, numa vizinhança do zero, contínuo juntamente com sua primeira derivada.

Se forem válidas as hipóteses do lema 1.4, temos os seguintes fatos:

Observação 1.1 Para cada auto valor simples da $\operatorname{EDFR}(L(\alpha))$ sejam

$$
\phi_{\alpha}(\theta)=b(\alpha) e^{\lambda(\alpha) \theta},-r \leq \theta \leq 0 \text { e } \psi_{\alpha}(\xi)=a(\alpha) e^{-\lambda(\alpha) \xi}, 0 \leq \xi \leq r
$$

bases para $\mathcal{M}_{\lambda_{\alpha}}(A)$ e $\mathcal{M}_{\lambda_{\alpha}}\left(A^{*}\right)$, respectivamente, com $\left(\psi_{\alpha}, \phi_{\alpha}\right)=1$.

Então, em virtude de (1.28), uma expressão para a derivada de $\lambda(\alpha)$ com relação a $\alpha$ será dada por

$$
\frac{d \lambda(\alpha)}{d \alpha}=a(\alpha) \frac{d L(\alpha)}{d \alpha}\left(e^{\lambda(\alpha)} I\right) b(\alpha)
$$

Observação 1.2 Suponhamos que $\Lambda(\alpha)=\left\{\lambda_{1}(\alpha), \ldots, \lambda_{p}(\alpha)\right\}$ seja um conjunto finito de auto valores simples da $\operatorname{EDF} R(L(\alpha))$ tal que as seguintes condiçōes estejam satisfeitas:

(i) $\lambda_{2 j-1}(\alpha)=\bar{\lambda}_{2 j}(\alpha), j=1,2, \ldots k$ 
(ii) $\lambda_{j}(\alpha)$ é real para $j=2 k+1, \ldots, p$, $\Phi_{\alpha}, \Psi_{\alpha}$ são bases reais para $\mathcal{M}_{\Lambda(\alpha)}(A)$ e $\mathcal{M}_{\Lambda(\alpha)}\left(A^{*}\right)$ respectivamente com $\left(\Psi_{\alpha}, \Phi_{\alpha}\right)=I$, e $B(\alpha)$ é uma matriz que satisfaz $A(\alpha) \Phi_{\alpha}=\Phi_{\alpha} B(\alpha)$. Então

$$
\frac{d B(\alpha)}{d \alpha}=\Psi_{\alpha}(0) \frac{d L(\alpha)}{d \alpha}\left(\Phi_{\alpha}(0) e^{B(\alpha)}\right)
$$

para todo $\alpha \in \mathbf{R}$.

As demonstrações desses resultados estão em [5, pág 179].

Tentaremos obter agora uma estimativa das soluções sobre o subespaço complementar $Q_{\Lambda}$. Veremos que neste subespaço as soluções da equação (1.3) tendem para zero quando $t$ tende para infinito, ou seja, as soluções de (1.3) são assintoticamente estáveis em $Q_{\Lambda}$.

Precisaremos de um resultado de teoria espectral que admitiremos sem prova.

Lema 1.7 Se o semigrupo $T(t)$ for fortemente contínuo em $[0, \infty)$ com gerador infinitesimal $A$, então $P \sigma(T(t))=e^{t P \sigma(A)}$ mais, possivelmente, $\{0\}$; especificamente, se $\mu=\mu(t) \neq 0$ pertence a $P \sigma(T(t))$ para algum $t$ fixado, então existe $\lambda \in \underline{P} \sigma(A)$ tal que $e^{\lambda t}=\mu$.

Esse resultado é um caso especial do teorema 16.7.2, pág 339 de [6].

Definição $1.14 O$ raio espectral $r_{\sigma}$ de um operador $T$ que aplica um espaço de Banach nele mesmo, é o raio do menor disco, centrado na origem do plano complexo, que contém $\sigma(T)$.

Vale a seguinte fórmula para o raio espectral

$$
r_{\sigma}(T(t))=\lim _{n \rightarrow \infty}\left|T^{n}(t)\right|^{\frac{1}{n}} .
$$

Veja, por exemplo, [11, pág. 355].

Combinando os lemas 1.3, 1.7 e propriedades de operadores compactos, verificamos que para cada $\beta \in \mathbf{R}$ existe apenas um número finito de $\lambda$ 's em $\sigma(A)$ que tem parte real maior ou igual a $\beta$. De fato:

Suponhamos, por um momento, que exista $\beta$ para o qual se tenha infinitos $\lambda$ 's em $\sigma(A)$ com $\Re \lambda \geq \beta$. Seja então $\left(\lambda_{n}\right)_{n \in N}$ uma sequência de elementos de $\sigma(A)$ que satisfazem $\Re \lambda_{n} \geq \beta$ e tal que $\lambda_{n} \neq \lambda_{m}$, se $n \neq m, n, m \in \mathbf{N}$. 
Pelo lema 1.3 temos que $\sigma(A)=P \sigma(A)$. Logo, $\lambda_{n} \in P \sigma(A)$ para todo $n \in \mathbf{N}$. Como $T(r)$ é compacto, de acordo com [14, teo 5.5F,pág 281], por exemplo, segue que se $\mu \in \sigma(T(r))$ então $\mu \in P \sigma(T(r))$. Ainda do lema 1.7, $P \sigma(T(r))$ é dado por $\hat{\boldsymbol{c}}^{+P \sigma(A)}$ mais, possivelmente, $\{0\}$.

Tomamos então uma sequência $\left(\mu_{n}\right)$ de pontos de $P \sigma(T(r))$ tal que, para todo $n \in \mathbf{N}, \mu_{n}=e^{r \lambda_{n}}$. Se todos os $\mu_{n}$ forem distintos, temos uma infinidade de $\mu_{n} \in P \sigma(T(r))$ distintos, tais que

$$
\left|\mu_{n}\right|=\left|e^{r \lambda_{n}}\right|=e^{r \Re \lambda_{n}} \geq e^{r \beta} .
$$

Portanto, $\operatorname{P\sigma }(T(r))$ tem ponto de acumulação fora da bola de centro zero e raio $e^{\tau \beta}$, o que é absurdo, pois como $T(r)$ é compacto, $P \sigma(T(r))$ não pode ter ponto de acumulação diferente de zero. Veja [14, Teo 5.5G, pág 281], por exemplo.

Se existir $n \neq m$ tal que $\mu_{n}=\mu_{m}$, então $e^{r \lambda_{n}}=e^{r \lambda_{m}}$, e isso implica que $\lambda_{n}=\lambda_{m}+2 k \pi i / r$. Para $t>r$ obtemos

$$
e^{\lambda_{n} t}=e^{\left(\lambda_{m}+2 k \pi i / r\right) t} \neq e^{\lambda_{m} t} .
$$

Então, tomamos $\mu_{n}=\mu_{n}(t)=e^{\lambda_{n} t} \in P \sigma(T(t)), t>r$, e chegamos na contradição anterior.

Podemos agora demonstrar o seguinte resultado:

Teorema 1.6 Dado um número real $\beta$, seja $\Lambda=\Lambda(\beta)=\{\lambda \in \sigma(A): \Re \lambda \geq \beta\}$ e suponha que $\mathcal{C}$ é decomposto por $\Lambda$ na forma $\mathcal{C}=P_{\Lambda} \oplus Q_{\Lambda}$. Então existem constantes positivas $M$ e $\gamma$ tais que

$$
\begin{aligned}
& \left|T(t) \phi^{P_{\Lambda}}\right| \leq M e^{(\beta+\gamma) t}\left|\phi^{P_{\Lambda}}\right|, \quad t \leq 0 \\
& \left|T(t) \phi^{Q_{\Lambda}}\right| \leq M e^{(\beta-\gamma) t}\left|\phi^{Q_{\Lambda}}\right|, \quad t \geq 0 .
\end{aligned}
$$

\section{Demonstração:}

Como foi observado acima, $\sigma(T(r))-\{0\}=e^{r \sigma(A)}$ e existe apenas um número finito de auto valores de $A$ no semiplano determinado pela reta $x=\beta$. Via aplicação exponencial, $z \mapsto e^{r z}$, essa reta é levada sobre a esfera de raio $e^{\beta r}$.

Logo, se considerarmos $\Re \sigma(A) \neq \beta$, existirá apenas um número finito de auto valores de $T(r)$ fora dessa esfera, enquanto os demais estão dentro da mesma, ou seja, $\sigma(T(r)) \cap\left\{\lambda:|\lambda|=e^{\beta r}\right\}=\emptyset$. 
Para simplificar a notação, consideraremos

$$
P=P_{\Lambda} \text { e } Q=Q_{\Lambda}
$$

Podemos então decompor $\sigma(T(r))$ como

$$
\sigma(T(r))=\sigma_{r}^{Q} \cup \sigma_{r}^{P}
$$

onde $\sigma_{r}^{Q}=\sigma(T(r)) \cap\left\{\lambda:|\lambda|<e^{\beta r}\right\}$ e $\sigma_{r}^{P}=\sigma(T(r)) \cap\left\{\lambda:|\lambda|>e^{\beta r}\right\}$.

Observamos que $\sigma\left(\left.T(r)\right|_{Q}\right)=\sigma_{r}^{Q}$ e $\sigma\left(\left.T(r)\right|_{P}\right)=\sigma_{r}^{P}$.

Tomando $\gamma_{1}=d / 2$ onde $d=\operatorname{dist}\left(\left\{\lambda:|\lambda| \geq e^{\beta r}\right\}, \sigma_{r}{ }^{Q}\right)$, temos

$$
\sigma_{r}^{Q} \subset\left\{\lambda:|\lambda| \leq e^{\left(\beta-\gamma_{1}\right) r}\right\} \text {. }
$$

Então $r_{\sigma}\left(\left.T(r)\right|_{Q}\right)<e^{\left(\beta-\gamma_{1}\right) r}$. Veremos que esta relação se mantém para qualquer $t \geq 0$.

Para cada $k \in \mathbf{N}, k t=r n_{k}+\tau_{k}$ com $0 \leq \tau_{k}<r$, e existe uma constante $\ell$ tal que

$$
|T(t)| \leq e^{\ell t} \leq e^{\ell r}:=L \text { para } 0 \leq t<r .
$$

Então

$$
\begin{aligned}
& r_{\sigma}\left(\left.T(t)\right|_{Q}\right)=\left.\lim _{k \rightarrow \infty}\left|T^{k}(t)\right|_{Q}\right|^{\frac{1}{k}} \\
& =\left.\left.\lim _{k \rightarrow \infty}\left|T\left(r n_{k}\right)\right|_{Q}\right|^{\mid\left(n_{k} r+\tau_{k}\right)^{-1}}\left|T\left(\tau_{k}\right)\right|_{Q}\right|^{\mid\left(n_{k} r+\tau_{k}\right)^{-1}} \\
& \leq\left.\lim _{k \rightarrow \infty}\left|T\left(r n_{k}\right)\right|_{Q}\right|^{n_{k}^{-1} t /\left(r+\tau_{k} n_{k}^{-1}\right)} L^{t / n_{k}\left(r+\tau_{k} n_{k}^{-1}\right)} \\
& =r_{\sigma}\left(\left.T(r)\right|_{Q}\right)^{t r^{-1}}<e^{\left(\beta-\gamma_{1}\right) r_{r}^{\frac{1}{r}}}=e^{\left(\beta-\gamma_{1}\right) t} \text {. }
\end{aligned}
$$

Por outro lado, temos que

$$
\sigma\left(\left.T(r)\right|_{P}\right) \subset\left\{\lambda:|\lambda|>e^{\beta r}\right\}
$$

e portanto, $\left\{\lambda:|\lambda|<e^{\beta r}\right\} \subset \rho\left(\left.T(r)\right|_{P}\right)$.

Como $\left.T(-t)\right|_{P}=\left(\left.T(t)\right|_{P}\right)^{-1}, t \geq 0$,

$$
\left\{\lambda:|\lambda| \geq e^{-\beta r}\right\} \subset \rho\left(\left.T(-r)\right|_{P}\right),
$$

ou seja,

$$
\sigma\left(\left.T(-r)\right|_{P}\right) \subset\left\{\lambda:|\lambda|<e^{-\beta r}\right\}
$$


e portanto $r_{\sigma}\left(\left.T(-r)\right|_{P}\right)<e^{-\beta r}$.

Com cálculos análogos aus anteriores verificamos a existência de $\gamma_{2}>0$ tal que $r_{\sigma}\left(\left.T(-r)\right|_{P}\right)<e^{-\left(\beta+r_{2}\right) r}$ e estendemos esta relação a todo $t \geq 0$, isto é,

$$
r_{\sigma}\left(\left.T(-t)\right|_{P}\right)<e^{-\left(\beta+\gamma_{2}\right) t}, \quad t \geq 0 .
$$

Seja $\gamma:=\min \left\{\gamma_{1}, \gamma_{2}\right\}$

Como $r_{\sigma}\left(\left.T(t)\right|_{Q}\right)=\left.\lim _{k \rightarrow \infty}\left|T^{k}(t)\right|_{Q}\right|^{\frac{1}{k}}<e^{\left(\beta-\gamma_{1}\right) t}, t \geq 0$, então, para algum $k_{0}$,

$$
|T(k t)|_{Q} \mid<e^{k\left(\beta-\gamma_{1}\right) t}
$$

para $k \geq k_{0}$.

Seja $M_{1}$ tal que

$$
|T(k t)|_{Q} \mid \leq M_{1} e^{k\left(\beta-\gamma_{1}\right) t} \text { para } k \geq 0
$$

Então,

$$
|T(t)|_{Q} \mid \leq M_{1} e^{\left(\beta-\gamma_{1}\right) t} \leq M_{1} e^{(\beta-\gamma) t}, t \geq 0 .
$$

Do mesmo modo, tomando a relação $r_{\sigma}\left(\left.T(t)\right|_{P}\right)<e^{\left(\beta+\gamma_{2}\right) t}, t \leq 0$, existe $M_{2}$ para o qual

$$
|T(t)|_{P} \mid \leq M_{2} e^{(\beta+\gamma) t} .
$$

Portanto, tomando $M=\max \left\{M_{1}, M_{2}\right\}$, a prova do teorema fica completa.

Em particular, escolhendo $\beta=0$ no teorema 1.6, podemos separar os auto valores quie tem parte real negativa dos que tem parte real positiva. Considerando as raízes características $\lambda$ da equação (1.8) com $\Re \lambda \leq 0$ obtemos um corolário importante do teorema 1.6 que se refere a estabilidade exponencial assintótica que desejamos:

Corolário 1.1 Se todas as raizes da equação característica (1.8) tem partes reais negativas, então existem constantes positivas $k$ e $\gamma$ tais que

$$
|T(t) \phi| \leq k e^{-\gamma t}|\phi|, \quad t \geq 0
$$

para todo $\phi \in \mathcal{C}$. 


\section{Demonstração:}

Tomando $\beta=0$ no teorema 1.6 , temos que o conjunto $\Lambda$ é vazio e portanto $P_{\Lambda}=\{0\}$.

Logo, $\mathcal{C}=Q_{\Lambda}$ e pelo teorema existem $k>0$ e $\gamma>0$ tais que

$$
|T(t) \phi| \leq k e^{-\gamma t}|\phi|, \quad t \geq 0,
$$

para todo $\phi \in \mathcal{C}$.

\subsection{Equação Linear Autônoma Forçada}

Utilizaremos a decomposição descrita na seção anterior para obter resultados referentes a equações lineares não homogêneas do tipo

$$
\dot{x}(t)=L\left(x_{t}\right)+f(t)
$$

onde $f:[0, \infty) \rightarrow \mathbf{R}^{\mathbf{n}}$ é uma função Lebesgue-integrável em cada conjunto compacto de $[0, \infty)$.

Daremos uma condição necessária e suficiente para que essa equação tenha uma solução $w$-periódica, desde que o termo forçante $f$ seja $w$-periódico. Tal condição encontra-se no teorema da Alternativa de Fredholm, que será demonstrado nesta seção. Enfatizamos ainda que este teorema será de grande importância posteriormente.

Para cada $\sigma \in \mathbf{R}$, pela Fórmula da Variação das Constantes, uma solução $x$ da equação não homogênea (1.30) com valor inicial $x_{\sigma}$ em $\sigma$ deve satisfazer

$$
x_{t}=T(t-\sigma) x_{\sigma}+\int_{\sigma}^{t} T(t-s) X_{0} f(s) d s, t \geq \sigma,
$$

onde

$$
X_{0}(\theta)=\left\{\begin{array}{lc}
0, & -r \leq \theta<0 \\
I, & \theta=0
\end{array}\right.
$$

Consideremos $\Lambda=\Lambda_{0} \cup \Lambda_{1}$, com

$$
\begin{aligned}
& \Lambda_{0}=\{\lambda \in \sigma(A): \Re \lambda=0\} \\
& \Lambda_{1}=\{\lambda \in \sigma(A): \Re \lambda>0\}
\end{aligned}
$$


Seja $\mathcal{C}$ decomposto por $\Lambda$ na forma

$$
\mathcal{C}=P_{0} \oplus P_{1} \oplus Q
$$

onde $P_{0}$ e $P_{1}$ são os auto espaços generalizados da equá̧̌̃ homogênea

$$
\dot{x}(t)=L\left(x_{t}\right)
$$

associados com $\Lambda_{0}$ e $\Lambda_{1}$ respectivamente.

Se $x_{i}=x_{t}^{P_{0}}+x_{t}^{P_{1}}+x_{t}^{Q}$, considerando $\Phi_{j}$ uma base para $P_{j}$ e $\Psi_{j}$ uma base para $P_{j}^{*}, j=0,1$, então a equação (1.31) é equivalente a

$$
\begin{aligned}
& x_{t}^{P_{0}}=T(t-\sigma) x_{\sigma}^{P_{0}}+\int_{\sigma}^{t} T(t-s) X_{0}^{P_{0}} f(s) d s \\
& x_{t}^{P_{1}}=T(t-\sigma) x_{\sigma}^{P_{1}}+\int_{\sigma}^{t} T(t-s) X_{0}^{P_{1}} f(s) d s \\
& x_{t}^{Q}=T(t-\sigma) x_{\sigma}^{Q}+\int_{\sigma}^{t} T(t-s) X_{0}^{Q} f(s) d s,
\end{aligned}
$$

$t \geq \sigma$, onde $X_{0}^{P_{j}}=\Phi_{j} \Psi_{j}(0)$ e $X_{0}^{Q}=X_{0}-X_{0}^{P_{0}}-X_{0}^{P_{1}}$.

Maiores detalhes dessa decomposição são encontrados na parte da teoria sobre a decomposição da equação homogênea (1.32), que pode ser vista em [5, pág 185188].

Definamos ainda

$$
\mathcal{P}_{w}=\left\{f: \mathbf{R} \rightarrow \mathbf{R}^{\mathbf{n}} ; f \text { é contínua e } w \text {-periódica }\right\}
$$

com a norma $|f|_{P_{w}}=\sup _{0 \leq t \leq w}|f(t)|$.

$$
\mathcal{B}\left(\mathbf{R}^{+}\right)=\left\{f: \mathbf{R}^{+} \rightarrow \mathbf{R}^{\mathbf{n}} ; f \text { é contínua e limitada em } \mathbf{R}^{+}\right\}
$$

com norma $|f|_{B\left(R^{+}\right)}=\sup _{t \in R^{+}}|f(t)|$.

$\mathcal{B}\left(\mathbf{R}^{-}\right)$e $\mathcal{B}(\mathbf{R})$ são definidos de modo análogo, juntamente com suas respectivas normas.

Estes espaços são todos de Banach.

Se $\mathcal{B}$ é uma das classes $\mathcal{B}\left(\mathbf{R}^{+}\right), \mathcal{B}\left(\mathbf{R}^{-}\right)$, ou $\mathcal{B}(\mathbf{R})$ e $f \in \mathcal{B}$, uma condição necessária para que a equação (1.30) tenha solução em $B$ é dada no lema a seguir:

Lema 1.8 Se a equação (1.30) tem uma solução em $B$ para toda $f \in \mathcal{B}$, entâo não existem auio valores da equação característica associada à equação (1.32) sobre o eixo imaginário, isto é, $\Lambda_{0}$ é vazio e $P_{0}=\{0\}$. 
A demonstração desse resultado poderá ser encontrada em [5, pág 205].

Lema 1.9 Suponhamos que $\Lambda_{0}$ é vazio. As equaçōes (1.34) e (1.35) possuem soluções únicas, $x_{i}^{P_{1}}, x_{t}^{Q}$, que são limitadas para $t \in \mathbf{R}$. Estas funções são dadas por

$$
\begin{aligned}
x_{t}^{Q} & =\int_{-\infty}^{t} T(t-s) X_{0}^{Q} f(s) d s \\
x_{t}^{P_{1}} & =\int_{\infty}^{t} T(t-s) X_{0}^{P_{1}} f(s) d s, \quad t \in \mathbf{R} .
\end{aligned}
$$

Além disso, $x_{t}^{Q}, x_{t}^{P_{1}}$, são funções lineares contínuas em $\mathcal{B}$, isto é, existe uma constante $M>0$ tal que

$$
\left|x_{t}^{P_{1}}\right|,\left|x_{t}^{Q}\right| \leq M \sup _{\bullet \in \mathbf{R}}|f(s)|, \quad t \in \mathbf{R} .
$$

Também, se f for w-periódica, então

$$
x_{t}^{P_{1}}=x_{t+w}^{P_{1}} \text { e } x_{t}^{Q}=x_{t+w}^{Q}
$$

para todo $t$.

\section{Demonstração:}

Se $x$ é solução de (1.31) sobre $(-\infty, \infty)$, então (1.34) e (1.35) são válidas para todo $\sigma \in(-\infty, \infty)$.

Consideremos primeiramente a função $x_{t}^{Q}$.

Sendo $\Lambda=\{\lambda \in \sigma(A): \Re \lambda>0\}$, pelo teorema 1.6 existem $k>0$ e $\alpha>0$ tais que

$$
\left|T(t) \phi^{Q}\right| \leq k e^{-\alpha t}\left|\phi^{Q}\right|, \quad t \geq 0
$$

para toda $\phi^{Q} \in Q$.

Consequentemente, para $t \geq \sigma$,

$$
\left|T(t-\sigma) x_{\sigma}^{Q}\right| \leq k e^{-\alpha(t-\sigma)}\left|x_{\sigma}^{Q}\right|
$$

para todo $\sigma \in(-\infty, \infty)$.

Se $\left|x_{\sigma}^{Q}\right|$ é uniformemente limitada em $\sigma$, vem de (1.38) que $T(t-\sigma) x_{\sigma}^{Q} \rightarrow 0$ quando $\sigma \rightarrow-\infty$, de modo que $x_{t}^{Q}$ satisfaz (1.36). 
Podemos escolher $k$ e $\alpha$ tais que

$$
\left|T(t) X_{0}^{Q}\right| \leq k e^{-\alpha t}, t \geq 0 .
$$

Então, tomando $M=k / \alpha$ obtemos a estimativa (1.37) para $\left|x_{\imath}^{Q}\right|, t \in \mathbf{R}$.

Isto mostra que de fato existe uma única solução $x_{t}^{Q}$ da equação (1.35), limitada, que é definida em (1.36).

Ainda, como

$$
x_{t}^{Q}=\int_{-\infty}^{0} T(-u) X_{0}^{Q} f(t+u) d u
$$

segue imediatamente que se $f(t+w)=f(t)$, então $x_{t+w}^{Q}=x_{t}^{Q}$ para todo $t$.

Para $x_{t}^{P_{1}}$ o procedimento é ligeiramente diferente.

Sejam $\Phi$ uma base para $P_{1}, \Psi$ uma base para o auto espaço generalizado da equação adjunta associada ao conjunto $\Lambda,(\Psi, \Phi)=I$ e seja $B$ a matriz cujos auto valores coincidem $\operatorname{com} \Lambda_{1}$, determinada por $A \Phi=\Phi B$.

Dado $\phi \in \mathcal{C}, \phi^{P_{1}}=\Phi(\Psi, \phi)$ e $T(t)^{P_{1}}=\Phi e^{B t}(\Psi, \phi)$. Então, de (1.34) obtemos

$$
\begin{aligned}
0 & =\Phi\left[\left(\Psi, x_{t}\right)-e^{B(t-\sigma)}\left(\Psi, x_{\sigma}\right)-\int_{\sigma}^{t} e^{B(t-s)}\left(\Psi, X_{0}\right) f(s) d s\right] \\
& =\Phi\left[\left(\Psi, x_{t}\right)-e^{B(t-\sigma)}\left(\Psi, x_{\sigma}+\int_{\sigma}^{t} e^{B(\sigma-s)} X_{0} f(s) d s\right)\right]
\end{aligned}
$$

Portanto

$$
\left(\Psi, x_{t}\right)=e^{B(t-\sigma)}\left(\Psi, x_{\sigma}+\int_{\sigma}^{t} e^{B(\sigma-s)} X_{0} f(s) d s\right)
$$

Se $x_{t}^{P_{1}}$ é limitado, $\left(\Psi, x_{t}\right)=\left(\Psi, x_{t}^{P_{1}}\right)$ também o é.

Logo, fazendo $t$ tender a $\infty$ na última equação, obtemos

$$
\left(\Psi, x_{\sigma}+\int_{\sigma}^{t} e^{B(\sigma-s)} X_{0} f(s) d s\right) \rightarrow 0
$$

Multiplicando por $\Phi$, isso nos dá

$$
x_{\sigma}^{P_{1}}+\int_{\sigma}^{t} T(\sigma-s) X_{0}^{P_{1}} f(s) d s \rightarrow 0
$$

quando $t \rightarrow \infty$, o que implica, finalmente, que

$$
x_{t}^{P_{1}}=\int_{\infty}^{t} T(t-s) X_{0}^{P_{1}} f(s) d s
$$


para todo $t \in(-\infty, \infty)$.

Verificamos assim a relação (1.36). As demais afirmações do lema são concluidas de modo análogo às anteriores.

Estamos agora em condições de demonstrar a Alternativa de Fredholm.

Consideremos $\tilde{\pi}: \mathcal{P}_{\boldsymbol{w}} \rightarrow \mathcal{P}_{\boldsymbol{w}}$ uma projeção contínua de $\mathcal{P}_{\boldsymbol{w}}$ sobre as soluções $w$-periódicas da equação (1.32).

Teorema 1.7 (Alternativa de Fredholm) $S e f \in \mathcal{P}_{w}$, então o sistema (1.30) tem uma soluçâo em $\mathcal{P}_{w}$ se e somente se

$$
\int_{0}^{v} y(t) f(t) d t=0
$$

para toda solução w-periódica da equação adjunta formal

$$
\dot{y}(s)=-\int_{-\tau}^{0} y(s-\theta) d \eta(\theta)
$$

Além disso, existe uma projeção contínua $J: \mathcal{P}_{w} \rightarrow \mathcal{P}_{w}$ tal que o conjunto das $f \in \mathcal{P}_{w}$ que satisfazem a relação $(1.39)$ é $(I-J) \mathcal{P}_{w}$ e existe um operador linear contínuo $K:(I-J) \mathcal{P}_{w} \rightarrow(I-\tilde{\pi}) \mathcal{P}_{w}$ tal que $K f$ é uma solução da equação (1.30) para cada $f \in(I-J) \mathcal{P}_{\boldsymbol{w}}$.

\section{Demonstração :}

Consideremos a decomposição de $\mathcal{C}$ na forma $\mathcal{C}=P_{0} \oplus P_{1} \oplus Q$, de acordo com a notação anterior.

Se $x$ é solução da equação (1.30) e $x_{t}=x_{t}^{P_{0}}+x_{t}^{P_{1}}+x_{t}^{Q}$, as equações (1.33), (1.34), (1.35) devem ser satisfeitas.

Pelo lema 1.9, as equações (1.34) e (1.35) têm soluções $w$-periódicas únicas para cada $f \in \mathcal{P}_{w}$, que são dadas explicitamente por (1.36). Logo, basta considerarmos a equação (1.33).

Sejam $\Phi_{0}$ uma base para $P_{0}$ e $\Psi_{0}$ uma base para o auto espaço generalizado da equação adjunta associado com $\Lambda_{0}$, tal que $\left(\Psi_{0}, \Phi_{0}\right)=I$, e seja $B$ uma matriz tal que $A \Phi_{0}=\Phi_{0} B$, onde $A$ é o gerador infinitesimal associado à equação (1.32). Observemos que os auto valores de $B$ coincidem com os elementos de $\Lambda_{0}$ e assim têm partes reais iguais a zero. 
Então, se $x_{t}^{P_{0}}=\Phi_{0} z(t), z(t)$ será solução da equação

$$
\dot{z}(t)=B z(t)+\Psi_{0}(0) f(t)
$$

como pode ser visto em [3, pág 121$]$.

$\mathrm{Da}$ teoria de sistemas lineares de equações diferenciais ordinárias, a equação (1.41) tem uma solução $w$-periódica se, e somente se,

$$
\int_{0}^{w} u(t) \Psi_{0}(0) f(t) d t=0
$$

para toda solução $u w$-periódica da equação $\dot{u}(t)=-u(t) B$. Veja [4, pág 146].

Logo, para todo $u_{0}$ tal que $u_{0} e^{-B t}$ é $w$-periódica, devemos ter

$$
\int_{0}^{w} u_{0} e^{-B t} \Psi_{0}(0) f(t) d t=0
$$

Mas $u_{0} e^{-B t} \Psi_{0}(0)$ para esse conjunto de $u_{0}$ coincide com as soluções $w$-periódicas da equação adjunta (1.40).

Fica deste modo provada a primeira parte do teorema.

Para a segunda parte consideremos $V=\operatorname{col}\left(\psi_{1}, \ldots, \psi_{d}\right)$ uma base ortonormal relativamente ao produto interno de $\mathcal{L}^{2}([0, \infty))$ para as soluçốes $w$-periódicas da equação (1.40) e $V^{\prime}$ a transposta de $V$.

Definimos a aplicação $J: \mathcal{P}_{w} \rightarrow \mathcal{P}_{w}$ por

$$
J f=V^{\prime}\left[\int_{0}^{w} V(s) V^{\prime}(s) d s\right]^{-1} \int_{0}^{w} V(s) f(s) d s
$$

De acordo com a escolha de $V, J f=0$ se, e somente se, $\int_{0}^{w} V(s) f(s) d s=0$, ou seja, a equação (1.39) é satisfeita. Portanto, $f \in \mathcal{P}_{w}$ satisfaz (1.39) se, e somente se, $f \in(I-J) \mathcal{P}_{w}$.

Ainda, se (1.39) é satisfeita, pelo que vimos anteriormente, existe uma solução $x(f) w$-periódica da equação (1.30).

Consideramos então $K:(I-J) \mathcal{P}_{w} \rightarrow(I-\tilde{\pi}) \mathcal{P}_{w}$ definida como

$$
K f=(I-\tilde{\pi}) x(f)
$$

para toda $f \in(I-J) \mathcal{P}_{w}$.

$K$ é um operador linear contínuo e $K f$ satisfaz a equação (1.30) para toda $f \in(I-J) \mathcal{P}_{\boldsymbol{w}}$. 


\section{Capítulo 2}

\section{Bifurcação de Hopf}

Estaremos interessados neste capítulo, em estudar condições que sejam suficientes para garantir a existência de ramos locais de soluçũes periódicas não constantes, emanando de uma solução estacionária. Tais condições serão dadas no Teorema da Bifurcação de Hopf, apresentado na seção 2.3. Na seção 2.1, será feita uma breve explanação de alguns fatos básicos da teoria de bifurcação.Em 2.2, considerando uma família de $E D F R$ autônomas dependendo de um parâmetro real, extrairemos informações importantes, que nos serão úteis na demonstração do teorema de Hopf, que é um dos objetivos centrais deste trabalho. Essas informações serão obtidas a partir da análise da equação lineárizada.

\subsection{Fatos Básicos da Teoria Geral}

A teoria de bifurcação pode ser vista como um dos ramos da análise não linear, que estuda o comportamento local das soluçóes de uma equação. A principal motivação para estudá-la vem de problemas físicos; algumas referências a este respeito são citadas em [12].

Daremos a seguir, uma definição de ponto de bifurcação, baseada em [2].

Sejam $\mathbf{X}, \mathbf{Y}$, espaços de Banach, $\Lambda$ um conjunto aberto em um espaço de Banach, que chamaremos de conjunto de parâmetros, e $M: \Lambda \times X \rightarrow Y$ uma 
aplicação continuamente diferenciável.

Consideremos a equação

$$
M(\alpha, x)=0
$$

para $\alpha \in \Lambda, x \in X$.

Definimos uma solução da equação (2.1) como sendo um par $(\alpha, x) \in \Lambda \times X$, que satisfaz a equação (2.1).

Denotamos por $S \subset \Lambda \times X$ o conjunto de todas as soluçôes da equação (2.1) e, para cada $\alpha \in \Lambda$, consideramos

$$
S_{\alpha}=\{x \in X:(\alpha, x) \in S\}
$$

Dado um subconjunto aberto $\mathbf{U}$ de $\mathbf{X}$, dizemos que $S_{\alpha}$ é equivalente a $S_{\beta}$ em $\mathbf{U}$, se $S_{\alpha} \cap \mathbf{U}$ for homeomorfo a $S_{\beta} \cap \mathbf{U}$.

Definição $2.1 \alpha_{0}$ é um ponto de bifurcação para (2.1) se, para qualquer vizinhança $\mathrm{V}$ de $\alpha_{0}$, existir $x_{0} \in S_{\alpha_{0}}$, uma vizinhança $\mathrm{U}$ de $x_{0}$ e $\alpha_{1}, \alpha_{2}$ em $\mathrm{V}$, tais que $S_{\alpha_{1}}$ não é equivalente a $S_{\alpha_{2}}$ em $\mathrm{U}$.

Em particular, se $\alpha_{0}$ é ponto de bifurcação para (2.1), existe um $x_{0} \in S_{\alpha_{0}}$, tal que, para toda vizinhança $U$ de $\left(\alpha_{0}, x_{0}\right)$, existem pelo menos duas soluções distintas da equação $(2.1),\left(\alpha, x_{1}\right),\left(\alpha, x_{2}\right) \in \mathrm{U}$, para algum $\alpha \in \Lambda$.

Basicamente, o que se espera ao estudar teoria de bifurcação, é obter uma caracterizasaão dos elementos de $S_{\alpha}$, para $\alpha$ numa vizinhança de um ponto de bifurcação $\alpha_{0}$. Para esse fim, a parte linear de $M$ tem um papel fundamental. Com efeito, se a derivada de Frechét de $M$ com relação a $x$ em $\left(\alpha_{0}, x_{0}\right)$ for não singular, pelo teorema da função implícita, existe uma única função $x=x^{*}(\alpha)$ continuamente diferenciável numa vizinhança de $\alpha_{0}$, tal que $M\left(\alpha, x^{*}(\alpha)\right)=0$ e $x^{*}\left(\alpha_{0}\right)=x_{0}$. Resta, portanto, analisar os pontos para os quais $\frac{\partial}{\partial x} M\left(\alpha_{0}, x_{0}\right)$ é singular.

Suponhamos que $x_{0}$ seja solução da equação (2.1) para todo $\alpha$, isto é,

$$
M\left(\alpha, x_{0}\right)=0 .
$$

Se a equação (2.1) representa uma familia de $E D F R$, ou seja,

$$
M(\alpha, x)=\dot{x}(t)-F\left(\alpha, x_{t}\right),
$$


e estivermos interessados em sua soluções $w$-periódicas, então $M(\alpha,$.$) pode ser$ entendido como um operador em $P_{w}$ (mantendo a notação do capítulo anterior). Neste caso, denotando por $M_{x}\left(\alpha, x_{0}\right)$ a dcrivada de $M(\alpha, x)$ com relação a $x$ no punito $\left(\alpha, x_{0}\right)$, sabemos da teoria desenvolvida no capitulo 1 , que uma condição suficiente para estabilidade das soluções da equação (2.1), é

$$
\sigma\left(M_{x}\left(\alpha, x_{0}\right)\right) \subset\{z: \Re z<0\}
$$

Vamos assumir ainda, que isso ocorra para valores do parâmetro $\alpha$ menores que um certo $\alpha_{0} \in$, que para $\alpha>\alpha_{0}$,

$$
\sigma\left(M_{x}\left(\alpha, x_{0}\right) \cap\{z: \Re z>0\} \neq \emptyset\right.
$$

de forma que para esses valores de $\alpha$ temos instabilidade das soluções de (2.1). Sendo assim, em $\alpha=\alpha_{0}$, uma parte do espectro de $M_{x}\left(\alpha, x_{0}\right)$ deve cruzar o eixo imaginário, ocorrendo nesse ponto, mudanças sensíveis no comportamento das soluções de (2.1).

A bifurcação de Hopf se refere ao aparecimento de órbitas periódicas a partir de um equilíbrio estável, quando um parâmetro cruza um valor crítico. Quando isso ocorre, um par de auto valores conjugados deve cruzar o eixo imaginário.

Nas próximas seçôes deste capítulo, desenvolveremos com detalhes estas idéias.

\subsection{A aproximação linear e os auto valores}

Consideremos uma família de $E D F R$ autônomas, dependendo de um parâmetro real, da forma

$$
\dot{x}(t)=F\left(\alpha, x_{t}\right)
$$

onde $F(\alpha, \phi)$ tem primeira e segunda derivadas contínuas em $\alpha, \phi$, para $\alpha \in \mathbf{R}$ e $\phi \in \mathcal{C}$.

Assumiremos que (2.2) possui uma família $\phi=\phi(\alpha)$ de pontos de equilibrio, isto é, $F(\alpha, \phi(\alpha))=0$. Sem perda de generalidade, podemos supor que esta família é dada por $\phi \equiv 0$, ou seja, $F(\alpha, 0)=0$ para todo $\alpha \in \mathbf{R}$.

Denotando por $F_{\phi}(\alpha, 0)$ a derivada de $F(\alpha, \phi)$ com relação a $\phi$, calculada em $\phi=0$, definimos $L: \mathbf{R} \times \mathcal{C} \rightarrow \mathbf{R}^{\mathbf{n}}$ por

$$
L(\alpha) \psi=F_{\phi}(\alpha, 0) \psi
$$


e consideramos $f: \mathbf{R} \times \mathcal{C} \rightarrow \mathbf{R}^{\mathbf{n}}$ dada por

$$
f(\alpha, \phi)=F(\alpha, \phi)-L(\alpha) \phi
$$

para $(\alpha, \phi) \in \mathbf{R} \times \mathcal{C}$, tal que $f(\alpha, \phi)=o(|\phi|)$

Acrescentamos ainda as seguintes hipóteses:

$\left(H_{1}\right)$ Para um certo valor do parâmetro $\alpha$, digamos, $\alpha=0$; a $E D F R(L(0))$ tem duas raízes características imaginárias, $\lambda_{0}=i \nu_{0} \neq 0$ e $\bar{\lambda}_{0}=-i \nu_{0}$, que são simples e nenhuma outra raiz característica $\lambda_{j}$ é múltiplo inteiro de $\lambda_{0}$.

Como $\left\{L(\alpha): \mathcal{C} \rightarrow \mathbf{R}^{\mathbf{n}} ; \alpha \in \mathbf{R}\right\}$ é uma família de operadores lineares limitados continuamente diferenciáveis em $\alpha$, o lema 1.4 do capitulo 1 garante a existência de $\alpha_{0}>0$ e um auto valor simples $\lambda(\alpha)$ da $\Sigma D F R(L(\alpha))$, que tem derivada contínua $\lambda^{\prime}(\alpha)$ em $\alpha$, para $|\alpha|<\alpha_{0}$.

$$
\left(H_{2}\right) \Re \lambda^{\prime}(0) \neq 0 .
$$

Mostraremos na próxima seção que as hipóteses $\left(H_{1}\right)$ e $\left(H_{2}\right)$ implicam na existência de soluções periódicas não constantes da equação (2.2), para $\alpha$ pequeno, com período próximo a $2 \pi / \nu_{0}$.

Tomando $\alpha_{0}$ suficientemente pequeno, podemos assumir que $\Im \lambda(\alpha) \neq 0$ para $|\alpha|<\alpha_{0}$.

A cada auto valor $\lambda(\alpha)$, associamos a função $\phi_{\alpha} \in \mathcal{C}$ dada por $\phi_{\alpha}(t)=c e^{\lambda(\alpha) t}$, onde $c$ é constante para cada $\alpha$. Para $|\alpha|<\alpha_{0}, \phi_{\alpha} \in \mathcal{C}$ é base para o auto espaço generalizado da $\operatorname{EDFR}(L(\alpha))$ correspondente a $\lambda(\alpha)$.

Tomando $\Lambda=\{\lambda(\alpha), \bar{\lambda}(\alpha)\}$, efetuamos a decomposição de $\mathcal{C}$ por $\Lambda$, no sentido descrito no capítulo 1 .

Os elementos do auto espaço generalizado associado a $\Lambda$, sãu combinações lineares dos elementos dos auto espaços relativos a $\lambda(\alpha)$ e $\bar{\lambda}(\alpha)$. Sendo $\phi_{\alpha}$ e $\bar{\phi}_{\alpha}$ auto funções relativas a $\lambda$ e $\bar{\lambda}$ respectivamente e, desde que

$$
\Re \phi_{\alpha}=\frac{\phi_{\alpha}(t)+\bar{\phi}_{\alpha}(t)}{2} \text { e } \Im \phi_{\alpha}=\frac{\phi_{\alpha}(t)-\bar{\phi}_{\alpha}(t)}{2 i},
$$

as funções $\boldsymbol{\Phi}_{\alpha}=\left(\Re \phi_{\alpha}, \Im \phi_{\alpha}\right)$. formam uma base real para o auto espaço generalizado da $\operatorname{EDF} R(L(\alpha))$ associado a $\Lambda$.

Analogamente, obtemos uma base $\Psi_{\alpha}$ para o auto espaço da equação adjunta formal, $\operatorname{com}\left(\Psi_{\alpha}, \Phi_{\alpha}\right)=I$. 
Consideremos $\mathcal{C}=P_{\alpha} \oplus Q_{\alpha}$, onde $\Phi_{\alpha}$ é base para $P_{\alpha}$.

De acordo com o teorema 1.5 e a observação 1.2 do capítulo 1 , existe uma matriz $B(\alpha), 2 \times 2$, continuamente diferenciável, tal que

$$
\Phi_{\alpha}(\theta)=\Phi_{\alpha}(0) e^{B(\alpha) \theta},-r \leq \theta \leq 0,
$$

e os únicos auto valores de $B(\alpha)$ são $\lambda(\alpha)$ e $\bar{\lambda}(\alpha)$.

Por uma mudança de coordenadas é possível expressar $B(\alpha)$ na forma

$$
\left[\begin{array}{rr}
\mu(\alpha) & -\nu(\alpha) \\
\nu(\alpha) & \mu(\alpha)
\end{array}\right]
$$

onde $\mu(\alpha)=\Re \lambda(\alpha)$ e $\nu(\alpha)=\Im \lambda(\alpha)$.

Como $\mu(\alpha)$ é continuamente diferenciável para $|\alpha|<\alpha_{0}$ e $\mu^{\prime}(0) \neq 0$, pelo Teorema da Função Inversa, existe uma vizinhança de zero, tal que $\mu$ restrita a essa vizinhança, tem inversa contínua e diferenciável. Restringindo $\alpha_{0}$ se necessário, para $|\alpha|<\alpha_{0}$ podemos redefinir o parâmetro $\alpha$, de modo que

$$
B(\alpha)=\left[\begin{array}{cc}
\alpha & -\nu\left(\mu^{-1}(\alpha)\right) \\
\nu\left(\mu^{-1}(\alpha)\right) & \alpha
\end{array}\right]
$$

Definindo

$$
\begin{aligned}
& \gamma(\alpha)= \begin{cases}\frac{-\left(\nu\left(\mu^{-1}(\alpha)\right)+\nu_{0}\right)}{\alpha} & \text { se } \alpha \neq 0 \\
\lim _{\alpha \rightarrow 0} \frac{-\left(\nu\left(\mu^{-1}(\alpha)\right)+\nu_{0}\right)}{\alpha} & \text { se } \alpha=0\end{cases} \\
& B_{0}=\left[\begin{array}{rr}
0 & 1 \\
-1 & 0
\end{array}\right] \text { e } B_{1}(\alpha)=\left[\begin{array}{cc}
1 & \gamma(\alpha) \\
-\gamma(\alpha) & 1
\end{array}\right]
\end{aligned}
$$

obtemos ainda

$$
B(\alpha)=\nu_{0} B_{0}+\alpha B_{1}(\alpha)
$$

com $\gamma(\alpha)$ continuamente diferenciável para $0 \leq|\alpha|<\alpha_{0}$.

\subsection{Teorema da Bifurcação de Hopf}

Estabeleceremos nesta seção o Teorema da Bifurcação de Hopf para equações autônomas com retardamento e apresentaremos uma demonstração. 
As técnicas usadas serão essencialmente o Método de Liapunov - Schmidt, que apresentamos no Apêndice B, e o Teorema da Função Implícita.

Utilizaremos as notações introduzidas na seção anterior.

Teorema 2.1 (Bifurcação de Hopf). Se $F(\alpha, \phi)$ tem primeira e segunda derivadas contínuas com relação a $\alpha$ e $\phi, F(\alpha, 0)=0$, para todo $\alpha$, e as hipóteses $\left(H_{1}\right)$ $e\left(H_{2}\right)$ são satisfeitas, entâo existem constantes $a_{0}>0, \alpha_{0}>0, \delta_{0}>0$, funções reais $\alpha(a), w(a)$ e uma função $w(a)$-periódica, $x^{*}(a)$, todas continuamente diferenciáveis em a para $|a|<a_{0}$, tal que $x^{*}(a)$ é solução da equação (2.2) com

$$
x_{0}^{*}(a)^{P_{\alpha}}=\Phi_{\alpha(a)} y_{0}^{*}(a), \quad x_{0}^{*}(a)^{Q_{\alpha}}=z_{0}^{*}(a),
$$

onde $y_{0}^{*}(a)=\operatorname{col}(a, 0)+o(|a|)$ e $z_{0}^{*}(a)=o(|a|)$, quando $|a| \rightarrow 0$. Além disso, para $|\alpha|<\alpha_{0},\left|w-2 \pi / \nu_{0}\right|<\delta_{0}$ e toda solução da equação (2.2) com $\left|x_{t}\right|<\delta_{0}$ deve ser do tipo acima, exceto por defasagem.

\section{Demonstração:}

Sejam $\beta \in(-1,1], w_{0}=2 \pi / \nu_{0} \mathrm{e}$

$$
u(\tau)=x((1+\beta) \tau), \quad \tau \geq 0,
$$

onde $x$ é uma solução da equação (2.2).

Definimos $u_{\tau, \beta}$, como um elemento do espaço $\mathcal{C}\left([-r, 0], \mathbf{R}^{\mathbf{n}}\right)$, por

$$
u_{\tau, \beta}(\theta)=u\left(\tau+\frac{\theta}{1+\beta}\right),-r \leq \theta \leq 0 .
$$

Afim de facilitar a compreensão, dividiremos esta prova em passos.

10. Passo: A equação (2.2) é equivalente à equação

$$
\frac{d u(\tau)}{d \tau}=(1+\beta) F\left(\alpha, u_{\tau, \beta}\right)
$$

De fato, seja $t=(1+\beta) \tau$. Para todo $\theta \in[-r, 0]$,

$$
x_{t}(\theta)=x(t+\theta)=u\left(\tau+\frac{\theta}{1+\beta}\right)=u_{\tau, \beta}(\theta)
$$




$$
\begin{aligned}
& \text { Portanto, } x_{t}=u_{\tau, \beta} \mathrm{e} \\
& \qquad \begin{aligned}
\frac{d x(t)}{d t}=F\left(\alpha, x_{t}\right) & \Leftrightarrow \frac{d}{d \tau} x((1+\beta) \tau) \frac{1}{1+\beta}=F\left(\alpha, u_{\tau, \beta}\right) \\
& \Leftrightarrow \frac{d u(\tau)}{d \tau}=(1+\beta) F\left(\alpha, u_{\tau, \beta}\right) .
\end{aligned}
\end{aligned}
$$

2 9 Passo: Se a equação (2.7) tiver uma solução $w_{0}$-periódica, então a equação (2.2) tem uma solução $(1+\beta) w_{0}$-periódica e reciprocamente.

De fato, se $u(\tau)$ é solução de (2.7) tal que $u(\tau)=u\left(\tau+w_{0}\right)$, para todo $\tau \geq 0$, então $x(t)=u\left(\frac{t}{1+\beta}\right)$ é solução $(1+\beta) w_{0}$-periódica da equação (2.2).

Reciprocamente, se $x(t)$ é solução de (2.2) tal que $x(t)=x\left(t+(1+\beta) w_{0}\right)$, para todo $t \geq 0$, então $u(\tau)=x((1+\beta) \tau)$ é solução $w_{0}$-periódica de $(2.7)$.

Observamos que $w_{0}$ é um período constante, mas $(1+\beta) w_{0}$ varia conforme varia o parâmetro $\beta$. A razão dessa mudança na escala de tempo é, justamente, criar circunstância onde se tenha periodo constante.

39 Passo: A equação (2.7) pode ser reescrita como

$$
\frac{d u(\tau)}{d \tau}=L(0) u_{\tau}+N\left(\beta, \alpha, u_{\tau}, u_{\tau, \beta}\right)
$$

onde

$$
N\left(\beta, \alpha, u_{\tau}, u_{\tau, \beta}\right):=(1+\beta) L(\alpha) u_{\tau, \beta}-L(0) u_{\tau}+(1+\beta) f\left(\alpha, u_{\tau, \beta}\right) .
$$

Isto é óbvio, desde que por (2.4),

$$
F\left(\alpha, u_{\tau, \beta}\right)=L(\alpha) u_{\tau, \beta}+f\left(\alpha, u_{\tau, \beta}\right)
$$

Isto significa, que podemos considerar a equação (2.8) como uma perturbação da equação linear autônoma

$$
\frac{d u(\tau)}{d \tau}=L(0) u_{\tau}
$$

4. Passo: As colunas de $U(\tau):=\Phi_{0}(0) e^{B(0) \tau}, \tau \in \mathbf{R}$, formam uma base para as soluções $w_{0}$-periódicas da equação $(2.10)$, e as linhas de $V(\tau):=e^{-B(0) \tau} \Psi_{0}(0)$, 
formam uma base para as soluções $w_{0}$ - periódicas da equação adjunta formal associada à equação (2.10).

De fato, sabemos que as colunas de $U(\tau)$ formam uma base para as soluções da equação (2.10). Veremos que estas são sempre $w_{0}$-periódicas.

Com efeito, conforme vimos inicialmente,

$$
B(\alpha)=\nu_{0} B_{0}+\alpha B_{1}(\alpha),
$$

e portanto

$$
B(0)=\left[\begin{array}{cc}
0 & \nu_{0} \\
-\nu_{0} & 0
\end{array}\right]
$$

só possui auto valores imaginários puros, que são dados por $i \nu_{0} e-i \nu_{0}$.

Logo, as soluções de (2.10) serão combinações lineares de $\operatorname{sen} \nu_{0} \tau, \cos \nu_{0} \tau$ e portanto, $w_{0}$-periódicas.

Analogamente, as linhas de $V(\tau)$ formam uma base para as soluções $w_{0^{-}}$ periódicas da equação adjunta formal associada à equação (2.10).

Queremos determinar condições necessárias e suficientes para a existência de soluçôes $w_{0}$-periódicas da equação (2.8). Utilizaremos para isso a Alternativa de Fredholm (teorema 1.7 do capítulo 1). Esse teorema não pode ser aplicado diretamente à equação (2.8), desde que não se verifica a hipótese $N\left(\beta, \alpha, u_{\tau}, u_{\tau, \beta}\right) \in P_{w_{\circ}}$.

\section{Passo:}

Seja $v(\tau) \in P_{w_{0}}$.

$v(\tau)$ é uma soluçâo $w_{o}$-periódica da equação $(2.8)$ se, e somente se, $v(\tau)$ é solução $w_{0}$-periódica da equação

$$
\frac{d u(\tau)}{d \tau}=L(\hat{0}) u_{\tau}+N\left(\beta, \alpha, v_{\tau}, v_{\tau, \beta}\right)
$$

e $N\left(\beta, \alpha, v_{\tau}, v_{\tau, \beta}\right) \in P_{w_{0}}$.

Assim, podemos aplicar o teorema 1.7 à equação (2.11), obtendo:

- A equação (2.11) possui solução $w_{o}$-periódica se, e somente se, $\int_{0}^{w_{o}} y(\tau) N\left(\beta, \alpha, v_{\tau}, v_{\tau, \beta}\right) d \tau=0$, para toda solução $w_{\circ}$-periódica da adjunta formal $\dot{y}(s)=-\int_{-r}^{0} y(s-\theta) d \eta(\theta)$. 
- Existe uma projeção contínua $J: P_{w_{o}} \rightarrow P_{w_{o}}$ tal que

$$
\left\{f \in P_{w_{o}}: \int_{0}^{w_{0}} y(\tau) f\left(\beta, \alpha, v_{\tau}, v_{\tau, \beta}\right) d \tau=0\right\}=(I-J) P_{w_{0}} .
$$

- Existe um operador linear contínuo $K:(I-J) P_{w_{o}} \rightarrow(I-\tilde{\pi}) P_{w_{o}}$ tal que $K f$ é solução de $\dot{u}(\tau)=L(0) u_{\tau}+f\left(\beta, \alpha, v_{\tau}, v_{\tau, \beta}\right)$ para toda $f \in(I-J) P_{w_{o}}$, onde $\tilde{\pi}: P_{w_{o}} \rightarrow P_{w_{o}}$ é projeção contínua de $P_{w_{o}}$ sobre as soluções $w_{o}$-periódicas de $(2.10)$.

Seja $A: \mathcal{D}(A) \subset P_{w_{0}} \rightarrow P_{w_{o}}$ o operador linear definido por

$$
A(\phi)=\dot{\phi}(0)-L(0) \phi \text {. }
$$

Reescrevemos (2.11) como

$$
A\left(u_{\tau}\right)=N\left(\beta, \alpha, v_{\tau}, v_{\tau, \beta}\right) .
$$

Estudaremos esta equação através do Método de Liapunov-Schmidt.

6? Passo: $\mathcal{N}(A)=\tilde{\pi} P_{w_{0}}$ e $\mathcal{R}(A)=(I-J) P_{w_{0}}$.

Facilmente vemos que $\mathcal{N}(A)=\tilde{\pi} P_{w_{0}}$.

$$
\mathcal{R}(A)=\left\{f \in P_{w_{o}}: \text { existe solução para } A\left(u_{\tau}\right)=f\left(\beta, \alpha, v_{\tau}, v_{\tau, \beta}\right)\right\}
$$

$f\left(\beta, \alpha, v_{\tau}, v_{\tau, \beta}\right) \in(I-J) P_{w_{o}}$ se, e somente se, $\int_{0}^{w_{0}} y(\tau) f\left(\beta, \alpha, v_{\tau}, v_{\tau, \beta}\right) d \tau=0$, para toda solução $y w_{o}$-periódica da adjunta formal.

Pelo teorema 1.7 , isto é equivalente a dizer que existe solução $w_{o}$-periódica para (2.11), ou seja, $f \in \mathcal{R}(A)$.

79 Passo: $K$ satisfaz

(i) $A K=I \mathrm{em} \mathcal{R}(A) \mathrm{e}$

(ii) $K A=I-\tilde{\pi} \operatorname{em} \mathcal{D}(A)$.

De fato:

(i) Seja $f \in \mathcal{R}(A)=(I-J) P_{w_{0}}$. 
$K f$ é solução de $\dot{u}(\tau)=L(0) u_{\tau}+f\left(\beta, \alpha, v_{\tau}, v_{\tau, \beta}\right)$, então

$$
A K f=\frac{d}{d \tau}(K f)(0)-L(0) K f=f
$$

Portanto, $A K=I$ em $\mathcal{R}(A)$.

(ii) Seja $\phi \in \mathcal{D}(A) \subset P_{w_{0}}$.

$\phi=\phi_{1}+\phi_{2}, \operatorname{com} \phi_{1} \in \tilde{\pi} P_{w_{o}}$ e $\phi_{2} \in(I-\tilde{\pi}) P_{w_{o}}$.

Então, como $\tilde{\pi} P_{w_{o}}=\mathcal{N}(A)$,

$$
K A \phi=K A\left(\phi_{1}+\phi_{2}\right)=K A \phi_{1}+K A \phi_{2}=K A \phi_{2}=\phi_{2}=(I-\tilde{\pi}) \phi .
$$

Portanto, $K A=I-\tilde{\pi}$ em $P_{w_{o}}$.

Logo, a equação (2.11) é equivalente ao sistema

$$
\left\{\begin{array}{l}
u_{\tau}-\tilde{\pi}\left(u_{\tau}\right)=K(I-J) N\left(\beta, \alpha, v_{\tau}, v_{\tau, \beta}\right) \\
J N\left(\beta, \alpha, v_{\tau}, v_{\tau, \beta}\right)=0
\end{array}\right.
$$

89. Passo: $\tilde{\pi}\left(u_{\tau}\right)=U(\tau) \operatorname{col}(a, 0)$

De fato, $\tilde{\pi}\left(u_{\tau}\right)$ pertence ao conjunto das soluções $w_{0}$-periódicas de (2.10). Como as colunas de $U(\tau)=\Phi_{0}(0) e^{B(0) \tau}$ formam uma base para as soluções $w_{0^{-}}$ periódicas de (2.10),

$$
\tilde{\pi}\left(u_{\tau}\right)=U(\tau) \operatorname{col}(x, y)
$$

As colunas de $U(\tau+h)=\Phi_{0}(0) e^{B(0)(\tau+h)}$ também constituem uma base para as soluções $w_{0}$-periódicas de $(2.10)$, pois

$$
U(\tau+h)=\Phi_{0}(0) e^{B(0) h} e^{B(0) \tau}=\xi_{0}(0) e^{B(0) \tau},
$$

onde $\xi_{0}(0)=\Phi_{0}(0) e^{B(0) h}$.

Mostraremos que existem $a$ e $h$ tais que

$$
U(\tau+h) \operatorname{col}(x, y)=U(\tau) \operatorname{col}(a, 0)
$$

Sendo

$$
U(\tau+h) \operatorname{col}(x, y)=U(\tau) e^{B(0) h} \operatorname{col}(x, y)
$$


basta mostrar que existem $a$ e $h$ tais que

$$
e^{B(0) h} \operatorname{col}(x, y)=\operatorname{col}(a, 0) .
$$

Temos

$$
e^{B(0) h} \operatorname{col}(x, y)=\left[\begin{array}{ll}
e^{i \nu_{0} h} & 0 \\
0 & e^{-i \nu_{0} h}
\end{array}\right]\left[\begin{array}{l}
x \\
y
\end{array}\right]
$$

e utilizando a forma canônica real,

$$
\begin{aligned}
e^{B(0) h} \operatorname{col}(x, y) & =\left[\begin{array}{cc}
\cos \nu_{0} h & \operatorname{sen} \nu_{0} h \\
-\operatorname{sen} \nu_{0} h & \cos \nu_{0} h
\end{array}\right]\left[\begin{array}{l}
x \\
y
\end{array}\right] \\
& =\left[\begin{array}{c}
x \cos \nu_{0} h+y \operatorname{sen} \nu_{0} h \\
-x \operatorname{sen} \nu_{0} h+y \cos \nu_{0} h
\end{array}\right]
\end{aligned}
$$

Portanto, o problema se reduz a mostrar a existência de soluções $h$ de

$$
-x \operatorname{sen} \nu_{o} h+y \cos \nu_{0} h=0,
$$

o que é um fato óbvio.

Logo, podemos expressar $\tilde{\pi}\left(u_{\tau}\right)$ como $U(\tau) \operatorname{col}(a, 0)$.

Como a equação (2.11) é equivalente ao sistema (2.13), este resultado implica que toda solução $w_{e}$-periódica da equação (2.11), exceto por defasagem, é solução das equações

$$
\begin{gathered}
u_{\tau}=U(\tau) \operatorname{col}(a, 0)+K(I-J) N\left(\beta, \alpha, v_{\tau}, v_{\tau, \beta}\right) \\
J N\left(\beta, \alpha, v_{\tau}, v_{\tau, \beta}\right)=0
\end{gathered}
$$

e reciprocamente.

90 Passo: Resolução de (2.14):

Escrevemos a equação (2.14) como

$$
A u_{\tau}=A U(\tau) \operatorname{col}(a, 0)+A K(I-J) N\left(\beta, \alpha, v_{\tau}, v_{\tau, \beta}\right) .
$$
que

Como todo elemento de $\tilde{\pi} P_{w_{o}}$ é da forma $U(\tau) \operatorname{col}(a, 0)$ e $\tilde{\pi} P_{w_{0}}=\mathcal{N}(A)$, segue

$$
A U(\tau) \operatorname{col}(a, 0)=0 .
$$

Sabemos também que $A K=I$ em $\mathcal{R}(A)$. Então, $A u_{\tau}=(I-J) N\left(\beta, \alpha, v_{\tau} v_{\tau, \beta}\right)$. 
$A$ restrito a $(I-J) P_{w_{0}}$ é um isomorfismo, pois $(I-J) P_{w_{o}}=\mathcal{R}(A)$, e é contínuo juntamente com sua primeira derivada.

Logo, podemos resolver a equação (2.14) via Teorema da Função Implícita. Obtemos uma solução $u^{*}=u^{*}(a, \hat{\beta}, \alpha)$ para $a, \beta, \alpha$, numa vizinhança suficientemente pequena de zero, continuamente diferenciável em $a, \beta, \alpha$, satisfazendo

$$
u^{*}(a, \beta, \alpha)-U(.) \operatorname{col}(a, 0)=o(|a|)
$$

quando $|a| \rightarrow 0$, para $\alpha, \beta$ suficientemente próximos de zero.

Como $u^{*}(\tau)=u^{*}(a, \beta, \alpha)(\tau)$ satisfaz a equaçâo (2.14), também satisfaz a equação integral associada a (2.7); logo, é continuamente diferenciável em $\tau$.

Portanto, toda soluçâu $w_{0}$-periódica da equação (2.11) é obtida encontrandose soluções $a, \beta, \alpha$ da equação de bifurcação

$$
J N\left(\beta, \alpha, u_{.}^{*}(a, \beta, \alpha), u_{., \beta}^{*}(a, \beta, \alpha)\right)=0 .
$$

Usando a definição de $J$ dada pela relação (1.42) do capítulo 1, ou seja,

$$
J f=V^{\prime}\left[\int_{0}^{w_{0}} V(s) V^{\prime}(s) d s\right]^{-1} \int_{0}^{w_{0}} V(s) f(s) d s,
$$

$f \in P_{w_{0}}$, a equação (2.16) torna-se equivalente à equação

$$
G(a, \beta, \alpha):=\int_{0}^{w_{o}} e^{-B(0) s} \Psi_{0}(0) N\left(\beta, \alpha, u_{s}^{*}(a, \beta, \alpha), u_{s, \beta}^{*}(a, \beta, \alpha)\right) d s=0 .
$$

Assim, basta resolver a equação

$$
G(a, \beta, \alpha)=0
$$

para todo $\beta \in \alpha$.

Como $G(0, \beta, \alpha)=0$ para todo $\beta, \alpha$, definimos

$$
\begin{aligned}
& H(a, \beta, \alpha)=\frac{G(a, \beta, \alpha)}{a} \text { para } a \neq 0 \\
& H(0, \beta, \alpha)=\lim _{a \rightarrow 0} \frac{G(a, \beta, \alpha)}{a}
\end{aligned}
$$

e resolvemos $H(a, \beta, \alpha)=0$.

10 Passo: $H(0,0, \alpha)=\int_{0}^{w_{0}} e^{-B(0) s} \Psi_{0}(0)[L(\alpha)-L(0)] U_{\iota} e_{1} d s$, onde $e_{1}=\operatorname{col}(1,0)$. 
De fato, como $u^{*}(a, \beta, \alpha)=U(.) \operatorname{col}(a, 0)$ numa vizinhança suficientemente pequena de zero,

$$
\left.\frac{\partial}{\partial a} u^{*}(a, \beta, \alpha)\right|_{a=0}=U(.) \operatorname{col}(1,0)
$$

e como $\left.\frac{\partial}{\partial a} f\left(\alpha, u_{\theta}^{*}(a, 0, \alpha)\right)\right|_{a=0}=0$ para todo $\alpha$, segue que

$$
\begin{aligned}
H(0,0, \alpha) & =\left.\frac{\partial}{\partial a} G(a, 0, \alpha)\right|_{a=0} \\
& =\frac{\partial}{\partial a}\left[\int_{0}^{w_{o}} e^{-B(0) s} \Psi_{0}(0) N\left(0, \alpha, u_{s}^{*}(a, 0, \alpha), u_{s}^{*}(a, 0, \alpha) d s\right]_{a=0}\right. \\
& =\frac{\partial}{\partial a}\left[\int_{0}^{w_{0}} e^{-B(0) s} \Psi_{0}(0)\left(L(\alpha) u_{s}^{*}(a, o, \alpha)-L(0) u_{s}^{*}(a, o, \alpha)\right) d s\right]_{a=0} \\
& =\int_{0}^{w_{o}} e^{-B(0) s} \Psi_{0}(0)(L(\alpha)-L(0)) U_{s} e_{1} d s .
\end{aligned}
$$

119. Passo: A derivada de $H(a, \beta, \alpha)$ com relação a $\alpha$ no ponto $(0,0,0)$ é

$$
w_{O}\left[\begin{array}{c}
1 \\
-\gamma(0)
\end{array}\right]
$$

De fato:

$$
\begin{aligned}
\frac{\partial}{\partial \alpha} H(0,0, \alpha) & =\frac{\partial}{\partial \alpha}\left[\int_{0}^{w_{0}} e^{-B(0) s_{0}} \Psi_{0}(0)(L(\alpha)-L(0)) U_{s} e_{1} d s\right] \\
& =\int_{0}^{w_{0}} \frac{\partial}{\partial \alpha}\left[e^{-B(0) s} \Psi_{0}(0)(L(\alpha)-L(0)) U_{s} e_{1}\right] d s \\
& =\int_{0}^{w_{o}} e^{-B(0) \diamond} \Psi_{0}(0) L^{\prime}(\alpha) U_{s} e_{1} d s \\
& =\int_{0}^{w_{0}} e^{-B(0) s} \Psi_{0}(0) L^{\prime}(\alpha) \Phi_{0}(0) e^{B(0)} e^{B(0) s} e_{1} d s
\end{aligned}
$$

Pela observação 1.2 do capitulo 1 ,

$$
B^{\prime}(\alpha)=\Psi_{\alpha}(0) L^{\prime}(\alpha) \Phi_{\alpha}(0) e^{B(\alpha)}
$$

para todo $\alpha \in \mathbf{R}$.

Logo,

$$
\left.\frac{\partial}{\partial \alpha} H(0,0, \alpha)\right|_{\alpha=0}=\int_{0}^{w_{0}} e^{-B(0) s} B^{\prime}(0) e^{B(0) s} e_{1} d s=\int_{0}^{w_{0}} B^{\prime}(0) e_{1} d s,
$$

$\operatorname{com}$

$$
B^{\prime}(0)=\left[\begin{array}{cc}
1 & \gamma(0) \\
-\gamma(0) & 1
\end{array}\right]
$$


Portanto,

$$
\left.\frac{\partial}{\partial \alpha} H(0,0, \alpha)\right|_{\alpha=0}=\left[\begin{array}{c}
1 \\
-\gamma(0)
\end{array}\right] \int_{0}^{w_{0}} d s=w_{0}\left[\begin{array}{c}
1 \\
-\gamma(0)
\end{array}\right]
$$

12. Passo: $\quad H(0, \beta, 0)=\int_{0}^{w_{o}} e^{-B(0) s} \Psi_{0}(0)\left[(1+\beta) L(0) U_{s, \beta} e_{1}-L(0) U_{o} e_{1}\right] d s$

De fato, analogamente ao que foi feito no $10^{\circ}$ Passo, temos

$$
\begin{aligned}
H(0, \beta, 0)= & \left.\frac{\partial}{\partial a} G(a, \beta, 0)\right|_{a=0} \\
= & \frac{\partial}{\partial a}\left[\int_{0}^{w_{o}} e^{-B(0) s} \Psi_{0}(0)(1+\beta) L(0) u_{s, \beta}^{*}(a, \beta, 0) d s\right]_{a=0} \\
& -\frac{\partial}{\partial a}\left[\int_{0}^{w_{0}} e^{-B(0) s} \Psi_{0}(0) L(0) u_{s}^{*}(a, \beta, 0) d s\right]_{a=0} \\
& +\frac{\partial}{\partial a}\left[e^{-B(0) s} \Psi_{0}(0)(1+\beta) f\left(0, u_{s, \beta}^{*}(a, \beta, 0)\right) d s\right]_{a=o} \\
= & \int_{0}^{w_{0}} e^{-B(0) s} \Psi_{0}(0)\left[(1+\beta) L(0) U_{s, \beta} e_{1}-L(0) U_{s} e_{1}\right] d s
\end{aligned}
$$

13ㅇ Passo:

$$
\frac{d U(s /(1+\beta))}{d(s /(1+\beta))}=L(\overline{0}) U_{\diamond /(1+\beta), \beta}
$$

De fato, sabemos que as colunas de $U(s)$ formam uma base para as soluções $u_{\circ}$-periódicas de $(2.10)$ e portanlo,

$$
\frac{d U(s)}{d s}=L(0) U_{s}
$$

Através da mudança de variável $s \leftrightarrow s /(1+\beta)$, obtemos o resultado.

Escrevendo a integral que expressa $H(0, \beta, 0)$, no $12^{\circ}$ Passo, como diferença de duas integrais e mudando a variável $s$ para $s /(1+\beta)$ na primeira integral, vemos que

$$
H(0, \beta, 0)=\beta \int_{0}^{w_{0}} e^{-B(0) s} \Psi_{0}(0) \Phi_{0}(0) e^{B(0) s} B(0) e_{1} d s
$$

149. Passo: $H(0, \beta, 0)=\beta w_{0} B(0) e_{1}$ 
De fato, sabemos do capítulo 1 que se $x$ é solução de (2.10) e $y$ é solução da equação adjunta de (2.10), então, $\left(y^{t}, x_{t}\right)$ é constante, para todo $t$.

Portanto, no nosso caso, temos

$$
\begin{aligned}
I= & \left(e^{-B(0)(s+.)} \Psi_{0}(0), \Phi_{0}(0) e^{B(0)(s+\cdot)}\right) \\
= & e^{-B(0) s} \Psi_{0}(0) \Phi_{0}(0) e^{B(0) s} \\
& -\int_{-r}^{0} \int_{0}^{\theta} e^{-B(0)(s+\xi-\theta)} \Psi_{0}(0) d \eta(\theta) \Phi_{0}(0) e^{B(0)(s+\xi)} d \xi
\end{aligned}
$$

para todo $s \in \mathbf{R}$.

Integrando ambos os membros de zero a $w_{o}$ e usando o fato que a segunda integral do segundo membro é zero, vem que

$$
w_{0} I=\int_{0}^{w_{c}} e^{-B(0) s} \Psi_{0}(0) \Phi_{0}(0) e^{B(0) s} d s
$$

Multiplicando ambos os membros dessa igualdade por $\beta B(0) e_{1}$ à direita, obtemos

$$
\begin{aligned}
\beta w_{o} B(0) e_{1} & =\beta \int_{0}^{w_{0}} e^{-B(0) s} \Psi_{0}(0) \Phi_{0}(0) e^{B(0) s} B(0) e_{1} d s \\
& =H(0, \beta, 0) .
\end{aligned}
$$

Segue, portanto, que $H(0,0,0)=0 \mathrm{e}$

$$
\begin{aligned}
& \frac{d}{d(\beta, \alpha)} H(0,0,0)=\left(w_{o} B(0) e_{1} w_{o} B_{1}(0) \epsilon_{1}\right) \\
& =w_{0}\left[\begin{array}{cc}
0 & 1 \\
-\nu_{0} & -\gamma(0)
\end{array}\right]
\end{aligned}
$$

Então, $\operatorname{det}\left(\frac{d}{d(\beta, \alpha)} H(0,0,0)\right) \neq 0$.

Aplicando novamente o Teorema da Função Implícita, obtemos funções $\beta(a)$ e $\alpha(a)$ continuamente diferenciáveis, tais que $\beta(0)=\alpha(0)=0$, com

$$
H(a, \beta(a), \alpha(a))=0 .
$$

Esta soluçâo é única para $a$ numa vizinhança do zero, digamos, para $|a|<a_{0}$. 
Portanto, $G(a, \beta(a), \alpha(a))=0$ e concluimos assim, que $u^{*}(a, \beta(a), \alpha(a))$ é solução $w_{0}$-periódica da equação (2.8).

$x^{*}(a)$, correspondente a $u^{*}(a)$, será solução da equação $(2.2), w(a)$-periódica, onde $w(a)=(1+\beta(a)) w_{0}$.

Mostraremos a seguir, que $x_{0}^{*}(a)^{P_{a}}$ e $x_{0}^{*}(a)^{Q_{a}}$ são dados pelà equação (2.5), quando $|a| \rightarrow 0$.

159 Passo: $x_{0}^{*}(a)^{P_{\alpha(a)}}=\Phi_{\alpha(a)} y_{0}^{*}(a), y_{0}^{*}(a)=\operatorname{col}(a, 0)+o(|a|), \quad|a| \rightarrow 0$.

De fato, se $x_{0}^{*}(a)^{P_{a(a)}}=\Phi_{\alpha(a)} y_{0}^{*}(a)$, então

$$
\lim _{|a| \rightarrow 0} \frac{x_{0}^{*}(a)^{P_{\alpha(a)}}}{|a|}=\Phi_{\alpha(0)} \lim _{|a| \rightarrow 0} \frac{y_{0}^{*}(a)}{|a|}=U \lim _{|a| \rightarrow 0} \frac{y_{0}^{*}(a)}{|a|}
$$

Por outro lado, como

$$
\begin{gathered}
x_{0}^{*}(a)(\theta)=u_{0, \beta(a)}^{*}(a, \beta(a), \alpha(a))(\theta), \quad-r \leq \theta \leq 0, \\
\lim _{|a| \rightarrow 0} \frac{x_{0}^{*}(a)^{P_{\alpha(a)}}}{|a|}=\lim _{|a| \rightarrow 0} \frac{u_{0, \beta(a)}^{*}(a, \beta(a), \alpha(a))^{P_{\alpha(a)}}}{|a|}=U e_{1} .
\end{gathered}
$$

Portanto,

$$
\begin{aligned}
U \lim _{|a| \rightarrow 0} \frac{y_{0}^{*}(a)}{|a|}=U e_{1} & \Rightarrow U\left(\lim _{|a| \rightarrow 0} \frac{y_{0}^{*}(a)}{|a|}-e_{1}\right)=0 \\
& \Rightarrow \lim _{\mid u i \rightarrow 0} \frac{y_{0}^{*}(a)-\operatorname{col}(a, 0)}{|a|}=0
\end{aligned}
$$

ou seja,

$$
y_{0}^{*}(a)=\operatorname{col}(a, 0)+o(|a|),
$$

quando $|a| \rightarrow 0$.

16 Passo: $x_{0}^{*}(a)^{Q_{\alpha(a)}}=z_{0}^{*}(a), \quad z_{0}^{*}(a)=o(|a|), \quad|a| \rightarrow 0$.

De fato, se $x_{0}^{*}(a)^{Q_{a(a)}}=z_{0}^{*}(a)$, então, como

$$
\begin{gathered}
\lim _{|a| \rightarrow 0} \frac{x_{0}^{*}(a)^{Q_{\alpha(a)}}}{|a|}=\lim _{|a| \rightarrow 0} \frac{u_{0, \beta(a)}^{*}(a, \beta(a), \alpha(a))^{Q_{a(a)}}}{|a|}=0, \\
\quad z_{0}^{*}(a)=o(|a|),
\end{gathered}
$$


quando $|a| \rightarrow 0$.

Finalmente, a unicidade das funções $\alpha(a), \beta(a)$, para $|a|<a_{0}$, e a hipótese dos auto valores da $\operatorname{EDFR}(L(0))$ não serem múltiplos inteiros de $\lambda_{0}$, implicam que, se $x$ for solução $w$-periódica da equação $(2.2)$, com $\left|w-w_{0}\right|<\delta_{0},|\alpha|<\alpha_{0}$ e $\left|x_{t}\right|<\delta_{0}$, então, $x$ será do tipo mencionado acima, exceto por defasagem.

Com isto, completamos a prova da teorema.

Observação 2.1: Queremos enfatizar a dependência do período $w$, da raiz imaginária $\lambda_{0}=i \nu_{0}$, no caso de estarem satisfeitas as condições impostas no teorema 2.1. Notamos que se $\nu_{0}$ for muito pequeno, o período torna-se grande, ou mais especificamente, maior que $2 \pi$; se $\nu_{0}$ for grande, o período torna-se menor, isto é, a oscilação é mais rápida.

Referimo-nos as conclusões do teorema 2.1 como sendo uma Bifurcação de Hopf. 


\section{Capítulo 3}

\section{Uma aplicação a equações diferenciais diferença no plano}

Aplicaremos o teorema 2.1 do capítulo anterior à equação diferencial retardada

$$
\dot{x}(t)=\alpha f(x(t), x(t-1))
$$

onde $x=\operatorname{col}\left(x_{1}, x_{2}\right), f=\operatorname{col}\left(f_{1}, f_{2}\right)$ é uma função de classe $C^{3}$ de $\mathbf{R}^{4}$ em $\mathbf{R}^{2}$ e $\alpha$ é um parâmetro real.

Verificaremos, sob condições adequadas, a existência de uma sequência de valores do parâmetro $\alpha$ onde ocorre uma bifurcação de Hopf. Isto será feito a partir de uma descrição da localização dos auto valores da equação linearizada de (3.1)

Para este estudo baseamo-nos em [13].

\subsection{Considerações Iniciais}

Se $y(t)=x(t-1)$, reescrevemos (3.1) como

$$
\dot{x}(t)=\alpha f(x(t), y(t)), \quad y=\operatorname{col}\left(y_{1}, y_{2}\right)
$$

Sejam $\delta_{1}=\frac{\partial f_{1}(0)}{\partial y_{2}} e^{-}-\delta_{2}=\frac{\partial f_{2}(0)}{\partial y_{1}}$ 
Estabeleceremos as seguintes hipóteses:

$\left(H_{1}\right) y_{2} f_{1}(0, y)>0, \quad y_{2} \neq 0$ e $y_{1} f_{2}(0, y)<0, \quad y_{1} \neq 0$.

$\left(H_{2}\right) \delta_{1}, \delta_{2}>0$.

$\left(H_{3}\right) f(x, 0)=-x$.

Observamos que:

(1) Fixando as coordenadas $x_{1}=x_{2}=0$, de $\left(H_{1}\right)$ temos:

- $f_{1}(0, y)$ tem mesmo sinal que $y_{2}$, de modo que $f_{1}\left(0,0, y_{1}, 0\right)=0$;

- $f_{2}(0, y)$ tem mesmo sinal que $-y_{1}$, de modo que $f_{2}\left(0,0,0, y_{2}\right)=0$.

Portanto, $f(0,0)=0$ e além disso,

$$
\frac{\partial f_{1}(0)}{\partial y_{1}}=\frac{\partial f_{2}(0)}{\partial y_{2}}=0 \text {. }
$$

(2) $\mathrm{De}\left(H_{3}\right)$ vem que

$$
f_{1}(x, 0)=-x_{1} \text { e } f_{2}(x, 0)=-x_{2}
$$

Logo,

$$
\frac{\partial f_{1}(0)}{\partial x_{1}}=\frac{\partial f_{2}(0)}{\partial x_{2}}=-1 \text { e } \frac{\partial f_{1}(0)}{\partial x_{2}}=\frac{\partial f_{2}(0)}{\partial x_{1}}=0 \text {. }
$$

Consideraremos no restante deste cápítulo a equação (3.1) sujeita às hipóteses $\left(H_{1}\right),\left(H_{2}\right)$ e $\left(H_{3}\right)$.

\subsection{A equação característica}

Nosso propósito nesta seção é obter a equação característica de (3.1).

Seja

$$
B(\alpha)=\left(\begin{array}{cc}
0 & \delta_{1} \alpha \\
-\delta_{2} \alpha & 0
\end{array}\right)
$$


A linearização de (3.1) perto da origem é dada por

$$
\dot{x}(t)=(-\alpha I B(\alpha)) \operatorname{col}(x(t), x(t-1)),
$$

onde $I$ é a matriz identidade de ordem 2 .

Para que a equação (3.3) possua soluções não triviais da forma $x(t)=e^{\lambda t} u$, $u \in \mathbf{R}^{2}$, é necessário e suficiente que $u$ seja solução não nula de $\Delta(\lambda) u=0$, onde

$$
\Delta(\lambda):=(\lambda+\alpha) I-e^{-\lambda} B(\alpha)
$$

Se $u$ é solução não nula de $\Delta(\lambda) u=0$, então det $\Delta(\lambda)=0$, isto é,

$$
(\lambda+\alpha)^{2}+e^{-2 \lambda} \delta_{1} \delta_{2} \alpha^{2}=0 .
$$

Seja $\delta=\sqrt{\delta_{1} \delta_{2}}>0$. Chegamos então à seguinte equação característica de (3.3):

$$
(\lambda+\alpha)^{2} e^{2 \lambda}=-\delta^{2} \alpha^{2} .
$$

\subsection{Os auto valores da equação linearizada}

Partindo da análise de propriedades das raízes de (3.4), verificaremos a existência de soluções periódicas da equação (3.1) que emanam da solução nula, para certos valores do parâmetro $\alpha$.

Facilmente vemos que a única raiz real de (3.4) é $\lambda=0$ e ocorre somente para $\alpha=0$.

Como $\lambda=\lambda(\alpha)$ é raiz de (3.4) se, e somente se, seu complexo conjugado $\bar{\lambda}$ também for, podemos restringir nosso estudo ao semiplano superior $\Im \lambda>0$.

As raízes $\lambda$ de (3.4) que possuem parte imaginária positiva são distribuídas em faixas do tipo

$$
\begin{aligned}
& S_{0}:=\left\{\lambda \in \mathbf{C}: 0<\Im \lambda<\frac{\pi}{2}\right\} \\
& S_{k}:=\left\{\lambda \in \mathbf{C}:\left(k-\frac{1}{2}\right) \pi<\Im \lambda<\left(k+\frac{1}{2}\right) \pi\right\}
\end{aligned}
$$

$k=1,2, \ldots$. Isto é uma consequência do seguinte lema: 
Lema 3.1 Não existe raiz de (3.4) sobre as retas $b=\left(k+\frac{1}{2}\right) \pi, k \geq 0$.

\section{Demonstração:}

Se $\lambda=a+b i$, a equação (3.4) é equivalente ao sistema

$$
\left\{\begin{array}{l}
e^{2 a}\left[(a+\alpha)^{2}-b^{2}\right] \cos 2 b-2 b(a+\alpha) \operatorname{sen} 2 b=-\delta^{2} \alpha^{2} \\
{\left[(a+\alpha)^{2}-b^{2}\right] \operatorname{sen} 2 b+2 b(a+\alpha) \cos 2 b=0}
\end{array}\right.
$$

Tomando em particular $b=\left(k+\frac{1}{2}\right) \pi$, obtemos

$$
\left\{\begin{array}{l}
e^{2 a}\left[(a+\alpha)^{2}-b^{2}\right]=\delta^{2} \alpha^{2} \\
2 b(a+\alpha)=0
\end{array}\right.
$$

e esse sistema é incompatível.

Segue da equação (3.4) que $(\lambda+\alpha) e^{\lambda}$ é um número imaginário puro, dado por

$$
(\lambda+\alpha) e^{\lambda}= \pm i \delta|\alpha|
$$

Tomando $\lambda=a+b i$, obtemos o sistema

$$
\left\{\begin{array}{l}
(a+\alpha) \cos b-b \operatorname{sen} b=0 \\
(a+\alpha) \operatorname{sen} b+b \cos b= \pm \delta|\alpha| e^{-a}
\end{array}\right.
$$

Da primeira equação deste sistema tiramos que

$$
b(a+\alpha) \operatorname{sen} b \cos b=b^{2} \operatorname{sen}^{2} b \geq 0,
$$

ou seja, $(a+\alpha) \operatorname{sen} b$ e $b \cos b$ têm o mesmo sinal. Como em $S_{k}$ o sinal de $b \cos b$ é $(-1)^{k}$, segue da segunda equação do sistema que

$$
(a+\alpha) \operatorname{sen} b+b \cos b=(-1)^{k} \delta|\alpha| e^{-a} .
$$

Portanto, a equação (3.4) é equivalente a

$$
(\lambda+\alpha) e^{\lambda}=(-1)^{k} \delta|\alpha| i
$$

em cada faixa $S_{k}, k \geq 0$.

Lema 3.2 Cada faixa $S_{k}, k \geq 0$, contém exatamente uma raiz de (3.4) para cada $\alpha \neq 0$ e esta raiz é simples. 


\section{Demonstração:}

Consideraremos particularmente a faixa $S_{1}$ e mostraremos que se $\lambda \in S_{1}$ é raiz de (3.4), tanto $\Re \lambda$ quanto $\Im \lambda$ são funçōes do parâmetro $\alpha$, quer $\alpha$ seja positivo, ou negativo; consequentemente haverá apenas uma raiz $\lambda$ em $S_{1}$, para cada $\alpha \neq 0$.

Primeiramente verificaremos a asserção para valores positivos do parâmetro $\alpha$ e, em seguida, para $\alpha<0$.

- $\alpha>0$ :

Se $\lambda=a+b i \in S_{1}$, de (3.5) vem que

$$
\left\{\begin{array}{l}
a+\alpha=b \operatorname{tg} b \\
(a+\alpha) \operatorname{sen} b+b \cos b=-\delta \alpha e^{-a}
\end{array}\right.
$$

Substituindo a primeira equação de (3.6) na segunda, obtemos

$$
\delta \alpha e^{\alpha}=\frac{-b e^{b \operatorname{tg} b}}{\cos b}
$$

Definindo $\beta:=\delta \alpha e^{\alpha}$, sendo $\alpha>0$, segue que

$$
\frac{d \beta}{d \alpha}=\delta e^{\alpha}(1+\alpha)>0
$$

e, para $0<\alpha<\infty, \beta \in(0 . \infty)$.

Por outro lado, podemos expressar

$$
\beta=\Lambda(b):=\frac{-b e^{b \operatorname{tg} b}}{\cos b}
$$

e

$$
\frac{d \beta}{d b}=-e^{b \operatorname{tg} b} \sec b\left[(1+b \operatorname{tg} b)^{2}+b^{2}\right] .
$$

Como em $S_{1}$ o sinal de sec $b$ é negativo, temos $\frac{d \beta}{d b}>0$, ou seja, $\beta$ é função estritamente crescente de $b$.

Além disso,

$$
\lim _{b \rightarrow \frac{\pi}{2}^{+}} \Lambda(b)=0 \text { e } \lim _{b \rightarrow \frac{3 \pi^{-}}{2}} \Lambda(b)=+\infty
$$


Logo, $b$ é unicamente definido como função de $\beta$, para $\beta \in(0, \infty)$. Por sua vez, $\beta$ é uma função estritamente crescente de $\alpha$ e, portanto, $b=\Im \lambda$ é função estritamente crescente de $\alpha$, cujo domínio é $(0, \infty)$ e a imagem é $\left(\frac{\pi}{2}, \frac{3 \pi}{2}\right)$.

Sendo $b$ função de $\alpha$, vem da primeira equação de (3.6) que $a$ também o é.

Portanto, $\lambda=a+b i \in S_{1}$ é função de $\alpha$.

- $\alpha<0$ :

Para $\lambda=a+b i,(3.5)$ torna-se equivalente a

$$
\left\{\begin{array}{l}
a+\alpha=b \operatorname{tg} b \\
(a+\alpha) \operatorname{sen} b+b \cos b=\delta \alpha e^{-a}
\end{array}\right.
$$

Como anteriormente, definimos

$$
\tilde{\beta}=\delta \alpha e^{\alpha}=\frac{b e^{b \operatorname{tg} b}}{\cos b},
$$

$\frac{d \bar{\beta}}{d \alpha}=\delta e^{\alpha}(1+\alpha)$ é positiva para $\alpha \in(-1,0)$, negativa para $\alpha<-1$, e

$$
\frac{d \tilde{\beta}}{d b}=e^{b \operatorname{tg} b} \sec b\left[(1+b \operatorname{tg} b)^{2}+b^{2}\right]<0 .
$$

Portanto, $b=\Im \lambda$ é uma função estritamente crescente de $\alpha$ para $\alpha<-1$ e estritamente decrescente para $-1<\alpha<0$. Como anteriormente, $a=\Re \lambda$ também é função de $\alpha$ para $\alpha<0$.

Como $\Im \lambda \neq 0$ para $\alpha \neq 0$,

$$
\frac{d}{d \lambda}\left[(\lambda+\alpha) e^{\lambda}+\delta|\alpha| i\right]=e^{\lambda}(1+\lambda+\alpha) \neq 0
$$

e assim concluimos que $\lambda \in S_{1}$ é simples.

As demais faixas são tratadas de modo análogo, com ligeiras adaptações.

Observação 3.1: Outra informação que podemos tirar da demonstração do lema 3.2 é que a raiz característica $\lambda \in S_{k}$ é diferenciável com relação a $\alpha$, pois $\Re \lambda$ e $\Im \lambda$ são funções diferenciáveis de $\alpha$. Para $\alpha>0$, depois de algumas manipulações, chegamos a seguinte expressão para a derivada de $a=\Re \lambda$ com relação a $\alpha$ :

$$
\frac{d a}{d \alpha}=\frac{a(1+a+\alpha)+b^{2}}{\alpha\left[(1+a+\alpha)^{2}+b^{2}\right]}
$$


Então, se $a>0, \frac{d a}{d a}>0$ e, como também $\frac{d b}{d \alpha}>0, b=\Im \lambda$ é uma função estritamente crescente de $a$, em cada faixa $S_{k}$, para $\alpha>0$.

Lema 3.3 Para $\delta>1$ e $\alpha>0$, fica univocainiìnte determinada uma sequência $\left(\lambda_{k}\right)$ de raizes características tal que $\lambda_{k}=b_{k} i \in S_{k}$, sendo $b_{k}=\operatorname{arcsen}(-1)^{k} \frac{1}{6} e$ os valores $\alpha_{k}$ correspondentes dados por $\alpha_{k}=b_{k} / \sqrt{\delta^{2}-1}, k \geq 0$.

\section{Demonstraçào:}

Se $\lambda=b i$, de (3.5) obtemos

$$
\left\{\begin{array}{l}
\alpha=b \operatorname{tg} b \\
\alpha \operatorname{sen} h+b \cos b=(-1)^{k} \delta \alpha
\end{array}\right.
$$

Então,

$$
\operatorname{sen} b=(-1)^{k} \frac{1}{\delta} \text { e } \cos b=(-1)^{k} \frac{\sqrt{\delta^{2}-1}}{\delta}
$$

para todo $k \geq 0 \mathrm{e}$, portanto,

$$
\alpha=\frac{b}{\sqrt{\delta^{2}-1}}
$$

Assim, determinamos a sequência $\left(\lambda_{k}\right)$ de raízes características, onde $\lambda_{k}=b_{k} i$ $\operatorname{com}\left(k-\frac{1}{2}\right) \pi<b_{k}=\operatorname{arcsen}(-1)^{k} \frac{1}{6}<\left(k+\frac{1}{2}\right) \pi$.

Observamos pelo lema acima que $b_{k}=b_{0}+k \pi, k \geq 1$. Então, a sequência $\left(\alpha_{k}\right)$ dada acima é tal que $0<\alpha_{0}<\alpha_{1}<\ldots \rightarrow+\infty$, quando $k \rightarrow+\infty$.

Lema 3.4 Se $\delta>1$ e $\alpha>0$, as raízes $\lambda(\alpha)$ da equação (3.4), em cada faixa $S_{k}$, convergem para $\ln \delta+i\left(k+\frac{1}{2}\right) \pi$ quando $\alpha \rightarrow+\infty, k \geq 0$.

\section{Demonstrạ̧ão:}

Se $\lambda=a+b i \in S_{k}$, de (3.5) vem

$$
\left\{\begin{array}{l}
a+\alpha=b \operatorname{tg} b \\
e^{a} b \sec b=(-1)^{k} \delta \alpha
\end{array}\right.
$$

Multiplicando ambos os membros da segunda equação por sen $b$, obtemos

$$
e^{a}\left(1+\frac{a}{\alpha}\right)=(-1)^{k} \delta \operatorname{sen} b
$$


Como foi visto na demonstração do lema $3.2, b=\Im \lambda$ é uma função estritamente crescente de $\alpha>0$ com domínio $(0, \infty)$, imagem $\left(0, \frac{\pi}{2}\right)$ em $S_{0}$ e $\left(\left(k-\frac{i}{2}\right) \pi,\left(k+\frac{1}{2}\right) \pi\right)$ em $S_{k}, k \geq 1$.

Logo, $b \rightarrow\left(k+\frac{1}{2}\right) \pi$ quando $\alpha \rightarrow+\infty \mathrm{e}, \operatorname{assim}, \operatorname{sen} b \rightarrow(-1)^{k}$ quando $k \rightarrow+\infty$.

Portanto, $\lim _{\alpha \rightarrow+\infty}(-1)^{k} \delta \operatorname{sen} b=\delta$.

Para $\alpha$ suficientemente grande, $a$ é positivo, pois $e^{a}\left(1+\frac{a}{\alpha}\right) \rightarrow \delta>1$ quando $\alpha \rightarrow \infty$.

Segue deste fato que $a$ é limitada quando $\alpha \rightarrow+\infty$ e

$$
\delta=\lim _{\alpha \rightarrow \infty} e^{a}\left(1+\frac{a}{\alpha}\right)=\lim _{\alpha \rightarrow \infty} e^{a},
$$

ou seja,

$$
a=\Re \lambda \rightarrow \ln \delta \text { quando } \alpha \rightarrow \infty .
$$

Lema 3.5 Se $\lambda_{k}=a_{k}+b_{k} i \in S_{k}, k \geq 0$, são raizes de (3.4) para algum $\alpha>0$, então suas partes reais satisfazem $a_{0}>a_{1}>\ldots \rightarrow-\infty$.

\section{Demonstração:}

Através da mudança de variável $\mu=\lambda+\alpha$ e da reparametrização dada por $\beta=\delta \alpha e^{\alpha}$, a equação (3.4) torna-se equivalente a

$$
\mu^{2} e^{2 \mu}=-\beta^{2}
$$

A cada solução $\lambda=a+b i$ de (3.4) corresponde biunivocamente a solução $\mu=(a+\alpha)+b i \operatorname{de}(3.8)$.

Pelo lema 3.2, para cada $\beta>0$, cada faixa $S_{k}, k \geq 0$, contém uma única raiz de (3.8) e esta é simples.

O lema ficará demonstrado se verificarmos que a ordenação desejada ocorre para as raízes de (3.8)

Tomando $\mu=a+b i$ em norma na equação (3.8), vemos que as raízes $\mu_{k}, k \geq 0$, devem satisfazer

$$
\left(a^{2}+b^{2}\right) e^{2 a}=\beta^{2}
$$


Esta relação, para $\beta=\pi$, define $b$ como funsão de $a$ no intervalo $-\infty<a \leq 0$ e esta função é decrescente, pois

$$
\frac{d b}{d a}=-\frac{1}{b}\left(a+\pi^{2} e^{-2 a}\right)<0
$$

para $a \leq 0$.

Ainda, se $\beta=\pi$ e $\mu$ é raiz de (3.8), então $\mu$ é imaginária pura e, se $\mu \in S_{0}$, devemos ter $a>0$.

Logo, $a_{0}>a_{1}=0>\ldots>a_{k}>\ldots, k \geq 2$, e temos a ordenação especificada das partes reais das raízes com a restrição $\beta=\pi$.

Suponhamos que tal ordenação não ocorra para algurn valor do parâmetro $\beta$.

Como cada $a_{k}$ é uma função contínua de $\beta$, existirá algum $\beta_{0}$ para o qual a equação (3.8) tem raízes $\mu_{k}$ e $\mu_{l} \operatorname{com} a_{k}=a_{l}=a, k>l$.

Então, de (3.8) vem

$$
\left(a^{2}+b_{k}^{2}\right) e^{2 a}=\left(a^{2}+b_{l}^{2}\right) e^{2 a}
$$

e isto implica que $b_{k}=b_{l}$, que é uma contradição, pois $b_{k}>b_{l}$.

Portanto, devemos ter $a_{0}>a_{1}>\ldots>a_{k}>\ldots$

Se $a=a_{k}$ e $b=b_{k}$ em (3.9), vemos que $a_{k} \rightarrow-\infty$ quando $k \rightarrow \infty$.

Estes resültados nos permitem ter uma idéia do comportamento das raízes características no plano complexo, especialmente quando $\delta>1$ e $\alpha>0$.

Consideremos por um instante o caso em que $0 \leq \Im \lambda<\frac{\pi}{2}$. Sabemos que a origem 0 é raiz característica e, pelo lema $3.3, \lambda_{0}=\bar{i}$ arcsen $\frac{1}{\delta}$ também é. Com isso, e usando o fato que $b$ é uma função estritamente crescente de $a>0$, para $\alpha>0$, concluimos que $a$ deve ser negativo para $\alpha \in\left(0, \alpha_{0}\right)$.

Além disso, $\frac{d a}{d \alpha}=0$ para, no máximo, um valor positivo de $b$.

Lembramos que a localização das raízes de (3.4) apresenta uma simetria com relação ao eixo real.

Suponhamos $\delta>1$ e o parâmetro $\alpha$ percorrendo o semi eixo real positivo, partindo da origem. Quando $\alpha$ atinge o valor $\alpha_{0}$, um par de raízes conjugadas, $\lambda_{0}=\lambda\left(\alpha_{0}\right)$ e $\overline{\lambda_{0}}$, cruza pela primeira vez o eixo imaginário, da esquerda para direita, com "velocidade positiva". Surgem então, duas raízes conjugadas com 
parte real positiva. Quando $\alpha=\alpha_{1}$, um novo par $\lambda_{1}=\lambda\left(\alpha_{1}\right)$ e $\lambda_{1}$ cruza transversalmente o eixo imaginário e assim sucessivamente, sendo a transversalidade sempre garantida por $\Re \lambda^{\prime}\left(\alpha_{k}\right)>0$ para $k \geq 0$.

Tiramos então, as seguintes conclusões:

(i) Os auto valores $\lambda_{k}=\lambda_{k}\left(\alpha_{k}\right) \neq 0, k \geq 0$, são simples e para cada $k$ fixado, os auto valores $\lambda_{j} \neq \lambda_{k}, \bar{\lambda}_{k}$ satisfazem $\lambda_{j} \neq m \lambda_{k}$ para todo inteiro $m$.

(ii) $\frac{d}{d \alpha}\left[\Re\left(\lambda_{k}(\alpha)\right]_{\alpha=\alpha_{k}} \neq 0\right.$.

O Teorema da Bifurcação de Hopf nos conduz ao

Teorema 3.1 Suponhamos $\delta>1$. Então, existe uma sequência $\left(\alpha_{k}\right)$ de valores do parâmetro a para os quais a equação (3.1) tem uma bifurcação de Hopf em $\alpha=\alpha_{k}, k \geq 0$, e tal que $0<\alpha_{0}<\alpha_{1}<\ldots \rightarrow \infty$. O ramo que emana de $\alpha_{k}$ representa soluções $w$-periódicas, com $w$ próximo $a 2 \pi / b_{k}, b_{k}$ definido univocamente por

$$
b_{k}=\operatorname{arcsen}(-1)^{k} \frac{1}{\delta} \in\left(\left(k-\frac{1}{2}\right) \pi,\left(k+\frac{1}{2}\right) \pi\right) \cap(0, \infty), k \geq 0 .
$$

Para $\alpha<0$ é possível obter um resultado análogo ao enunciado no teorema 3.1 , tomando, contudo, $\delta$ de modo mais restrito.

Os lemas 3.1 e 3.2 contém informações para este caso e, vale também, a equivalência das equações (3.4) e (3.5).

O próximo lema corresponde ao lema 3.3 .

Lema 3.6 Para $\delta>1$ e $\alpha<0$, fica determinada de modo único uma sequência $\left(\lambda_{k}\right)$ de raizes caracteristicas tal que $\lambda=d_{k} i \in S_{k}, d_{k}=\operatorname{arcsen}(-1)^{k+1} \frac{1}{\delta}$ e os valores $\alpha_{k}$ correspondentes são dados por $\alpha_{k}=-d_{k} / \sqrt{\delta^{2}-1}, k \geq 1$.

\section{Demonstração:}

É análoga a do lema 3.3, considerando neste caso a equação

$$
(\lambda+\alpha) e^{\lambda}=(-1)^{k+1} \delta \alpha i,
$$

e observando que se $\lambda \in S_{0}$ é raiz de (3.4) com $\alpha<0$, devemos ter $\Re \lambda \neq 0$.

Segue do lema 3.6 que $d_{k}=d_{1}+(k-1) \pi$ para $k \geq 2, d_{1}=\operatorname{arcsen} \frac{1}{6} \in\left(\frac{\pi}{2}, \pi\right)$ e a correspondente sequência $\left(\alpha_{k}\right)$ tende a $-\infty$ quando $k \rightarrow+\infty$. 
Sendo

$$
\Re \lambda^{\prime}\left(\alpha_{k}\right)=-1-\frac{\left(\alpha_{k}+1\right)\left(\alpha_{k}+\alpha_{k}^{2}+d_{k}^{2}\right)}{\alpha_{k}\left[\left(1+\alpha_{k}\right)^{2}+d_{k}^{2}\right]},
$$

$\Re \lambda^{\prime}\left(\alpha_{k}\right) \neq 0$ se, e somente se,

$$
\left(\alpha_{k}+1\right)\left(\alpha_{k}+\alpha_{k}^{2}+d_{k}^{2}\right) \neq-\alpha_{k}\left[\left(1+\alpha_{k}\right)^{2}+d_{k}^{2}\right]
$$

ou seja,

$$
d_{k}^{2} \neq-\frac{2 \alpha_{k}\left(1+\alpha_{k}\right)^{2}}{1+2 \alpha_{k}}, k \geq 1
$$

Uma condição suficiente para que isto ocorra é que $\delta<\sqrt{\pi^{2}+1}$. Isso pode ser visto da seguinte forma:

Se $d_{k}^{2}$ for dado pelo quociente acima, então,

$$
\frac{2 \alpha_{k}\left(1+\alpha_{k}\right)^{2}}{1+2 \alpha_{k}} \leq 0
$$

Desde que $\alpha_{k}<0$, tiramos que $\alpha_{k}^{2}$ deve ser menor que 1/4. Pelo lema 3.6, $d_{k}^{2}=\alpha_{k}^{2}\left(\delta^{2}-1\right)$, e $\left(k-\frac{1}{2}\right) \pi<d_{k}<\left(k+\frac{1}{2}\right) \pi$. Disto é possivel concluir que $\delta>\sqrt{\pi^{2}+1}$. Consequentemente, $\delta<\sqrt{\pi^{2}+1}$ é uma condição suficiente para que valha (3.10).

Podemos então, enunciar um teorema análogo ao teorema 3.2, para o caso $\alpha<0$ :

Teorema 3.2 Seja $1<\delta<\sqrt{\pi^{2}+1}$. Existe uma sequência de valores de $\alpha$ satisfazendo $0>\alpha_{0}>\alpha_{1}>\ldots \rightarrow-\infty$, para os quais a equação (3.1) tem uma bifurcação de Hopf em $\alpha=\alpha_{k}, k \geq 1$. O ramo que emana de $\alpha_{k}$ representa soluções $w$-periódicas com $w$ próximo a $2 \pi / d_{k}, d_{k}$ definido univocamente por

$$
d_{k}=\operatorname{arcsen}(-1)^{k+1} \frac{1}{\delta} \in\left(\left(k-\frac{1}{2}\right) \pi,\left(k+\frac{1}{2}\right) \pi\right), k \geq 1 .
$$




\section{Apêndice A}

\section{Fórmula da Variação das Constantes}

Para cada $(\sigma, \phi) \in \mathbf{R} \times \mathcal{C}$, consideremos o sistema linear não homogêneo

$$
\left\{\begin{aligned}
\dot{x}(t) & =L\left(t, x_{t}\right)+h(t), \quad t \geq \sigma \\
x_{\sigma} & =\phi
\end{aligned}\right.
$$

onde $h \in \mathcal{L}_{1}^{\text {loc }}\left([\sigma, \infty), \mathbf{R}^{n}\right)$, o espaço das funções de $[\sigma, \infty)$ em $\mathbf{R}^{n}$ que são Lebesgue-integráveis em cada subconjunto compacto de $[\sigma, \infty)$. Assumiremos ainda que $L(t, \phi)$ é linear em $\phi$ e que existe uma função $\beta$ em $\mathcal{L}_{1}^{\text {loc }}(\mathbf{R}, \mathbf{R})$ tal que

$$
|L(t, \phi)| \leq \beta(t)|\phi|
$$

para todo $t \in \mathbf{R}$ e $\phi \in \mathcal{C}$.

Com estas hipóteses, segue da teoria geral das equaçóes lineares com retardamento, que para cada $(\sigma, \phi) \in \mathbf{R} \times \mathcal{C}$ e $h \in \mathcal{L}_{1}^{\text {loc }}\left([\sigma, \infty), \mathbf{R}^{n}\right)$, o sistema (A.1) possui uma única solução passando por $(\sigma, \phi)$, que é contínua em $[\sigma-r, \infty)$.

Seja então, $x(\sigma, \phi, h)$ a solução de (A.1) com valor inicial $\phi$ em $\sigma$. A linearidade de $L$ e a unicidade de soluções do sistema (A.1) implicam que

$$
x(\sigma, \phi, h)=x(\sigma, \phi, 0)+x(\sigma, 0, h)
$$

São válidas também as seguintes desigualdades:

$$
|x(\sigma, \phi, 0)(t)| \leq|\phi| e^{\int_{\sigma}^{t} \beta(\rho) d s}, t \geq \sigma
$$




$$
|x(\sigma, 0, h)(t)| \leq\left(\int_{\sigma}^{t}|h(s)| d s\right) e^{\int_{\sigma}^{t} \beta(s) d s}, t \geq \sigma .
$$

Segue destas relaçōes que $x(\sigma, ., 0)$ e $x(\sigma, 0,$.$) são funções lineares e contínuas$ em $\phi$ e $h$ respectivamente.

Estes fatos são apresentados em [5, cap.6].

Seja $U(t, s)$ a solução da equação

$$
\frac{\partial}{\partial t} U(t, s)=L\left(t, U_{t}(., s)\right), t \geq s, \text { q.s em } s \text { e } t
$$

que satisfaz

$$
U(t, s)= \begin{cases}0, & s-r \leq i<s \\ I, & t=s\end{cases}
$$

e $U_{t}(., s)(\theta)=U(t+\theta, s)$ para $-r \leq \theta \leq 0$.

A função $U(t, s)$ é chamada matriz fundamental.

Usando a continuidade de $x(\sigma, ., 0)$ e $x(\sigma, 0,$.$) , obteremos a fórmula da variação$ das constantes, que fornece uma representação da solução $x(\sigma, \phi, h)$ do sistema (A.1) em termos da matriz fundamental $U(t, s)$.

Precisaremos de um resultado de análise funcional sobre representação de operadores lineares contínuos em $\mathcal{L}_{1}\left([a, b], \mathbf{R}^{n}\right)$.

Lema A.1 Seja $T: \mathcal{L}_{1}\left([a, b], \mathbf{R}^{n}\right) \rightarrow \mathbf{R}^{n}$ um operador linear contínuo. Então existe uma função matricial $V(\theta), a \leq \theta \leq b$, integrável e essencialmente limitada, tal que

$$
T h=\int_{a}^{b} V(\theta) h(\theta) d \theta, h \in \mathcal{L}_{1}\left([a, b], \mathbf{R}^{n}\right)
$$

A matriz $V(\theta)$ é única, exceto para conjuntos de medida nula em $\theta$.

Teorema A.1 (Fórmula da Variação das Constantes) Sejam $L$ um operador linear satisfazendo as condições mencionadas acima, $h \in \mathcal{L}_{1}^{\text {loc }}\left([\sigma, \infty), \mathbf{R}^{n}\right)$, e $x(\sigma, \phi, h)$ a solução so sistema (A.1). Então,

$$
\begin{aligned}
x(\sigma, \phi, h)(t) & =x(\sigma, \phi, 0)(t)+\int_{\sigma}^{t} U(t, s) h(s) d s, t \geq \sigma \\
x_{\sigma} & =\phi,
\end{aligned}
$$

onde $U(t, s)$ é a matriz fundamental. 


\section{Demonstração:}

Como $x(\sigma, 0,):. \mathcal{L}_{1}\left([\sigma, t], \mathbf{R}^{n}\right) \rightarrow \mathbf{R}^{n}$ é uma função linear contínua, pelo lema A.1 existe uma matriz $n \times n, U^{*}(t, \sigma,.) \in \mathcal{L}_{\infty}\left([\sigma, t], \mathbf{R}^{n 2}\right), t \geq \sigma$, tal que

$$
x(\sigma, 0, h)(t)=\int_{\sigma}^{t} U^{*}(t, \sigma, s) h(s) d s, t \geq \sigma .
$$

Vamos mostrar que $U^{*}(t, \sigma, s)$ independe de $\sigma$. Sejam $\alpha \in[\sigma, t]$ e $k$ um elemento qualquer de $\mathcal{L}_{1}\left([\sigma, t], \mathbf{R}^{n}\right)$ que satisfaz $k(s)=0$ para $s \in[\sigma, \alpha]$. Então, como $x(\sigma, 0, k)(t)=0$ para $\sigma-r \leq t \leq \alpha$, segue que

$$
x(\sigma, 0, k)(t)=x(\alpha, 0, k)(t)
$$

para todo $t \geq \alpha$.

Isto implica que

$$
\int_{\alpha}^{t}\left[U^{*}(t, \sigma, s)-U^{*}(t, \alpha, s)\right] k(s) d s=0
$$

para todo $k \in \mathcal{L}_{1}\left([\alpha, t], \mathbf{R}^{n}\right)$.

Logo, $U^{*}(t, \sigma, s)=U^{*}(t, \alpha, s)$ q.s em $s$. Da arbitrariedade de $\alpha$, concluimos que $U^{*}(t, \sigma, s)$ independe de $\sigma$.

Definimos

$$
U(t, s):= \begin{cases}U^{*}(t, \sigma, s) & \text { para } s \leq t \\ 0 & \text { para } s-r \leq t<s\end{cases}
$$

Do sistema (A.1) segue que

$$
x(\sigma, 0, h)(t)=\int_{\sigma}^{t} L\left(s, x_{s}(\sigma, 0, h)\right) d s+\int_{\sigma}^{t} h(s) d s
$$

Pelo teorema da representação de Riesz, existe uma matriz função $\eta(t, \theta)$, mensurável em $(t, \theta)$, tal que

$$
L(t, \phi)=\int_{-\tau}^{0}\left[d_{\theta} \eta(t, \theta)\right] \phi(\theta)
$$

então, de (A.9) obtemos

$$
\int_{\sigma}^{t} U(t, s) h(s) d s=\int_{\sigma}^{t}\left(\int_{-\tau}^{0}\left[d_{\theta} \eta(s, \theta)\right] \int_{\sigma}^{\rho+\theta} U(s+\theta, u) h(u) d u\right) d s+\int_{\sigma}^{t} h(s) d s
$$




$$
\begin{aligned}
& =\int_{0}^{t}\left(\int_{-r}^{0}\left[d_{\theta} \eta(s, \theta)\right] \int_{\sigma}^{\theta} U(s+\theta, u) h(u) d u\right) d s+\int_{\sigma}^{t} h(s) d s \\
& =\int_{0}^{t}\left[\int_{u}^{t}\left(\int_{-r}^{0}\left[d_{\theta} \eta(s, \theta) U(s+\theta, u)\right) d s\right] h(u) d u+\int_{\sigma}^{t} h(s) d s\right. \\
& =\int_{\sigma}^{t}\left[\int_{\theta}^{t}\left(\int_{-r}^{0} d_{\theta} \eta(u, \theta) U(u+\theta, s)\right) d u\right] h(s) d s+\int_{\sigma}^{t} h(s) d s
\end{aligned}
$$

para todo $h \in \mathcal{L}_{1}\left([\sigma, t], \mathbf{R}^{n}\right)$.

Esta relação sügere que

$$
U(t, s)=\int_{0}^{t}\left(\int_{-r}^{0}\left[d_{\theta} \eta(u, \theta)\right] U(u+\theta, s)\right) d u+I \text { q.s em } s .
$$

Podemos então, tomar esta relação como definição de $U(t, s)$ para $t \geq s \mathrm{com}$ $U(t, s)=0$ para $t<s$. Para cada $s$ fixado, existe uma única solução desta equação que é contínua para $t \geq s$. Além disso, $U(t, s)$ é mensurável em $t$ e $s$. Temos, portanto, condições suficientes de regularidade, que tornam viáveis as manipulações feitas acima.

Isto mostra que $U(t, s)$ na fórmula da variação das constantes realmente satisfaz esta equação integral.

Derivando $U(t, s)$ com relação a $t$ obtemos as relações (A.6) e (A.7); isto completa a prova do teorema.

Para sistemas autônomos segue trivialmente o seguinte corolário:

Corolário A.1 Se o sistema (A.1) é autônomo, então

$$
U(t, s)=U(t-s, 0):=U(t-s)
$$

$e$

$$
\begin{aligned}
x(\sigma, \phi, h)(t) & =x(t-\sigma, \phi, 0)(0)+\int_{\sigma}^{t} U(t-s) h(s) d s, t \geq \sigma \\
x_{\sigma} & =\phi
\end{aligned}
$$

Às vezes é conveniente ter a fórmula da variação das constantes expressa de um modo diferente. Se são válidas as hipóteses do teorema A.1, então temos

$$
\begin{aligned}
& x(\sigma, \phi, h)(t+\theta)=x(\sigma, \phi, o)(t+\theta)+\int_{0}^{t+\theta} U(t+\theta, s) h(s) d s, t+\theta \geq \sigma \\
& x(\sigma, \phi, h)(t+\theta)=\phi(t+\theta), \quad \sigma-r \leq t+\theta \leq \sigma,-r \leq \theta \leq 0 .
\end{aligned}
$$

68 
Como $U(t+\theta, s)=0$ para $s>t+\theta$, e $x(\sigma, \phi, 0)(t+\theta)=\phi(t-\sigma+\theta)$ para $\sigma-r \leq t+\theta \leq \sigma$, podemos combinar estas duas expressóes numa única, dada por

$$
x(\sigma, \phi, h)(t+\theta)=x(\sigma, \phi, 0)(t+\theta)+\int_{\sigma}^{t} U(t+\theta, s) h(s) d s, t \geq \sigma,-r \leq \theta \leq 0,
$$

ou ainda,

$$
x_{t}(\sigma, \phi, h)=x_{t}(\sigma, \phi, 0)+\int_{0}^{t} U_{t}(., s) h(s) d s, t \geq \sigma,
$$

sendo esta equação integral entendida agora como integral no espaço euclidiano; todas operações usuais das integrais são válidas para (A.11).

A aplicação solução associada à eq̣uação $\dot{x}(t)=L\left(t, x_{t}\right)$, dada por

$$
T(t, \sigma) \phi=x_{t}(\sigma, \phi, 0)
$$

é linear e contínua. Considerando que as relações (A.6) e (A.7) estão satisfeitas em $t$ para todo $t>s+r$ e quase sempre em $s$, podemos escrever

$$
U_{t}(., s)=T(t, s) X_{0}
$$

onde

$$
X_{0}(\theta)= \begin{cases}0 & -r \leq \theta<0 \\ I & \theta=0\end{cases}
$$

Com essa notação, a equação (A.11) torna-se

$$
x_{t}(\sigma, \phi, h)=T(t, \sigma) \phi+\int_{\sigma}^{t} T(t, s) X_{0} h(s) d s, t \geq \sigma .
$$

Essa última fórmula é mais usual do que (A.9). 


\section{Apêndice B}

\section{Método de Liapunov - Schmidt}

Muitos problemas em análise podem ser reduzidos a determinação dos zeros de uma função iuum espaço de Banach.

Para uma classe particular de funçōes, existe um método clássico frequentemente utilizado para investigação das soluções, referido como Método de Liapunov - Schmidt.

Daremos a seguir uma descrição deste método.

Sejam $X, Y, \Lambda$ espaços de Banach, $A: \mathcal{D}(A) \subset X \rightarrow Y$ um operador linear contínuo e $N: X \times \Lambda \rightarrow Y$ um operador contínuo juntamente com sua derivada de Frechét em $X$, tal que

$$
N(0,0)=0, \quad \frac{d}{d x} N(0,0)=0
$$

O problema consiste em determinar soluçães da equação

$$
A x-N(x, \lambda)=0
$$

para $(x, \lambda)$ numa vizinhança de $(0,0)$.

Suponhamos válida a seguinte hipótese:

(H) Existem projeções $P: X \rightarrow X, Q: Y \rightarrow Y$ tais que

$$
P(X)=\mathcal{N}(A) \text { e }(I-Q)(Y)=\mathcal{R}(A)
$$


onde $I$ denota o operador identidade.

Lembramos que uma projeção $p$ definida num espaço de Banach $X$, é um operador linear contínuo de $X$ em $X$, que satisfaz $p^{2}=p$.

A equação (B.1) é equivalente ao sistema

$$
\left\{\begin{array}{l}
(I-Q)[A x-N(x, \lambda)]=0 \\
Q[A x-N(x, \lambda)]=0
\end{array}\right.
$$

Como $A x=(I-Q) Y$ e $Q$ é projeçăo, para todo $x \in \mathcal{D}(A)$ temos

$$
(I-Q) A x=A x \text { e } Q A x=0
$$

Portanto, (B.2) é equivalente a

$$
\left\{\begin{array}{l}
A x=(I-Q) N(x, \lambda) \\
Q N(x, \lambda)=0
\end{array}\right.
$$

Cada $x \in \mathcal{D}(A)$ pode ser decomposto como $x=x_{1}+x_{2}$, com $x_{1} \in \mathcal{D}(A) \cap P(X)$ e $x_{2} \in \mathcal{D}(A) \cap(I-P)(X)$. Então, $A x=A x_{2}$ e a imagem de $\mathcal{D}(A) \cap(I-P)(X)$ por $A$ é $\mathcal{R}(A)$.

O operador $\left.A\right|_{\mathcal{D}(A) \cap(I-P)(X)}$ é um isomorfismo algébrico entre $\mathcal{D}(A) \cap(I-P)(X)$ e $\mathcal{R}(A)$.

Isso implica na existência de um operador linear $K: \mathcal{R}(A) \rightarrow(I-P)(X)$, que é limitado e satisfaz

$$
\begin{array}{ll}
A K=I & \text { em } \mathcal{R}(A) \mathrm{e} \\
K A=I-P & \text { em } \mathcal{D}(A)
\end{array}
$$

$K$ é chamado inversa à direita de $A$.

Estas relações garantem que (B.3) é equivalente a

$$
\begin{gathered}
x-P x=K(I-Q) N(x, \lambda) \\
Q N(x, \lambda)=0
\end{gathered}
$$

As equações (B.4) e (B.5) recebem respectivamente os nomes de equação auxiliar e equação de bifurcação. 
Se $y=x-a \operatorname{com} a=P x$, a equação (B.4) pode ser reescrita como

$$
y=K(I-Q) N(a+y, \lambda)
$$

Então, a função $J:(I-P)(X) \times \mathcal{N}(A) \times \Lambda \rightarrow(I-p)(X)$ dada por

$$
J(y, a, \lambda)=y-K(I-Q) N(y+a, \lambda)
$$

satisfaz

(i) $J(0,0,0)=0$

(ii) $\frac{d}{d y} J(0,0,0)$ é a identidade de $(I-P)(X)$.

Aplicando o teorema da função implícita à equação $J(y, a, \lambda)=0$, obtemos uma única solução $y^{*}=y^{*}(a, \lambda)$ definida numa vizinhança de $(y, a, \lambda)=(0,0,0)$.

Logo, o problema de encontrar soluções para a equação (B.1) se reduz ao de encontrar soluções da equação

$$
Q N\left(a+y^{*}(a, \lambda), \lambda\right)=0
$$

Obter uma solução.de (B.1) equivale a resolver (B.7) em relação $a(a, \lambda)$, onde $a$ é um elemento de $\mathcal{N}(A)$. Geralmente, este último problema está num espaço de dimensão menor que a dimensão de $X$, uma vez que $\mathcal{N}(A) \subseteq X$.

Um caso particularmente interessante se dá quando $A$ é um operador de Fredholm, isto é, as dimensões de $\mathcal{N}(A)$ e $\mathcal{R}(A)$ são finitas. Quando isto ocorre, o problema original se reduz à investigação de soluções de um sistema de $n$ equaçôes com $m$ incógnitas, onde $n=\operatorname{codim} \mathcal{R}(A)$ e $m=\operatorname{dim} \mathcal{N}(A)$.

Este método é amplamente usado na teoria de bifurcação. 


\section{Bibliografia}

[1] BAPTISTINI, M.T.Z. Soluções periódicas de uma equação diferencial no plano com retardamento global. Tese de Doutorado apresentada ao ICMSCUSP, 1990.

[2] CHOW, S.N.; HALE, J.K. Methods of bifurcation theory. New York, Springer, 1982.

[3] HALE, J.K. Functional differential equations New York, Springer, 1971. (Applied Mathematics Sciences, 3).

[4] HALE, J.K. Ordinary differential equations. New York, Krieger, 1980.

[5] HALE, J.K. Theory of functional differential equations. New York, Springer, 1977.

[6] HILLE, E.; PHILLIPS, R. Functional analysis and semigroups. Providence. AMS, 1957. (Colloquium Publications, 31).

[7] HONIG, C.S. Aplicações da topologia à análise. Rio de Janeiro, IMPA (Projeto Euclides), 1976.

[8] LEVINGER, B.W. A folk theorem in functional differential equations. JOURNAL OF DIFFERENTIAL EQUATIONS, v.4, p.612-9. 1968.

[9] LUNEL, S.M.V. Exponential type calculus for linear delay equations. Amsterdam, Centrum voor en Informatica, 1988.

[10] MARSDEN, J.E.; MCCRACKEN, M. The Hopf bifurcation and its applications. New York, Springer, 1976. (Applied Mathematical Science, 19).

[11] RUDIN, W. Functional analysis. New York, McGraw-Hill, 1973.

[12] STACKGOLD, I. Branching of solutions of nonlinear equations. SIAM REVIEW, v.13, n.3, p.289-332, 1971. 
[13] TÁBOAS, P.Z. Periodic solutions of a planar delay equation. PROC. ROYAL SOCIETY EDINBURGH, v.116A, p.85-101, 1990.

[14] TAYLOR, A.L. Introduction to functional analysis. New York, Wiley, 1958. 